\title{
Keys, Nominals, and Concrete Domains
}

Carsten Lutz

Theoretical Computer Science, TU Dresden

D-01062 Dresden, Germany

Carlos Areces

INRIA Lorraine, Nancy

54602 Villers les Nancy Cedex, France

Ian Horrocks

Department of Computer Science

University of Manchester

Oxford Road, Manchester M13 9PL, UK

Ulrike Sattler

Department of Computer Science

University of Manchester

Oxford Road, Manchester M13 9PL, UK
LUTZ@TCS.INF.TU-DRESDEN.DE

ARECES@LORIA.FR

HORROCKS@CS.MAN.AC.UK

SATTLER@CS.MAN.AC.UK

\begin{abstract}
Many description logics (DLs) combine knowledge representation on an abstract, logical level with an interface to "concrete" domains like numbers and strings with built-in predicates such as $<,+$, and prefix-of. These hybrid DLs have turned out to be useful in several application areas, such as reasoning about conceptual database models. We propose to further extend such DLs with key constraints that allow the expression of statements like "US citizens are uniquely identified by their social security number". Based on this idea, we introduce a number of natural description logics and perform a detailed analysis of their decidability and computational complexity. It turns out that naive extensions with key constraints easily lead to undecidability, whereas more careful extensions yield NEXPTIME-complete DLs for a variety of useful concrete domains.
\end{abstract}

\section{Motivation}

Description logics (DLs) are a family of formalisms that allow the representation of and reasoning about conceptual knowledge in a structured and semantically well-understood manner (Baader, Calvanese, McGuinness, Nardi, \& Patel-Schneider, 2003). The central entities for representing such knowledge are concepts, which are constructed from atomic concept names (unary predicates) and role names (binary relations) by means of the concept and role constructors offered by a particular DL. For example, in the basic propositionally closed description logic $\mathcal{A L C}$ (Schmidt-Schauß \& Smolka, 1991), we can describe a company that has part-time employees but only full-time managers using the concept

$$
\text { Company } \sqcap \text { ヨemployee.Parttime } \sqcap \forall \text { employee.( } \neg \text { Manager } \sqcup \neg \text { Parttime). }
$$

In this example, all words beginning with uppercase letters denote concept names while employee denotes a role name. 
Rather than being viewed only as conceptual entities in a knowledge base, concepts can, more generally, be understood as the central notion in various kinds of class-centered formalisms. In the last decade, this observation has given rise to various new and challenging applications of description logics such as reasoning about database conceptual models expressed in entity-relationship diagrams or object-oriented schemas (Calvanese, Lenzerini, \& Nardi, 1998; Calvanese, De Giacomo, \& Lenzerini, 1998) and reasoning about ontologies for use in the semantic web (Baader, Horrocks, \& Sattler, 2002a; Horrocks, 2002; Horrocks, Patel-Schneider, \& van Harmelen, 2002). These new applications have, in turn, stimulated research in description logics since the expressive power of existing DLs was insufficient for the new tasks. One important extension is by providing expressive means that allow the integration of numbers and other datatypes: suppose, for example, that we want to extend our earlier descriptions of companies and employees to include the founding year of a company and the hiring year of an employee. Then, we may want to describe companies that were founded before 1970 and state that the hiring year of employees is not prior to the founding year of the employing company. To do this, we obviously need a way to talk about natural numbers (such as 1970) and comparisons between natural numbers.

Nowadays, the standard approach to integrate datatypes into description logics is to extend DLs with concrete domains, as first proposed by Baader and Hanschke (1991a) and recently surveyed by Lutz (2003). More precisely, a concrete domain $\mathcal{D}$ consists of a set (such as the natural numbers) and predicates which are associated with a fixed extension over this set ${ }^{1}$ (such as the unary $=_{0}$, the binary $<$, and the ternary + ). The integration of concrete domains into, say, the description logic $\mathcal{A L C}$ is achieved by adding

1. abstract features, i.e. functional roles;

2. concrete features, i.e. (partial) functions associating values from the concrete domain (e.g., natural numbers) with logical objects;

3. a concrete domain-based concept constructor.

The DL obtained by extending $\mathcal{A L C}$ in this way is called $\mathcal{A L C}(\mathcal{D})$, where $\mathcal{D}$ denotes a concrete domain that can be viewed as a parameter to the logic. For example, using a suitable concrete domain $\mathcal{D}$ we can describe the constraints formulated above: the concept

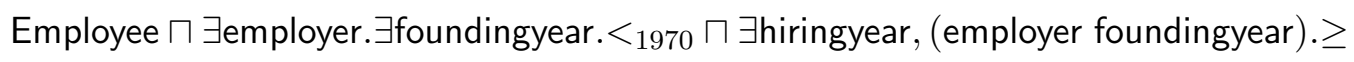

describes the set of employees who are employed by a company founded before 1970 and who have a hiring year not prior to the company's founding year. In this example, the term " $\exists$ foundingyear. $<1970$ " is an instance of the concrete domain concept constructor (not to be confused with the existential value restriction as in Eemployee.Parttime), and so is the third conjunct. While $<_{1970}$ is a unary predicate and thus the former instance only takes one concrete feature foundingyear as argument, the second instance uses the binary predicate " $\geq$ " requiring two arguments: the concrete feature hiringyear and the sequence of features (employer foundingyear) consisting of the abstract feature employer and the concrete feature foundingyear.

Concrete domains are rather important in many applications of DLs, including the two mentioned above:

1. This fixed extension is why these predicates are often called "built-in". 
- The standard way of using description logics for reasoning about conceptual database models is to translate a given model into a DL representation and then use a DL reasoner such as FaCT (Horrocks, 1998) or RACER (Haarslev \& Möller, 2001) to compute the consequences of the information provided explicitly in the model. This includes detecting inconsistencies and inferring additional, implicit containments between entities/classes (Calvanese et al., 1998). Since most databases store "concrete" data like numbers and strings, constraints concerning such data are usually part of the conceptual model and should thus also be captured by the description logic used for reasoning. Indeed, the above example concepts can be viewed as the DL encoding of constraints from a database about companies and their employees. As discussed by Lutz (2002c), description logics with concrete domains are well-suited for conceptual modeling applications involving concrete datatypes.

- So-called concrete datatypes play a prominent role in the construction of ontologies (Horrocks et al., 2002). Say, for example, that we want to construct an ontology which can be used for describing car dealers' web pages and web services. In such an ontology, concrete datatypes such as prices, manufacturing years, and names of car models will doubtlessly be very important. To formulate this ontology using a DL, we need a way to represent these concrete datatypes. Consequently, almost all DLs that have been proposed as an ontology language are equipped with some form of concrete domain (Fensel, van Harmelen, Horrocks, McGuinness, \& Patel-Schneider, 2001; Horrocks et al., 2002; Dean, Connolly, van Harmelen, Hendler, Horrocks, McGuinness, Patel-Schneider, \& Stein, 2002). Furthermore, since these ontology languages provide inverse abstract roles and functional restrictions, the users and ontology designers were quite surprised to find that they do not provide inverse concrete functional features - which is due to the fact that such features correspond to "concrete key constraints", for which no reasoning algorithms were known and whose effect on the decidability/complexity was not yet investigated.

In this paper, we propose to further enhance the expressive power of description logics with concrete domains by extending them with "concrete key constraints". This extension is useful both for knowledge representation and for the two applications sketched above. The following three examples describe the basic idea.

1. Suppose that, in a knowledge representation application, we represent nationalities by concept names such as US and German and, for US citizens, we store the social security number using a concrete feature ssn. Then it would be natural to state that US citizens are uniquely identified by their social security number, i.e. any two distinct instances of

$$
\text { Human } \sqcap \exists \text { nationality.US }
$$

must have different values for the ssn feature. In our extension of DLs with concrete domains, this can be expressed by using the key assertion ${ }^{2}$

(ssn keyfor Human $\sqcap \exists$ nationality.US).

2. Readers familiar with the relationship between DLs and first order logic will notice that this key assertion is equivalent to $\forall x_{1} x_{2} \cdot\left(\left(\bigwedge_{i \in\{1,2\}}\left(\operatorname{Human}\left(x_{i}\right) \wedge \exists z \cdot\left(\right.\right.\right.\right.$ nationality $\left.\left.\left.\left(x_{i}, z\right) \wedge \mathrm{US}(z)\right)\right) \wedge \neg\left(x_{1}=x_{2}\right)\right) \Rightarrow \neg\left(\operatorname{ssn}\left(x_{1}\right)=\right.$ $\left.\left.\operatorname{ssn}\left(x_{2}\right)\right)\right)$. 
2. Returning to our database about companies and employees, it could be useful to equip every employee with (i) a concrete feature branch storing the branch-ID in which she is working and (ii) a concrete feature id storing her personnel-ID. It would then be natural to enforce that the branch-ID together with the personnel-ID uniquely identifies employees, even though personnel-IDs are not unique. We can do this by using the composite key assertion

(branch, id keyfor Employee).

3. In the car dealers' ontology, we may assume that cars as well as manufacturers are equipped with identification numbers and that every car is uniquely identified by the combination of its own identification number and its manufacturers one. To express this, we could employ a composite key assertion referring to sequences of features, in this case (manufacturer id):

$$
\text { (id, (manufacturer id) keyfor Car). }
$$

More formally, we propose to extend DLs to provide for concrete domains with key boxes, which are sets of key assertions of the form

$$
\left(u_{1}, \ldots, u_{n} \text { keyfor } C\right) \text {, }
$$

where each $u_{i}$ is a sequence $f_{1} \cdots f_{n} g$ of abstract features $f_{j}$ followed by a single concrete feature $g$, and $C$ is a concept. As the above examples illustrate, the idea of key constraints is very natural. Since, moreover, keys play an important role in databases and, as mentioned above, reasoning about database conceptual models is an important, challenging application of description logics, several approaches to extend description logics with keys have already been investigated (Borgida \& Weddell, 1997; Calvanese, De Giacomo, \& Lenzerini, 2000; Khizder, Toman, \& Weddell, 2001). What distinguishes our approach from existing ones, however, is the idea of using concrete domains for constructing key constraints, rather than defining keys on an abstract, logical level.

The goal of this paper is to provide a comprehensive analysis of the effects on decidability and computational complexity of adding key boxes to description logics with concrete domains. To this end, we extend the two description logics $\mathcal{A L C}(\mathcal{D})$ and $\mathcal{S H O} \mathcal{Q}(\mathcal{D})$ with key boxes, in this way obtaining $\mathcal{A L C K}(\mathcal{D})$ and $\mathcal{S H O} \mathcal{Q K}(\mathcal{D})$, respectively. While the basic DL with concrete domains $\mathcal{A L C}(\mathcal{D})$ has already been discussed above, $\mathcal{S H O} \mathcal{Q}(\mathcal{D})$ was proposed as an ontology language in (Horrocks \& Sattler, 2001). It provides a wealth of expressive possibilities such as general concept inclusion axioms (GCIs), transitive roles, role hierarchies, nominals, and qualifying number restrictions. Moreover, it offers a restricted variant of the concrete domain constructor that disallows the use of sequences of features in order to avoid undecidability of reasoning. The main outcome of our investigations is that key constraints can have a dramatic impact on the decidability and complexity of reasoning: for example, whereas satisfiability of $\mathcal{A L C}(\mathcal{D})$-concepts is known to be PSPACE-complete (Lutz, 2002 b), we will show that satisfiability of $\mathcal{A L C K}(\mathcal{D})$-concepts w.r.t. key boxes is, in general, undecidable. Decidability can be regained if we restrict the concepts used in key boxes 
to Boolean combinations of concept names (Boolean key boxes). Interestingly, satisfiability of $\mathcal{A L C K}(\mathcal{D})$-concepts w.r.t. Boolean key boxes is still NExpTIME-complete even for very simple concrete domains. In the case of $\mathcal{S H O Q}(\mathcal{D})$ and $\mathcal{S H O} \mathcal{Q K}(\mathcal{D})$, the leap in complexity is somewhat less dramatic since $\mathcal{S H O Q}(\mathcal{D})$-concept satisfiability is already ExPTIME-complete: again, the addition of key boxes results in NExPTIME-complete reasoning problems.

It is interesting to note that there exists a close connection between key assertions and so-called nominals, i.e. concept names that can have at most one instance, such as Pope. Nominals are a standard means of expressivity in description logics and sometimes appear in disguise as the "one-of" operator (Borgida \& Patel-Schneider, 1994; Horrocks et al., 2002). It is not hard to see that key boxes can "simulate" nominals: if, for example, we use a concrete domain based on the natural numbers and providing unary predicates $={ }_{n}$ for equality with $n \in \mathbb{N}$, then the key assertion ( $g$ keyfor $\top$ ), where $T$ stands for logical truth, obviously makes the concept $\exists g .={ }_{n}$ behave like a nominal, for each $n \in$ $\mathbb{N}$. For this reason, we also consider $\mathcal{A L C O}(\mathcal{D})$, the extension of $\mathcal{A L C}(\mathcal{D})$ with nominals, and $\mathcal{A L C O K}(\mathcal{D})$, the extension of $\mathcal{A L C K}(\mathcal{D})$ with nominals. ${ }^{3}$ Our main result concerning nominals is that, although in general being of lower expressive power than key boxes, they already make reasoning NExPTIME-hard if combined with concrete domains: there exist concrete domains $\mathcal{D}$ such that $\mathcal{A} \mathcal{L C O}(\mathcal{D})$-concept satisfiability is NExPTIME-complete. We should like to stress that this and the other NExPTime-hardness results obtained in this paper are in accordance with the observation made in (Lutz, 2004) that the PSPACE-upper bound for reasoning with $\mathcal{A L C}(\mathcal{D})$ is not robust w.r.t. extensions of the logic: there exist several "seemingly harmless" extensions of $\mathcal{A L C}(\mathcal{D})$ (for example with acyclic TBoxes and with inverse roles) which make the complexity of reasoning leap from PSPACE-completeness to NExPTime-completeness for many natural concrete domains.

The remainder of this paper is organized as follows: in Section 2, we formally introduce concrete domains, key boxes, and the $\mathrm{DL} \mathcal{A} \mathcal{L C O} \mathcal{K}(\mathcal{D})$ together with its fragments $\mathcal{A L C K}(\mathcal{D})$ and $\mathcal{A L C O}(\mathcal{D})$. Moreover, we define Boolean key boxes, which only allow Boolean combinations of concept names to appear in key definitions. Additionally, we introduce some other important properties of key boxes: path-free key boxes prohibit the use of sequences of features in key assertions; in unary key boxes, each key assertion involves exactly one sequence of features; and composite key boxes are simply non-unary ones.

Section 3 is devoted to establishing lower bounds for extensions of $\mathcal{A L C}(\mathcal{D})$ with key boxes or nominals. In Section 3.1, we use a reduction of the Post Correspondence Problem to prove that $\mathcal{A L C K}(\mathcal{D})$-concept satisfiability w.r.t. (non-Boolean) key boxes is undecidable for a large class of concrete domains. We then shift our attention to Boolean key boxes since, in Section 4, we show that this restriction restores decidability. In Section 3.2, we introduce a NExPTIME-complete variant of the domino problem and three concrete domains that are useful for the reduction of this problem to concept satisfiability in DLs with Boolean key boxes or nominals. In Section 3.3, we use these concrete domains to prove that $\mathcal{A} \mathcal{L C K}(\mathcal{D})$-concept satisfiability w.r.t. Boolean, path-free and unary key boxes is NExPTime-hard for some natural concrete domains. In Section 3.4, we prove that there exist concrete domains $\mathcal{D}$ such that $\mathcal{A L C O}(\mathcal{D})$-concept satisfiability without reference to

3. Note that the logic $\mathcal{S H O} \mathcal{Q}(\mathcal{D})$ already provides for nominals. 
key boxes is already NExPTIME-hard; we show that this is true even for some concrete domains that are computationally very simple (PTIME) when considered in isolation.

The purpose of Section 4 is to develop reasoning procedures for description logics with key boxes and to prove upper complexity bounds matching the NExPTIME lower bounds established in the previous section. We start in Section 4.1 with a tableau algorithm that decides $\mathcal{A L C O K}(\mathcal{D})$-concept satisfiability w.r.t. Boolean key boxes, provided that the concrete domain $\mathcal{D}$ is key-admissible. Intuitively, a concrete domain $\mathcal{D}$ is key-admissible if there exists an algorithm that takes a finite conjunction $c$ of predicates from $\mathcal{D}$ over some set of variables, decides whether this conjunction is satisfiable and, if so, chooses a solution of $c$ and returns the information on which variables take the same values in it. We call such an algorithm a $\mathcal{D}$-tester. We have chosen a tableau algorithm since this type of reasoning procedure has the potential to be implemented in efficient reasoners and has been shown to behave well in practice (Horrocks, Sattler, \& Tobies, 2000; Haarslev \& Möller, 2001). Our algorithm implies the following upper complexity bound: if $\mathcal{D}$ is a key-admissible concrete domain for which a non-deterministic polynomial time $\mathcal{D}$-tester exists, then $\mathcal{A L C O}(\mathcal{D})$ concept satisfiability w.r.t. Boolean key boxes is in NExPTIME.

In Section 4.2, we devise a tableau algorithm for $\mathcal{S H O Q \mathcal { K }}(\mathcal{D})$-concept satisfiability w.r.t. path-free key boxes which might involve non-Boolean concepts. For the decidability of $\mathcal{A L C O K}(\mathcal{D})$, we restricted key boxes to Boolean ones. For $\mathcal{S H O} \mathcal{O K}(\mathcal{D})$, such a restriction is not possible since $\mathcal{S H O} \mathcal{Q}(\mathcal{D})$ provides TBoxes, and we can thus no longer distinguish between Boolean and non-Boolean concepts. On the other hand, it follows from an undecidability proof by Baader and Hanschke $(1992)$ that $\mathcal{S H O} \mathcal{Q}(\mathcal{D})$ is undecidable if we allow for sequences of features in concrete domain constructors. Thus we restrict key assertions analogously to path-free ones, and show that this yields indeed a decidable logic. Its expressive power is orthogonal to the one of $\mathcal{A L C O K}(\mathcal{D})$, and our previous undecidability results imply that the combination of $\mathcal{A L C O K}(\mathcal{D})$ and $\mathcal{S H O} \mathcal{Q K}(\mathcal{D})$ is undecidable. As a by-product of the correctness proof of the algorithm, we obtain a bounded model property for $\mathcal{S H O} \mathcal{Q} \mathcal{K}(\mathcal{D})$, which implies that $\mathcal{S H O} \mathcal{H} \mathcal{K}(\mathcal{D})$-concept satisfiability w.r.t. path-free key boxes is in NExPTIme if $\mathcal{D}$ is a key-admissible concrete domain for which a nondeterministic polynomial time $\mathcal{D}$-tester exists.

In Section 5, we summarize the results obtained and give an outlook to possible future research.

\section{Description Logics with Concrete Domains}

In the following, we introduce the description logic $\mathcal{A} \mathcal{L C O K}(\mathcal{D})$. Let us start by defining concrete domains:

Definition 2.1 (Concrete Domain). A concrete domain $\mathcal{D}$ is a pair $\left(\Delta_{\mathcal{D}}, \Phi_{\mathcal{D}}\right)$, where $\Delta_{\mathcal{D}}$ is a set and $\Phi_{\mathcal{D}}$ a set of predicate names. Each predicate name $P \in \Phi_{\mathcal{D}}$ is associated with an arity $n$ and an $n$-ary predicate $P^{\mathcal{D}} \subseteq \Delta_{\mathcal{D}}^{n}$.

Based on concrete domains, we can now define $\mathcal{A L C O K}(\mathcal{D})$-concepts and key boxes.

Definition 2.2 $(\mathcal{A L C O K}(\mathcal{D})$ Syntax $)$. Let $\mathrm{N}_{\mathrm{C}}, \mathrm{N}_{\mathrm{O}}, \mathrm{N}_{\mathrm{R}}, \mathrm{N}_{\mathrm{cF}}$ be pairwise disjoint and countably infinite sets of concept names, nominals, role names, and concrete features. Furthermore, we assume that $N_{R}$ contains a countably infinite subset $N_{a F}$ of abstract features. A 
path $u$ is a composition $f_{1} \cdots f_{n} g$ of $n$ abstract features $f_{1}, \ldots, f_{n}(n \geq 0)$ and a concrete feature $g$. Let $\mathcal{D}$ be a concrete domain. The set of $\mathcal{A L C O K}(\mathcal{D})$-concepts is the smallest set such that

- every concept name and every nominal is a concept, and

- if $C$ and $D$ are concepts, $R$ is a role name, $g$ is a concrete feature, $u_{1}, \ldots, u_{n}$ are paths, and $P \in \Phi_{\mathcal{D}}$ is a predicate of arity $n$, then the following expressions are also concepts:

$$
\neg C, C \sqcap D, C \sqcup D, \exists R . C, \forall R . C, \exists u_{1}, \ldots, u_{n} . P \text {, and } g \uparrow \text {. }
$$

A key assertion is an expression

$$
\left(u_{1}, \ldots, u_{k} \text { keyfor } C\right) \text {, }
$$

where $u_{1}, \ldots, u_{k}(k \geq 1)$ are paths and $C$ is a concept. A finite set of key assertions is called a key box.

As usual, we use $\top$ as an abbreviation for an arbitrary propositional tautology, $\perp$ as an abbreviation for $\neg \top, C \rightarrow D$ as an abbreviation for $\neg C \sqcup D$, and $C \leftrightarrow D$ as an abbreviation for $(C \rightarrow D) \sqcap(D \rightarrow C)$. Throughout this paper, we will also consider several fragments of the description logic $\mathcal{A L C O K}(\mathcal{D})$. The $\mathrm{DL} \mathcal{A L C O}(\mathcal{D})$ is obtained from $\mathcal{A} \mathcal{L C O K}(\mathcal{D})$ by admitting only empty key boxes. In particular, the set of $\mathcal{A L C O}(\mathcal{D})$-concepts is just the set of $\mathcal{A L C O K}(\mathcal{D})$-concepts. Furthermore, by disallowing the use of nominals, we obtain the fragment $\mathcal{A L C}(\mathcal{D})$ of $\mathcal{A L C O}(\mathcal{D})$ and $\mathcal{A L C K}(\mathcal{D})$ of $\mathcal{A L C O K}(\mathcal{D})$.

The description logic $\mathcal{A L C O K}(\mathcal{D})$ is equipped with a Tarski-style set-theoretic semantics. Along with the semantics, we introduce the two standard inference problems: concept satisfiability and concept subsumption.

Definition $2.3(\mathcal{A L C O K}(\mathcal{D})$ Semantics $)$. An interpretation $\mathcal{I}$ is a pair $\left(\Delta_{\mathcal{I}},{ }^{\mathcal{I}}\right)$, where $\Delta_{\mathcal{I}}$ is a non-empty set, called the domain, and ${ }^{\mathcal{I}}$ is the interpretation function. The interpretation function maps

- each concept name $C$ to a subset $C^{\mathcal{I}}$ of $\Delta_{\mathcal{I}}$,

- each nominal $N$ to a singleton subset $N^{\mathcal{I}}$ of $\Delta_{\mathcal{I}}$,

- each role name $R$ to a subset $R^{\mathcal{I}}$ of $\Delta_{\mathcal{I}} \times \Delta_{\mathcal{I}}$,

- each abstract feature $f$ to a partial function $f^{\mathcal{I}}$ from $\Delta_{\mathcal{I}}$ to $\Delta_{\mathcal{I}}$, and

- each concrete feature $g$ to a partial function $g^{\mathcal{I}}$ from $\Delta_{\mathcal{I}}$ to $\Delta_{\mathcal{D}}$. 
If $u=f_{1} \cdots f_{n} g$ is a path, then $u^{\mathcal{I}}(d)$ is defined as $g^{\mathcal{I}}\left(f_{n}^{\mathcal{I}} \cdots\left(f_{1}^{\mathcal{I}}(d)\right) \cdots\right)$. The interpretation function is extended to arbitrary concepts as follows:

$$
\begin{aligned}
(\neg C)^{\mathcal{I}} & :=\Delta_{\mathcal{I}} \backslash C^{\mathcal{I}} \\
(C \sqcap D)^{\mathcal{I}} & :=C^{\mathcal{I}} \cap D^{\mathcal{I}} \\
(C \sqcup D)^{\mathcal{I}} & :=C^{\mathcal{I}} \cup D^{\mathcal{I}} \\
(\exists R . C)^{\mathcal{I}} & :=\left\{d \in \Delta_{\mathcal{I}} \mid \text { There is } e \in \Delta_{\mathcal{I}} \text { with }(d, e) \in R^{\mathcal{I}} \text { and } e \in C^{\mathcal{I}}\right\} \\
(\forall R . C)^{\mathcal{I}} & :=\left\{d \in \Delta_{\mathcal{I}} \mid \text { For all } e \in \Delta_{\mathcal{I}}, \text { if }(d, e) \in R^{\mathcal{I}}, \text { then } e \in C^{\mathcal{I}}\right\} \\
\left(\exists u_{1}, \ldots, u_{n} . P\right)^{\mathcal{I}} & :=\left\{d \in \Delta_{\mathcal{I}} \mid \exists x_{1}, \ldots, x_{n} \in \Delta_{\mathcal{D}}: u_{i}^{\mathcal{I}}(d)=x_{i} \text { and }\left(x_{1}, \ldots, x_{n}\right) \in P^{\mathcal{D}}\right\} \\
(g \uparrow)^{\mathcal{I}} & :=\left\{d \in \Delta_{\mathcal{I}} \mid g^{\mathcal{I}}(d) \text { undefined }\right\} .
\end{aligned}
$$

Let $\mathcal{I}$ be an interpretation. Then $\mathcal{I}$ is a model of a concept $C$ iff $C^{\mathcal{I}} \neq \emptyset$. Moreover, $\mathcal{I}$ satisfies a key assertion $\left(u_{1}, \ldots, u_{n}\right.$ keyfor $\left.C\right)$ if, for any $a, b \in C^{\mathcal{I}}$,

$$
u_{1}^{\mathcal{I}}(a)=u_{1}^{\mathcal{I}}(b), \ldots, u_{n}^{\mathcal{I}}(a)=u_{n}^{\mathcal{I}}(b) \text { implies that } a=b .
$$

$\mathcal{I}$ is a model of a key box $\mathcal{K}$ iff $\mathcal{I}$ satisfies all key assertions in $\mathcal{K}$. A concept $C$ is satisfiable w.r.t. a key box $\mathcal{K}$ iff $C$ and $\mathcal{K}$ have a common model. $C$ is subsumed by a concept $D$ w.r.t. a key box $\mathcal{K}$ (written $C \sqsubseteq \mathcal{K} D$ ) iff $C^{\mathcal{I}} \subseteq D^{\mathcal{I}}$ for all models $\mathcal{I}$ of $\mathcal{K}$.

It is well-known that, in description logics providing for all Boolean operators, subsumption can be reduced to (un)satisfiability and vice versa: $C \sqsubseteq \mathcal{K} D$ iff $C \sqcap \neg D$ is unsatisfiable w.r.t. $\mathcal{K}$ and $C$ is satisfiable w.r.t. $\mathcal{K}$ iff $C \nsubseteq \mathcal{K} \perp$. This allows us to concentrate on concept satisfiability when devising complexity bounds for reasoning with description logics: lower and upper complexity bounds for concept satisfiability imply corresponding bounds for concept subsumption - only for the complementary complexity class.

If decision procedures for description logics with concrete domains are to be devised without committing to a particular concrete domain, then a well-defined interface between the decision procedure and a concrete domain reasoner is needed. Usually, this interface is based on the assumption that the concrete domain is admissible (Baader \& Hanschke, 1991a; Lutz, 2002a, 2003):

Definition 2.4 ( $\mathcal{D}$-conjunction, Admissibility). Let $\mathcal{D}$ be a concrete domain and $\mathrm{V}$ a set of variables. A $\mathcal{D}$-conjunction is a (finite) predicate conjunction of the form

$$
c=\bigwedge_{i<k}\left(x_{0}^{(i)}, \ldots, x_{n_{i}}^{(i)}\right): P_{i}
$$

where $P_{i}$ is an $n_{i}$-ary predicate for $i<k$ and the $x_{j}^{(i)}$ are variables from $\vee$. A $\mathcal{D}$-conjunction $c$ is satisfiable iff there exists a function $\delta$ mapping the variables in $c$ to elements of $\Delta_{\mathcal{D}}$ such that $\left(\delta\left(x_{0}^{(i)}\right), \ldots, \delta\left(x_{n_{i}}^{(i)}\right)\right) \in P_{i}^{\mathcal{D}}$ for each $i<k$. Such a function is called a solution for $c$. We say that the concrete domain $\mathcal{D}$ is admissible iff

1. $\Phi_{\mathcal{D}}$ contains a unary predicate $\top_{\mathcal{D}}$ such that $\top_{\mathcal{D}}^{\mathcal{D}}=\Delta_{\mathcal{D}}$

2. $\Phi_{\mathcal{D}}$ is closed under negation, i.e., for each $n$-ary predicate $P \in \Phi_{\mathcal{D}}$, there is a predicate $\bar{P} \in \Phi_{\mathcal{D}}$ of arity $n$ such that $\bar{P}^{\mathcal{D}}=\Delta_{\mathcal{D}}^{n} \backslash P^{\mathcal{D}}$ 
3. satisfiability of $\mathcal{D}$-conjunctions is decidable.

We refer to the satisfiability of $\mathcal{D}$-conjunctions as $\mathcal{D}$-satisfiability.

As we shall see, it sometimes makes a considerable difference w.r.t. complexity and decidability to restrict key boxes in various ways, for example to disallow paths of length greater than one. Therefore, we introduce some useful notions.

Definition 2.5 (Boolean, Path-free, Simple). A key box $\mathcal{K}$ is called

- Boolean if all concepts appearing in (key assertions in) $\mathcal{K}$ are Boolean combinations of concept names;

- path-free if, for all key assertions $\left(u_{1}, \ldots, u_{n}\right.$ keyfor $\left.C\right)$ in $\mathcal{K}, u_{1}, \ldots, u_{n} \in \mathrm{N}_{\mathrm{cF}}$;

- simple if it is both path-free and Boolean;

- unary if all key assertions in $\mathcal{K}$ are unary key assertions, i.e. of the form ( $u$ keyfor $C$ ).

A concept $C$ is called path-free if, in all its subconcepts of the form $\exists u_{1}, \ldots, u_{n} . P, u_{1}, \ldots, u_{n}$ are concrete features.

To emphasize that a key box might not necessarily be Boolean or path-free, we sometimes call such a key box general. Similarly, to emphasize that a key box is not necessarily a unary key box, we sometimes call such a key box composite.

\section{Lower Bounds}

In this section, we prove lower complexity bounds for description logics with concrete domains and key boxes and/or nominals. In Section 3.1, we start by showing that the satisfiability of $\mathcal{A L C K}(\mathcal{D})$-concepts w.r.t. (general) key boxes is undecidable for many interesting concrete domains. The discouraging picture painted by this result is mitigated by the fact that, in Section 4.1, we shall prove that the restriction to Boolean key boxes restores decidability. It is thus interesting to look for lower complexity bounds that apply under this restriction. In preparation for this, we introduce in Section 3.2 a NExPTIME-complete variant of the domino problem and three concrete domains that are well-suited for reductions of this problem.

In Section 3.3, we then prove that satisfiability of path-free $\mathcal{A L C K}(\mathcal{D})$-concepts w.r.t. simple key boxes is NEXPTIME-hard for a large class of concrete domains $\mathcal{D}$ and that, for many concrete domains, this holds even if we restrict key boxes to unary ones. Finally, we consider the description $\operatorname{logic} \mathcal{A L C O}(\mathcal{D})$ in Section 3.4 and identify several concrete domains such that $\mathcal{A L C O}(\mathcal{D})$-concept satisfiability (without key boxes!) is NExPTIMEhard. As we already mentioned, key boxes and nominals are closely related: key boxes can express nominals, but are in general more powerful.

\subsection{Undecidability of $\mathcal{A L C K}(\mathcal{D})$ with General Key Boxes}

We prove that satisfiability of $\mathcal{A L C K}(\mathcal{D})$-concepts w.r.t. key boxes is undecidable for a large class of concrete domains if we allow complex $\mathcal{A} \mathcal{L C K}(\mathcal{D})$-concepts to occur in key assertions. The proof is by a reduction of the well-known undecidable Post Correspondence Problem (Post, 1946; Hopcroft \& Ullman, 1979). 
Definition 3.1 (PCP). An instance $P$ of the Post Correspondence Problem (PCP) is given by a finite, non-empty list $\left(\ell_{1}, r_{1}\right), \ldots,\left(\ell_{k}, r_{k}\right)$ of pairs of words over some alphabet $\Sigma$. A sequence of integers $i_{1}, \ldots, i_{m}$, with $m \geq 1$, is called a solution for $P$ if $\ell_{i_{1}} \cdots \ell_{i_{m}}=r_{i_{1}} \cdots r_{i_{m}}$. The PCP is to decide whether a given instance $P$ has a solution.

For reducing the PCP to the satisfiability of our DLs, we need an appropriate concrete domain. It is obviously natural to use a concrete domain based on words and concatenation. We will later see that the results obtained for this concrete domain carry over to other concrete domains based on numbers and arithmetics. The following concrete domain was introduced by Lutz (2004). Its definition presupposes a fixed alphabet $\Sigma$ that is at least binary.

Definition 3.2 (Concrete domain $W$ ). The concrete domain $W$ is defined by setting $\Delta_{W}:=$ $\Sigma^{*}$ and defining $\Phi_{\mathrm{W}}$ as the smallest set containing the following predicates:

- unary predicates word and nword with word ${ }^{\mathrm{W}}=\Delta_{\mathrm{W}}$ and nword ${ }^{\mathrm{W}}=\emptyset$,

- unary predicates $={ }_{\epsilon}$ and $\neq_{\epsilon}$ with $=_{\epsilon}^{\mathrm{W}}=\{\epsilon\}$ and $\neq_{\epsilon}^{\mathrm{W}}=\Sigma^{+}$,

- a binary equality predicate $=$ and a binary inequality predicate $\neq$ with the obvious interpretation, and

- for each $w \in \Sigma^{+}$, two binary predicates conc $_{w}$ and nconc $_{w}$ with

$$
\operatorname{conc}_{w}^{\mathrm{W}}=\{(u, v) \mid v=u w\} \text { and } \operatorname{nconc}_{w}^{\mathrm{W}}=\{(u, v) \mid v \neq u w\} .
$$

It is readily checked that $W$ satisfies properties 1 and 2 of admissibility (see Definition 2.4). Moreover, $\mathbf{W}$-satisfiability is decidable:

Theorem 3.1 (Lutz, 2004). W-satisfiability is in PTime.

Thus, $\mathrm{W}$ is admissible and even of low complexity. This is important since our aim is to demonstrate that the undecidability of $\mathcal{A L C K}(\mathrm{W})$-concept satisfiability is due to the presence of keys, and not due to the high complexity of W-satisfiability.

We can now discuss the reduction of the PCP. A given instance $\left(\ell_{1}, r_{1}\right), \ldots,\left(\ell_{k}, r_{k}\right)$ is translated into an $\mathcal{A} \mathcal{L C K}(\mathcal{D})$-concept $C_{P}$ and key box $\mathcal{K}_{P}$ as defined in Figure 1 such that $P$ has a solution iff $C_{P}$ is unsatisfiable w.r.t. $\mathcal{K}_{P}$. The idea behind the reduction is that a common model of $C_{P}$ and $\mathcal{K}_{P}$ encodes all potential solutions for $P$ (i.e., sequences $i_{1}, \ldots, i_{n}$ of integers $i_{j}$ between 1 and $k$ ) and makes sure that none of them is in fact a solution. In Figure $1, f_{1}, \ldots, f_{k}$ denote abstract features while $g, \ell$, and $r$ denote concrete features. The definition of the concept Step just serves as an abbreviation and should not be confused with so-called TBoxes (see Section 4.2 for the definition of TBoxes). Models of $C_{P}$ and $\mathcal{K}_{P}$, such as the one displayed in Figure 2, have the form of an infinite $k$-ary tree whose root is connected to an "extra node" $x$ via the role $R$. Intuitively, each node of the tree represents one partial solution $i_{1}, \ldots, i_{n}$, its $\ell$-successor represents the corresponding left concatenation $\ell_{i_{1}} \cdots \ell_{i_{n}}$, and its $r$-successor the corresponding right concatenation $r_{i_{1}} \cdots r_{i_{n}}$.

To enforce the existence of the infinite tree, we employ the key box $\mathcal{K}_{P}$ : consider for example the root node's $f_{1}$-successor in Figure 2 -let us call this node $y$. Due to Line 3 


$$
\begin{aligned}
\text { Step }:= & \prod_{1 \leq i \leq k} \exists f_{i} \cdot\left(\neg A \sqcap \exists g .={ }_{\epsilon} \sqcap \exists \ell, r . \neq\right) \\
& \sqcap \prod_{1 \leq i \leq k}\left(\exists \ell, f_{i} \ell \text {. } \text { conc }_{\ell_{i}} \sqcap \exists r, f_{i} r . \text { conc }_{r_{i}}\right) \\
C_{P}:= & \exists \ell \cdot={ }_{\epsilon} \sqcap \exists r .={ }_{\epsilon} \\
& \sqcap \exists R .\left(A \sqcap \exists g .={ }_{\epsilon} \sqcap \neg \text { Step }\right) \\
& \sqcap \text { Step } \\
\mathcal{K}_{P}:= & \{g \text { keyfor } \neg \text { Step }\}
\end{aligned}
$$

Figure 1: The $\mathcal{A L C K}(\mathrm{W})$ reduction concept $C_{P}$ and key box $\mathcal{K}_{P}$.

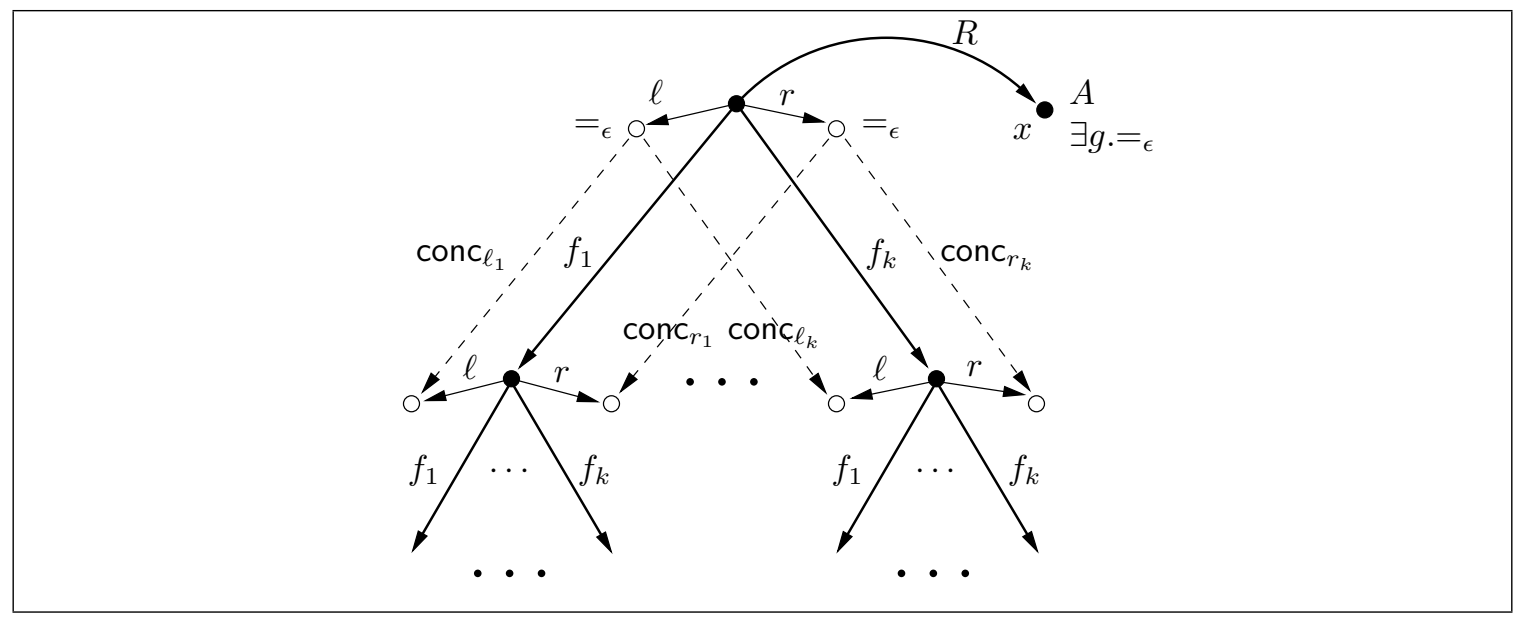

Figure 2: An example model of $C_{P}$ and $\mathcal{K}_{P}$.

of $C_{P}$ and Line 1 of Step, we have $y \in\left(\exists g .={ }_{\epsilon}\right)^{\mathcal{I}}$. Due to Line 2 of $C_{P}$, we also have $x \in\left(\exists g .{ }_{\epsilon}\right)^{\mathcal{I}}$ and $x \in(\neg \text { Step })^{\mathcal{I}}$, where $x$ is the "extra node" mentioned above. In view of the key box $\mathcal{K}_{P}$, this implies that either (i) $x=y$ or (ii) $y \in$ Step $^{\mathcal{I}}$. It is easy to see that (i) is impossible since Line 2 of $C_{P}$ and Line 1 of Step imply that $x \in A^{\mathcal{I}}$ and $y \in(\neg A)^{\mathcal{I}}$. Hence $y \in \operatorname{Step}^{\mathcal{I}}$ and, by Line 2 of Step, $y$ has the appropriate $f_{i}$-successors for $1 \leq i \leq n$. In the same way, the construction of the tree can be continued ad infinitum. The second line in the definition of Step enforces that $\ell^{\mathcal{I}}(z)=\ell_{i_{1}} \cdots \ell_{i_{n}}$ and $r^{\mathcal{I}}(z)=r_{i_{1}} \cdots r_{i_{n}}$ for $z$ an $f_{i_{1}} \cdots f_{i_{n}}$-successor of the root node. Finally, the concept $\exists \ell, r . \neq$ in Line 1 of Step implies that $\ell^{\mathcal{I}}(z) \neq r^{\mathcal{I}}(z)$ holds at all nodes $z$ of the tree (except for the root), which implies that no potential solution is a solution.

Since the size of $C_{P}$ and $\mathcal{K}_{P}$ is clearly polynomial in $k$ and the key box $\mathcal{K}_{P}$ is a unary key box, we obtain the following proposition.

Proposition 3.2. The satisfiability of $\mathcal{A L C K}(\mathrm{W})$-concepts w.r.t. (non-Boolean) path-free unary key boxes is undecidable. 
To emphasize that this undecidability result was obtained using a very simple concrete domain, let us combine Theorem 3.1 with Proposition 3.2.

Theorem 3.3. There exists a concrete domain $\mathcal{D}$ such that $\mathcal{D}$-satisfiability is in PTIME and satisfiability of $\mathcal{A L C K}(\mathcal{D})$-concepts w.r.t. (non-Boolean) path-free unary key boxes is undecidable.

At first sight, the concrete domain $\mathrm{W}$ might look artificial and one may question the relevance of lower bounds that have been obtained using W. However, it is straightforward to encode words as natural numbers and to define concatenation of words as rather simple operations on the natural numbers (Baader \& Hanschke, 1992): a word $w \neq \epsilon$ over the alphabet $\Sigma$ of cardinality $\# \Sigma$ can be interpreted as a number written at base $\# \Sigma+1$ in which the symbol that is the " 0 digit" does not occur. Hence, we can use the corresponding natural number (e.g., in base 10) to represent a word $w$, and the number 0 to represent the empty word. The concatenation of two words $v$ and $w$ can then be expressed as $v w=v \cdot(\# \Sigma+1)^{|w|}+w$, where $|w|$ denotes the length of the word $w$. Moreover, exponentiation can be expressed as multiple multiplications, multiplication as multiple additions, and addition as multiple incrementation: this is shown in Section 5.6 of (Lutz, 2004) for the case of $\mathcal{A L C}(\mathcal{D})$ extended with TBoxes (c.f. Section 4.2) and can easily be adapted to $\mathcal{A L C}(\mathcal{D})$ with non-Boolean key boxes. This observation gives rise to the following theorem:

Theorem 3.4. Let $\mathcal{D}$ be a concrete domain such that $\mathbb{N} \subseteq \Delta_{\mathcal{D}}$, $\Phi_{\mathcal{D}}$ contains a unary predicate $={ }_{0}$ with $\left(=_{0}\right)^{\mathcal{D}}=\{0\}$, binary equality and inequality predicates, and a binary predicate incr with $\operatorname{incr}^{\mathcal{D}} \cap\left\{(n, x) \mid n \in \mathbb{N}\right.$ and $\left.x \in \Delta_{\mathcal{D}}\right\}=\{(k, k+1) \mid k \in \mathbb{N}\}$. Then satisfiability of $\mathcal{A L C K}(\mathcal{D})$-concepts w.r.t. (non-Boolean) path-free unary key boxes is undecidable.

\subsection{Domino Problems and Concrete Domains}

In this section, we introduce a NEXPTIME-complete variant of the well-known, undecidable domino problem (Berger, 1966; Knuth, 1968), and then define three concrete domains $\mathrm{D}_{1}$, $\mathrm{D}_{2}$, and $\mathrm{D}_{3}$. These concrete domains will be used in Sections 3.3 and 3.4 to establish lower bounds for reasoning with $\mathcal{A L C K}(\mathcal{D})$ and Boolean key boxes, and for reasoning with $\mathcal{A L C O}(\mathcal{D})$.

In general, a domino problem is given by a finite set of tile types. Intuitively, all tile types are of the same size, each type having a square shape and colored edges. An unlimited number of tiles of each type is available. In the NExPTIME-hard variant of the domino problem that we use, the task is to tile a $2^{n+1} \times 2^{n+1}$-torus (i.e., a $2^{n+1} \times 2^{n+1}$-rectangle whose borders are "glued" together) where neighboring edges have the same color.

Definition 3.3 (Domino System). A domino system $\mathfrak{D}$ is a triple $(T, H, V)$, where $T \subsetneq \mathbb{N}$ is a finite set of tile types and $H, V \subseteq T \times T$ represent the horizontal and vertical matching conditions. Let $\mathfrak{D}$ be a domino system and $a=a_{0}, \ldots, a_{n-1}$ an initial condition, i.e. an $n$-tuple of tiles. A mapping $\tau:\left\{0, \ldots, 2^{n+1}-1\right\} \times\left\{0, \ldots, 2^{n+1}-1\right\} \rightarrow T$ is a solution for $\mathfrak{D}$ and $a$ iff, for all $x, y<2^{n+1}$, the following holds:

- if $\tau(x, y)=t$ and $\tau\left(x \oplus_{2^{n+1}} 1, y\right)=t^{\prime}$, then $\left(t, t^{\prime}\right) \in H$

- if $\tau(x, y)=t$ and $\tau\left(x, y \oplus_{2^{n+1}} 1\right)=t^{\prime}$, then $\left(t, t^{\prime}\right) \in V$ 
- $\tau(i, 0)=a_{i}$ for $i<n$.

where $\oplus_{i}$ denotes addition modulo $i$.

It follows from results in (Börger, Grädel, \& Gurevich, 1997) that the above variant of the domino problem is NEXPTIME-complete.

We define the concrete domain $D_{1}$ to be used in the reduction of the NExPTIMEcomplete domino problem to $\mathcal{A L C K}\left(\mathrm{D}_{1}\right)$-concept satisfiability w.r.t. Boolean key boxes.

Definition 3.4 (Concrete Domain $D_{1}$ ). The concrete domain $D_{1}$ is defined by setting $\Delta_{\mathrm{D}_{1}}:=\{0,1\}$ and $\Phi_{\mathrm{D}_{1}}$ to the (smallest) set containing the following predicates:

- unary predicates $T_{\mathrm{D}_{1}}$ with $\left(\top_{\mathrm{D}_{1}}\right)^{\mathrm{D}_{1}}=\Delta_{\mathrm{D}_{1}}$ and $\perp_{\mathrm{D}_{1}}$ with $\left(\perp_{\mathrm{D}_{1}}\right)^{\mathrm{D}_{1}}=\emptyset$;

- unary predicates $=_{0}$ and $={ }_{1}$ with $\left(={ }_{i}\right)^{\mathrm{D}_{1}}=\{i\}, i \in\{0,1\}$.

The second concrete domain $\mathrm{D}_{2}$ will be used for a reduction of the NExPTIME-complete domino problem to $\mathcal{A L C K}\left(\mathrm{D}_{2}\right)$-concept satisfiability w.r.t. Boolean unary key boxes. For this reduction we need to "store" vectors of bits in single concrete domain elements.

Definition 3.5 (Concrete Domain $\mathrm{D}_{2}$ ). For every $n \in \mathbb{N}$, a function $v:\{0, \ldots, n-1\} \rightarrow$ $\{0,1\}$ is called a bit vector of dimension $n$. We use $\mathrm{BV}_{n}$ to denote the set of all bit vectors of dimension $n$. The concrete domain $\mathrm{D}_{2}$ is defined by setting $\Delta_{\mathrm{D}_{2}}:=\bigcup_{i>0} \mathrm{BV}_{i}$ and $\Phi_{\mathrm{D}_{2}}$ to the (smallest) set containing the following predicates:

- unary predicates $T_{D_{2}}$ with $\left(T_{D_{2}}\right)^{D_{2}}=\Delta_{D_{2}}$ and $\perp_{D_{2}}$ with $\left(\perp_{D_{2}}\right)^{D_{2}}=\emptyset$;

- for every $k, i \in \mathbb{N}$ with $i<k$, unary predicates bit $_{k}^{i}$ and bit $1_{k}^{i}$ with

$$
\left(\operatorname{bit} n_{k}^{i}\right)^{\mathrm{D}_{2}}=\left\{v \in \Delta_{\mathrm{D}_{2}} \mid v \in \mathrm{BV}_{k} \text { and } v(i)=n\right\} \text {, }
$$

and unary predicates $\overline{\operatorname{bit} 0_{k}^{i}}$ and $\overline{\operatorname{bit} 1_{k}^{i}}$ with $\left(\overline{\operatorname{bit} n_{k}^{i}}\right)^{\mathrm{D}_{2}}=\Delta_{\mathrm{D}_{2}} \backslash\left(\operatorname{bit} n_{k}^{i}\right)^{\mathrm{D}_{2}}$.

The last concrete domain $\mathrm{D}_{3}$ is used in the reduction of the NExPTIME-complete domino problem to $\mathcal{A L C O}\left(D_{3}\right)$-concept satisfiability. In this reduction, the concrete domain $D_{3}$ contains two kinds of elements: firstly, there are elements of $\Delta_{\mathrm{D}_{3}}$ that can represent the whole $2^{n+1} \times 2^{n+1}$-torus, so-called domino arrays. Secondly, there are elements of $\Delta_{\mathrm{D}_{3}}$ that represent positions in the torus. For technical reasons to be discussed later, these elements are vectors of natural numbers rather than bit vectors, and in the following we shall just call them vectors. A domino array is then a function mapping each pair of vectors (of certain length) to a natural number which represents a tile type.

Definition 3.6 (Concrete Domain $\mathrm{D}_{3}$ ). For every $k \in \mathbb{N}$, a function $v:\{0, \ldots, k-1\} \rightarrow \mathbb{N}$ is called a vector of dimension $k$. We use $\mathrm{VE}_{k}$ to denote the set of all vectors of dimension $k$. For every $k \in \mathbb{N}$, a function $k: \mathrm{VE}_{k} \times \mathrm{VE}_{k} \rightarrow \mathbb{N}$ is called a domino array of dimension $k$. We use $\mathrm{DA}_{k}$ to denote the set of all domino arrays of dimension $k$. The concrete domain $\mathrm{D}_{3}$ is defined by setting $\Delta_{\mathrm{D}_{3}}:=\bigcup_{i>0} \mathrm{VE}_{i} \cup \bigcup_{i>0} \mathrm{DA}_{i}$ and $\Phi_{\mathrm{D}_{3}}$ to the (smallest) set containing the following predicates:

- unary predicates $T_{\mathrm{D}_{3}}$ with $\left(\top_{\mathrm{D}_{3}}\right)^{\mathrm{D}_{3}}=\Delta_{\mathrm{D}_{3}}$ and $\perp_{\mathrm{D}_{3}}$ with $\left(\perp_{\mathrm{D}_{3}}\right)^{\mathrm{D}_{3}}=\emptyset$; 
- for every $k, i \in \mathbb{N}$ with $i<k$, unary predicates $\operatorname{pos}_{k}^{i}$ and $\operatorname{pos}_{k}^{i}$ with

$$
\left(\operatorname{pos} n_{k}^{i}\right)^{\mathrm{D}_{3}}=\left\{v \in \Delta_{\mathrm{D}_{3}} \mid v \in \mathrm{VE}_{k} \text { and } v(i)=n\right\}
$$

and unary predicates $\overline{\operatorname{pos} 0_{k}^{i}}$ and $\overline{\operatorname{pos} 1_{k}^{i}}$ with $\left(\overline{\operatorname{pos} n_{k}^{i}}\right)^{\mathrm{D}_{3}}=\Delta_{\mathrm{D}_{3}} \backslash\left(\operatorname{pos} n_{k}^{i}\right)^{\mathrm{D}_{3}}$;

- for every $k, i \in \mathbb{N}$, a predicate tile ${ }_{k}^{i}$ of arity 3 with

$$
\left(\text { tile }_{k}^{i}\right)^{\mathrm{D}_{3}}=\left\{\left(v_{x}, v_{y}, d\right) \mid v_{x}, v_{y} \in \mathrm{VE}_{k}, d \in \mathrm{DA}_{k}, \text { and } d\left(v_{x}, v_{y}\right)=i\right\}
$$

and a predicate $\overline{\text { tile }_{k}^{i}}$ of arity 3 with $\left(\overline{\text { tile }_{k}^{i}}\right)^{\mathrm{D}_{3}}=\left(\Delta_{\mathrm{D}_{3}}\right)^{3} \backslash\left(\text { tile }_{k}^{i}\right)^{\mathrm{D}_{3}}$.

The reason for using vectors of natural numbers rather than bit vectors in the definition of $D_{3}$ is that we want $D_{3}$-satisfiability to be of low complexity, preferably in PTIME: consider the $\mathrm{D}_{3}$-conjunction

$$
\begin{aligned}
& \operatorname{pos}_{2}^{0}(x) \wedge \operatorname{pos}_{2}^{0}(y) \wedge \operatorname{pos}_{2}^{0}(z) \wedge \\
& \operatorname{tile}_{2}^{7}(x, v, d) \wedge \operatorname{tile}_{2}^{8}(y, v, d) \wedge \operatorname{tile}_{2}^{9}(z, v, d) .
\end{aligned}
$$

If we use bit vectors rather than vectors of natural numbers, then the upper line enforces that at least two out of the three variables $x, y$, and $z$ must take the same value. Since the value of $v$ is fixed, the lower line makes the conjunction unsatisfiable: it tries to assign the three different values $7,8,9$ to two different positions in the domino array. It seems unlikely that this kind of inconsistency can be detected in polynomial time. This problem is circumvented by using vectors of natural numbers in the definition of $D_{3}$ (but enforcing them to be bit vectors in the reduction): in this case, the above conjunction is clearly satisfiable.

Proposition 3.5. For each $i \in\{1,2,3\}$, the concrete domain $\mathrm{D}_{i}$ is admissible and satisfiability of $\mathrm{D}_{i}$-conjunctions is in PTIME.

For $\mathrm{D}_{1}$, this is trivial. For $\mathrm{D}_{2}$, a proof can be found in Appendix A. And for $\mathrm{D}_{3}$, a proof can be found in (Lutz, Areces, Horrocks, \& Sattler, 2002).

\subsection{NEXPTIME-hardness of $\mathcal{A L C K}(\mathcal{D})$ with Boolean Key Boxes}

In this section, we prove two NExPTIME-lower bounds for $\mathcal{A L C K}(\mathcal{D})$-concept satisfiability w.r.t. Boolean key boxes by reducing the NExPTIME-complete domino problem introduced in the previous section. The first reduction uses the very simple concrete domain $D_{1}$, but depends on composite key assertions. The second reduction uses the slightly more complex concrete domain $\mathrm{D}_{2}$, but only needs unary key assertions. As we will see, the two reductions yield different, incomparable results.

We first reduce the NEXPTIME-complete domino problem to $\mathcal{A L C K}\left(\mathrm{D}_{1}\right)$-concept satisfiability w.r.t. Boolean composite key boxes. Each domino system $\mathfrak{D}=(T, H, V)$ with initial condition $a=a_{0}, \ldots, a_{n-1}$ is translated into an $\mathcal{A L C K}\left(\mathrm{D}_{1}\right)$-concept $C_{\mathfrak{D}, a}$ as displayed in Figure 3. Names such as TreeX and TreeY are used as abbreviations only. We use $\forall R^{i} . C$ as an abbreviation for the $n$-fold nesting $\forall R . \cdots \forall R . C$. The names $\operatorname{xpos}_{i}$ and ypos ${ }_{i}$ used in the figure denote concrete features. In the definition of the Init concept, for each $n \in \mathbb{N}$, $\operatorname{bit}_{i}(n)$ 


$$
\begin{aligned}
& \text { TreeX : }=\exists R . X_{0} \sqcap \exists R . \neg X_{0} \sqcap \prod_{i=1 . . n} \forall R^{i} .\left(\operatorname{Dist}_{i-1} \sqcap \exists R . X_{i} \sqcap \exists R . \neg X_{i}\right) \\
& \text { TreeY }:=\operatorname{Dist}_{n} \sqcap \exists R . Y_{0} \sqcap \exists R . \neg Y_{0} \sqcap \\
& \prod_{i=1 . . n} \forall R^{i} .\left(\text { DistY }_{i-1} \sqcap \operatorname{Dist}_{n} \sqcap \exists R . Y_{i} \sqcap \exists R . \neg Y_{i}\right) \\
& \operatorname{DistX}_{k}:=\prod_{i=0 . . k}\left(\left(X_{i} \rightarrow \forall R . X_{i}\right) \sqcap\left(\neg X_{i} \rightarrow \forall R . \neg X_{i}\right)\right) \\
& \operatorname{DistY}_{k}:=\prod_{i=0 . . k}\left(\left(Y_{i} \rightarrow \forall R . Y_{i}\right) \sqcap\left(\neg Y_{i} \rightarrow \forall R . \neg Y_{i}\right)\right) \\
& \text { TransXPos }:=\prod_{i=0 . . n}\left(X_{i} \rightarrow \exists \operatorname{xpos}_{i} .={ }_{1}\right) \sqcap\left(\neg X_{i} \rightarrow \exists \operatorname{xpos}_{i} .=_{0}\right) \\
& \text { TransYPos }:=\prod_{i=0 . . n}\left(Y_{i} \rightarrow \exists \operatorname{ypos}_{i} .={ }_{1}\right) \sqcap\left(\neg Y_{i} \rightarrow \exists \operatorname{ypos}_{i} .={ }_{0}\right) \\
& \text { Succs }:=\exists R_{x} \text {.(TransXPos } \sqcap \text { TransYPos) } \sqcap \exists R_{y} \text {.(TransXPos } \sqcap \text { TransYPos) } \\
& \text { XSuccOk }:=\prod_{i=0 . . n}\left(\left(Y_{i} \rightarrow \forall R_{x} . Y_{i}\right) \sqcap\left(\neg Y_{i} \rightarrow \forall R_{x} . \neg Y_{i}\right)\right) \\
& \prod_{k=0 . . n}\left(\prod_{j=0 . . k} X_{j}\right) \rightarrow\left(\left(X_{k} \rightarrow \forall R_{x} . \neg X_{k}\right) \sqcap\left(\neg X_{k} \rightarrow \forall R_{x} . X_{k}\right)\right) \\
& \prod_{k=0 . . n}\left(\bigsqcup_{j=0 . . k} \neg X_{j}\right) \rightarrow\left(\left(X_{k} \rightarrow \forall R_{x} . X_{k}\right) \sqcap\left(\neg X_{k} \rightarrow \forall R_{x} . \neg X_{k}\right)\right) \\
& \text { YSuccOk }:=\prod_{i=0 . . n}\left(\left(X_{i} \rightarrow \forall R_{y} . X_{i}\right) \sqcap\left(\neg X_{i} \rightarrow \forall R_{y} . \neg X_{i}\right)\right) \\
& \prod_{k=0 . . n}\left(\prod_{j=0 . . k} Y_{j}\right) \rightarrow\left(\left(Y_{k} \rightarrow \forall R_{y} . \neg Y_{k}\right) \sqcap\left(\neg Y_{k} \rightarrow \forall R_{y} . Y_{k}\right)\right) \\
& \prod_{k=0 . . n}\left(\bigsqcup_{j=0 . . k} \neg Y_{j}\right) \rightarrow\left(\left(Y_{k} \rightarrow \forall R_{y} . Y_{k}\right) \sqcap\left(\neg Y_{k} \rightarrow \forall R_{y} . \neg Y_{k}\right)\right) \\
& \text { Label }:=\bigsqcup_{i \in T} D_{i} \sqcap \prod_{i, j \in T, i \neq j} \neg\left(D_{i} \sqcap D_{j}\right) \\
& \text { CheckMatch }:=\bigsqcup_{(i, j) \in H}\left(D_{i} \sqcap \forall R_{x} . D_{j}\right) \sqcap \bigsqcup_{(i, j) \in V}\left(D_{i} \sqcap \forall R_{y} \cdot D_{j}\right) \\
& \text { Init }:=\prod_{i=0 . . n-1}\left(\left(\prod_{j=0 . . n, \text { bit }_{j}(i)=0} \neg X_{j} \sqcap \prod_{j=0 . . n \text {, bit }_{j}(i)=1} X_{j} \sqcap \prod_{j=0 . . n} \neg Y_{j}\right) \rightarrow D_{a_{i}}\right) \\
& C_{\mathfrak{D}, a}:=\text { TreeX } \sqcap \forall R^{n+1} \text {. TreeY } \\
& \sqcap \forall R^{2(n+1)} \text {.(TransXPos } \sqcap \text { TransYPos } \sqcap \text { Succs } \sqcap \text { XSuccOk } \sqcap \text { YSuccOk) } \\
& \sqcap \forall R^{2(n+1)} \text {.(Label } \sqcap \text { CheckMatch } \sqcap \text { Init) }
\end{aligned}
$$

Figure 3: The $\mathcal{A L C K}\left(\mathrm{D}_{1}\right)$ reduction concept $C_{\mathfrak{D}, a}$.

is supposed to denote the $i$ 'th bit of the binary representation of $n$. We claim that $C_{\mathfrak{D}, a}$ is satisfiable w.r.t. the key box

$$
\left\{\left(\operatorname{xpos}_{0}, \ldots, \operatorname{xpos}_{n}, \operatorname{ypos}_{0}, \ldots, \operatorname{ypos}_{n} \text { keyfor } \top\right)\right\}
$$

iff there exists a solution for $\mathfrak{D}$ and $a$. To substantiate this claim, let us go through the reduction and explain the various parts of the concept $C_{\mathfrak{D}, a}$. The first step towards under- 
standing the structure of models of $C_{\mathfrak{D}, a}$ (which is the key to understanding the reduction itself) is to note that the purpose of the first line of $C_{\mathfrak{D}, a}$ is to enforce a tree structure of depth $2(n+1)$, whose leaves correspond to positions in the $2^{n+1} \times 2^{n+1}$-torus. More precisely, the TreeX concept guarantees that, in every model $\mathcal{I}$ of $C_{\mathfrak{D}, a}$, there exists a binary tree of depth $n+1$. Moreover, the DistX ${ }_{k}$ concepts (there exists one for each $k \in\{0, \ldots, n\}$ ) ensure that the leaves of this tree are binarily numbered (from 0 to $2^{n+1}-1$ ) by the concept names $X_{0}, \ldots, X_{n}$. More precisely, for a domain object $d \in \Delta_{\mathcal{I}}$, set

$$
\operatorname{xpsn}(d)=\Sigma_{i=0}^{n} \alpha_{i}(d) * 2^{i} \quad \text { where } \quad \alpha_{i}(d)= \begin{cases}1 & \text { if } d \in X_{i}^{\mathcal{I}} \\ 0 & \text { otherwise }\end{cases}
$$

The TreeX and DistX concepts ensure that there exist nodes $d_{0}, \ldots, d_{2^{n+1}-1}$ at level $n+1$ of the tree such that $\operatorname{xps}\left(d_{i}\right)=i$. Intuitively, this numbering represents the horizontal positions in the $2^{n+1} \times 2^{n+1}$-torus. The vertical positions are coded in a similar way by the $Y_{0}, \ldots, Y_{n}$ concept names. More specifically, the concepts TreeY, DistX, and DistY ensure that every $d_{i}\left(i \leq 2^{n+1}-1\right)$ is the root of another tree, in which (i) every node has the same " $X_{0}, \ldots, X_{n}$-configuration" as its root node, and (ii) the leaves are numbered binarily using the concept names $Y_{0}, \ldots, Y_{n}$ (note that the TreeY concept appears in $C_{\mathfrak{D}, a}$ inside a $\forall R^{n+1}$ value restriction). Define

$$
\operatorname{ypsn}(d)=\Sigma_{i=0}^{n} \beta_{i}(d) * 2^{i} \quad \text { where } \quad \beta_{i}(d)= \begin{cases}1 & \text { if } d \in Y_{i}^{\mathcal{I}} \\ 0 & \text { otherwise }\end{cases}
$$

In the set of leaf nodes of all the trees enforced by the TreeY concept, there exists, for each $i, j<2^{n+1}$, an object ${ }^{4} e_{i, j} \in \Delta_{\mathcal{I}} \operatorname{such}$ that $\operatorname{xpsn}\left(e_{i, j}\right)=i$ and $\operatorname{ypsn}\left(e_{i, j}\right)=j$, i.e., each $e_{i, j}$ represents the position $(i, j)$ in the $2^{n+1} \times 2^{n+1}$-torus.

The next step is to translate the individual bits of the numbering of the $e_{i, j}$-objects, which are up to now represented by concept names, into concrete domain values. This is done by the TransXPos and TransYPos concepts which ensure that, for all $\ell \leq n$, we have $\operatorname{xpos}_{\ell}^{\mathcal{I}}\left(e_{i, j}\right)=0$ if $e_{i, j} \in \neg X_{\ell}, \operatorname{xpos}_{\ell}^{\mathcal{I}}\left(e_{i, j}\right)=1$ if $e_{i, j} \in X_{\ell}$, and similarly for ypos $\operatorname{lon}_{\ell}$ and $Y_{\ell}$. Since $\mathcal{I}$ is a model for the key box

$$
\left\{\left(\operatorname{xpos}_{0}, \ldots, \operatorname{xpos}_{n}, \operatorname{ypos}_{0}, \ldots, \operatorname{ypos}_{n} \text { keyfor } \top\right)\right\}
$$

grid positions are uniquely represented by domain elements from (TransXPos $\sqcap$ TransYPos) ${ }^{\mathcal{I}}$, i.e., if $d, e \in(\text { TransXPos } \sqcap \operatorname{TransYPos})^{\mathcal{I}}$ such that $\operatorname{xpsn}(d)=\operatorname{xpsn}(e)$ and $\operatorname{ypsn}(d)=\operatorname{yxpsn}(e)$, then $d=e$. This fact is used in the concepts Succs, XSuccOk, and YSuccOk to enforce that, for the two roles $R_{x}$ and $R_{y}$ and each $i, j \leq n$, the following holds:

$$
\begin{aligned}
& R_{x}^{\mathcal{I}} \cap\left(\left\{e_{i, j}\right\} \times \Delta_{\mathcal{I}}\right)=\left\{\left(e_{i, j}, e_{\left(i \oplus_{2^{n+1}} 1\right), j}\right\}\right. \\
& R_{y}^{\mathcal{I}} \cap\left(\left\{e_{i, j}\right\} \times \Delta_{\mathcal{I}}\right)=\left\{\left(e_{i, j}, e_{i,\left(j \oplus_{2^{n+1}} 1\right)}\right\} .\right.
\end{aligned}
$$

The Succs concept ensures that, for each $e_{i, j}$, there exists an $R_{x}$-successor and an $R_{y^{-}}$ successor, and that both are in (TransXPos $\sqcap$ TransYPos) ${ }^{\mathcal{I}}$. Let $d$ be an $R_{x}$-successor of $e_{i, j}$. Then the XSuccOk concept ensures that $\operatorname{xpsn}(d)=i \oplus_{2^{n+1}} 1$ and $\operatorname{ypsn}(d)=j$. Before we

4. It does not matter if there is more than one such object. 
explain how it does this, let us note that, since all $e_{i, j}$ are in (TransXPos $\sqcap$ TransYPos) $)^{\mathcal{I}}$ and the grid positions are uniquely represented by elements of (TransXPos $\sqcap$ TransYPos) ${ }^{\mathcal{I}}$, this implies $d=e_{\left(i \oplus_{2^{n+1}} 1\right), j}$ which shows that the upper line of $(*)$ does indeed hold.

Let us now consider the XSuccOk concept in some more detail. It is essentially the DL-formulation of the well-known propositional formula

$$
\bigwedge_{k=0}^{n}\left(\bigwedge_{j=0}^{k-1} x_{j}=1\right) \rightarrow\left(x_{k}=1 \leftrightarrow x_{k}^{\prime}=0\right) \wedge \bigwedge_{k=0}^{n}\left(\bigvee_{j=0}^{k-1} x_{j}=0\right) \rightarrow\left(x_{k}=x_{k}^{\prime}\right)
$$

which encodes incrementation modulo $2^{n+1}$, i.e., if $t$ is the number (binarily) encoded by the propositional variables $x_{0}, \ldots, x_{n}$ and $t^{\prime}$ is the number encoded by the propositional variables $x_{0}^{\prime}, \ldots, x_{n}^{\prime}$, then we have $t^{\prime}=t+1$ modulo $2^{n+1}$ (see Börger et al., 1997). Taking into account the $\forall R_{x}$ quantifiers in XSuccOk, it is readily checked that this concept has just the desired effect: to ensure that, for every $R_{x}$-successor $d$ of $e_{i, j}$, we have $\operatorname{xpsn}(d)=$ $\operatorname{xpsn}\left(e_{\left(i \oplus_{2^{n+1}} 1\right), j}\right)=i \oplus_{2^{n+1}} 1$. The explanation of YSuccOk and how it enforces the lower line of $(*)$ is analogous to the $\mathrm{XSuccOk}$ case.

It remains to ensure that every grid position is labeled with precisely one tile and that the initial condition as well as the horizontal and vertical matching conditions are satisfied. The tiles are represented by concept names $D_{i}$ (where $i$ is from the set of tiles $T$ ) and the described tasks are accomplished in the standard way by the concepts Label, Init, and CheckMatch.

It is worth noting that the reduction concept is path-free and the key box is simple, i.e., path-free and Boolean. Path-freeness of concepts is often used to tame the complexity of description logics with concrete domains, although it largely sacrifices their expressive power (Lutz, 2003; Baader, Lutz, Sturm, \& Wolter, 2002b; Haarslev, Möller, \& Wessel, 2001; Horrocks \& Sattler, 2001). For example, if $\mathcal{A L C}(\mathcal{D})$ is augmented with general TBoxes, then reasoning with arbitrary concepts is undecidable while reasoning with path-free concepts is ExpTime-complete if $\mathcal{D}$ is admissible and $\mathcal{D}$-satisfiability is in ExpTime (Lutz, 2002a). This "taming approach" does not work in the presence of key boxes since, as we have just seen, satisfiability of $\mathcal{A L C}(\mathcal{D})$-concepts w.r.t. key boxes is (under some natural assumptions) NExPTImE-hard, even if both concept and key box are path-free.

Since the size of $C_{\mathfrak{D}, a}$ and of the used key box is clearly polynomial in $n$, we obtain the following proposition.

Proposition 3.6. The satisfiability of path-free $\mathcal{A L C K}\left(\mathrm{D}_{1}\right)$-concepts w.r.t. simple key boxes is NEXPTIME-hard.

It has been shown that (non path-free) $\mathcal{A L C}(\mathcal{D})$-concept satisfiability is PSPACE-complete if $\mathcal{D}$-satisfiability is in PSPACE (Lutz, 2002b). Hence, it follows from Proposition 3.5 that $\mathcal{A L C}\left(\mathrm{D}_{1}\right)$-concept satisfiability is PSPACE-complete. Thus, there is a rather dramatic increase of complexity if key boxes are added to $\mathcal{A L C}\left(\mathrm{D}_{1}\right)$. To stress that this increase is due to the key boxes themselves and not to the complexity of $D_{1}$-satisfiability, we reformulate Proposition 3.6:

Theorem 3.7. There exists a concrete domain $\mathcal{D}$ such that $\mathcal{D}$-satisfiability is in PTIME and satisfiability of path-free $\mathcal{A L C K}(\mathcal{D})$-concepts w.r.t. simple key boxes is NEXPTIME-hard. 


$$
\begin{aligned}
& \text { Succs } 2:=\exists R_{x} \text {.TransPos } \sqcap \exists R_{y} \text {.TransPos } \\
& \text { TransPos } \left.:=\prod_{i=0 . . n}\left(\left(X_{i} \rightarrow \exists \text { bv.bit } 1_{2(n+1)}^{i}\right) \sqcap \neg X_{i} \rightarrow \exists \text { bv.bit } 0_{2(n+1)}^{i}\right)\right) \sqcap \\
& \left.\prod_{i=0 . . n}\left(\left(Y_{i} \rightarrow \exists \text { bv.bit1 } 1_{2(n+1)}^{n+i+1}\right) \sqcap \neg Y_{i} \rightarrow \exists \text { bv.bit } 0_{2(n+1)}^{n+i+1}\right)\right) \\
& C_{\mathfrak{D}, a}:=\text { TreeX } \sqcap \forall R^{n+1} \text {. TreeY } \\
& \sqcap \forall R^{2(n+1)} \text {.(TransPos } \sqcap \text { Succs } 2 \sqcap \mathrm{XSuccOk} \sqcap \text { YSuccOk) } \\
& \sqcap \forall R^{2(n+1)} \text {.(Label } \sqcap \text { CheckMatch } \sqcap \text { Init) }
\end{aligned}
$$

Figure 4: The $\mathcal{A L C K}\left(\mathrm{D}_{2}\right)$ reduction concept $C_{\mathfrak{D}, a}$.

Although, due to its very low expressivity, the concrete domain $D_{1}$ itself is not very natural for knowledge representation, it is a fragment of many concrete domains that have been proposed in the literature (Baader \& Hanschke, 1992; Haarslev \& Möller, 2001; Lutz, 2003, 2002b). Indeed, the presented reduction strategy can be adapted to several "standard" concrete domains. Let us formulate a (very weak) condition that a concrete domain must satisfy in order for the presented reduction strategy to be applicable.

Theorem 3.8. Let $\mathcal{D}$ be a concrete domain. If there exist $a, b \in \Delta_{\mathcal{D}}$ with $a \neq b$ and $P_{1}, P_{2} \in$ $\Phi_{\mathcal{D}}$ such that $P_{1}^{\mathcal{D}}=\{a\}$ and $P_{2}^{\mathcal{D}}=\{b\}$, then the satisfiability of path-free $\mathcal{A} \mathcal{L C K}(\mathcal{D})$-concepts w.r.t. simple key boxes is NEXPTIME-hard.

We now present the second NExPTIME-hardness result for $\mathcal{A L C K}(\mathcal{D})$-concept satisfiability. This time, we reduce the NExPTIME-complete domino problem to the satisfiability of pathfree $\mathcal{A L C K}\left(\mathrm{D}_{2}\right)$-concepts w.r.t. simple unary key boxes. The reduction is very similar to the previous one and we only discuss the differences.

In the first reduction, we represented the individual bits of grid positions by individual concrete features $\operatorname{xpos}_{i}$ and ypos $i$ and used a composite key box to ensure that each point in the torus is represented by at most one element. In the second reduction, we use a single concrete feature bv and represent an entire position $(i, j)$ in the torus using a bit vector from the concrete domain $D_{2}$. This allows us to enforce the above mentioned uniqueness of representations using a unary key box.

The modified reduction concept $C_{\mathfrak{D}, a}$ can be found in Figure 4 , where the concepts

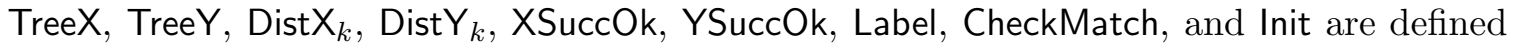
as in Figure 3. The translation of the position in the torus encoded by $X_{0}, \ldots, X_{n}, Y_{0}, \ldots, Y_{n}$ into a bit vector is done by the TransPos concept in a straightforward manner. Given what was said about the first reduction, it is not hard to see that $C_{\mathfrak{D}, a}$ is satisfiable w.r.t. the key box $\{$ (bv keyfor $T)\}$ iff there exists a solution for $\mathfrak{D}$ and $a$. We thus obtain the following proposition.

Proposition 3.9. The satisfiability of path-free $\mathcal{A L C K}\left(\mathrm{D}_{2}\right)$-concepts w.r.t. simple unary key boxes is NExPTIME-hard.

Again, we relate the NExPTime lower bound to the complexity of $D_{2}$-satisfiability, which is determined in Proposition 3.5. 
Theorem 3.10. There exists a concrete domain $\mathcal{D}$ such that $\mathcal{D}$-satisfiability is in PTIME and the satisfiability of path-free $\mathcal{A L C K}(\mathcal{D})$-concepts w.r.t. simple unary key boxes is NExPTIME-hard.

Since the elements of $\Delta_{D_{2}}$ are bit vectors, the concrete domain $D_{2}$ cannot be considered a natural choice for many application areas. But, in the reduction, $\mathrm{D}_{2}$ can be replaced by several natural concrete domains.

The central observation is that we use bit vectors only to injectively translate sequences of bits into values of the concrete domain, i.e., we translate sequences of $2(n+1)$ bits (represented by the concept names $X_{0}, \ldots, X_{n}$ and $\left.Y_{0}, \ldots, Y_{n}\right)$ into elements of $\Delta_{\mathrm{D}_{2}}$ such that, for distinct sequences, the results of the translation are also distinct. Due to this restricted use of bit vectors, there are several ways to replace them by natural numbers. For example, we can replace TransPos with the following concept TransPos' which ensures that, for each $d \in \operatorname{TransPos}^{\mathcal{I}}, s_{2 n+1}^{\mathcal{I}}(d)=\operatorname{xpsn}(d)+2^{n+1} \cdot \operatorname{ypsn}(d)$ :

$$
\begin{aligned}
& \text { TransPos }^{\prime}:= \exists \text { zero. }={ }_{0} \sqcap \prod_{i=1 \ldots 2 n+1}\left(\exists t_{i} \cdot=_{2^{i}}\right) \sqcap\left(\neg X_{0} \rightarrow \exists s_{0} \cdot=_{0}\right) \sqcap\left(X_{0} \rightarrow \exists s_{0} \cdot=_{1}\right) \sqcap \\
& \prod_{i=1 . . . n}\left(\left(\neg X_{i} \rightarrow \exists\left(s_{i-1} \text {, zero, } s_{i}\right) .+\right) \sqcap\left(X_{i} \rightarrow \exists\left(s_{i-1}, t_{i}, s_{i}\right) .+\right)\right) \sqcap \\
& \prod_{i=n+1 . .2 n+1}\left(\left(\neg Y_{i-(n+1)} \rightarrow \exists\left(s_{i-1}, \text { zero, } s_{i}\right) .+\right) \sqcap\left(Y_{i-(n+1)} \rightarrow \exists\left(s_{i-1}, t_{i}, s_{i}\right) .+\right)\right)
\end{aligned}
$$

where zero, $s_{i}$, and $t_{i}$ are concrete features, $=_{k}$ (with $k \in \mathbb{N}$ ) denotes a unary predicate with the obvious extension, and + denotes a ternary addition predicate such that, intuitively, the first two arguments are the addends and the third one is the sum.

It is easy to check that, whenever two objects $d, e \in \operatorname{TransPos}^{\mathcal{I}}$ do not agree on the interpretation of the $X_{0}, \ldots, X_{n}, Y_{0}, \ldots, Y_{n}$, then $s_{2 n+1}^{\mathcal{I}}(d) \neq s_{2 n+1}^{\mathcal{I}}(e)$, and thus the key box $\left\{\left(s_{2 n+1}\right.\right.$ keyfor $\left.\left.T\right)\right\}$ can be used for the reduction. The size of TransPos' is obviously polynomial in $n$ if the numbers $k$ appearing in $=_{k}$ predicates are coded in binary. We thus obtain the following theorem:

Theorem 3.11. Let $\mathcal{D}$ be a concrete domain such that

1. $\mathbb{N} \subseteq \Delta_{\mathcal{D}}$

2. $\Phi_{\mathcal{D}}$ contains, for each $k \in \mathbb{N}$, a predicate $={ }_{k}$ with $\left(={ }_{k}\right)^{\mathcal{D}}=\{k\}$ where the size of (the representation of $){ }_{k}$ is logarithmic in $k$, and

3. $\Phi_{\mathcal{D}}$ contains a predicate + with $(+)^{\mathcal{D}} \cap\left\{\left(k_{1}, k_{2}, x\right) \mid k_{1}, k_{2} \in \mathbb{N}\right.$ and $\left.x \in \Delta_{\mathcal{D}}\right\}=$ $\left\{\left(k_{1}, k_{2}, k_{1}+k_{2}\right) \mid k_{1}, k_{2} \in \mathbb{N}\right\}$.

Then the satisfiability of path-free $\mathcal{A} \mathcal{L C K}(\mathcal{D})$-concepts w.r.t. simple unary key boxes is NExPTIME-hard.

For example, this theorem yields NExPTIME-lower bounds for $\mathcal{A L C K}(\mathcal{D})$ instantiated with the concrete domains proposed in (Baader \& Hanschke, 1992; Haarslev \& Möller, 2001; Lutz, $2003,2002 b)$. An alternative to the addition predicate is to use multiplication to injectively 
translate sequences of bits into natural numbers. More precisely, let $p_{1}, \ldots, p_{2 n+1}$ be the first $2 n+1$ prime numbers and define another version of TransPos as follows:

$$
\begin{aligned}
\text { TransPos }^{\prime \prime}:=\exists \text { one. }={ }_{1} \sqcap \prod_{i=1 \ldots 2 n+1}\left(\exists t_{i} \cdot{ }_{p_{i}}\right) \sqcap\left(\neg X_{0} \rightarrow \exists s_{0} \cdot=_{0}\right) \sqcap\left(X_{0} \rightarrow \exists s_{0} \cdot=_{1}\right) \sqcap \\
\prod_{i=1 . . n}\left(\left(\neg X_{i} \rightarrow \exists\left(s_{i-1}, \text { one, } s_{i}\right) \cdot *\right) \sqcap\left(X_{i} \rightarrow \exists\left(s_{i-1}, t_{i}, s_{i}\right) \cdot *\right)\right) \sqcap \\
\prod_{i=n+1 . .2 n+1}\left(\left(\neg Y_{i-(n+1)} \rightarrow \exists\left(s_{i-1}, \text { one }, s_{i}\right) \cdot *\right) \sqcap\left(Y_{i-(n+1)} \rightarrow \exists\left(s_{i-1}, t_{i}, s_{i}\right) \cdot *\right)\right)
\end{aligned}
$$

where $*$ is a ternary multiplication predicate.

Since the factorization of natural numbers into prime numbers is unique, we can again use the key box $\left\{\left(s_{2 n+1}\right.\right.$ keyfor $\left.\left.T\right)\right\}$ for the reduction. Moreover, it is well-known that the $k$ 'th prime is polynomial in $k$ (Graham, Knuth, \& Patashnik, 1990), and thus the size of the concept TransPos" is polynomial in $n$ even if the numbers $k$ in $={ }_{k}$ predicates are coded unarily. We thus obtain another theorem concerning quite natural concrete domains:

Theorem 3.12. Let $\mathcal{D}$ be a concrete domain such that

1. $\mathbb{N} \subseteq \Delta_{\mathcal{D}}$

2. $\Phi_{\mathcal{D}}$ contains, for each $k \in \mathbb{N}$, a predicate $=_{k}$ with $\left(={ }_{k}\right)^{\mathcal{D}}=\{k\}$, and

3. $\Phi_{\mathcal{D}}$ contains a predicate $*$ with $(*)^{\mathcal{D}} \cap\left\{\left(k_{1}, k_{2}, x\right) \mid k_{1}, k_{2} \in \mathbb{N}\right.$ and $\left.x \in \Delta_{\mathcal{D}}\right\}=$ $\left\{\left(k_{1}, k_{2}, k_{1} \cdot k_{2}\right) \mid k_{1}, k_{2} \in \mathbb{N}\right\}$.

Then the satisfiability of path-free $\mathcal{A} \mathcal{L C K}(\mathcal{D})$-concepts w.r.t. simple unary key boxes is NEXPTIME-hard.

\subsection{NEXPTime-hardness of $\mathcal{A L C O}(\mathcal{D})$}

As we already pointed out in Section 1, the relationship between key boxes and nominals is rather close: the latter can be "simulated" by the former if the concrete domain provides predicates that can be used to uniquely describe elements of $\Delta_{\mathcal{D}}$. For example, in $\mathcal{A} \mathcal{L C K}\left(\mathrm{D}_{1}\right)$ the concept $\exists g .=_{0}$ behaves as a nominal if we use the key assertion ( $g$ keyfor $\top$ ). We can even define $n$ nominals using $n$ single concrete features in unary-key assertions. In the logics $\mathcal{A L C K}\left(\mathrm{D}_{2}\right)$ and $\mathcal{A L C K}\left(\mathrm{D}_{3}\right)$, a single concrete feature and unary key assertions are sufficient to simulate an arbitrary number of nominals: for example, in $\mathcal{A L C K}\left(\mathrm{D}_{2}\right)$ the concept $C=\exists g$.bit $0_{2}^{0} \sqcap \exists g$.bit $1_{2}^{1}$ uniquely describes the bit vector $(0,1) \in \mathrm{BV}_{2} \subseteq \Delta_{\mathrm{D}_{2}}$, i.e., $a \in C^{\mathcal{I}}$ implies $g^{\mathcal{I}}(a)=(0,1)$. Obviously, any other bit vector (of any length!) can be described in a similar way.

This illustrates that, for most non-trivial concrete domains $\mathcal{D}$, the logic $\mathcal{A L C K}(\mathcal{D})$ is (at least) as expressive as $\mathcal{A L C O}(\mathcal{D})$. Although the converse does not hold, the expressive power of $\mathcal{A L C O}(\mathcal{D})$ is still sufficient to prove NExPTImE-hardness of concept satisfiability, provided that a suitable concrete domain $\mathcal{D}$ is used. Since $\mathcal{A L C O}$ concept satisfiability is PSPACE-complete (Areces, Blackburn, \& Marx, 1999), this is yet another example of a DL where an even seemingly harmless extension with concrete domains has a dramatic effect on the computational complexity (Lutz, 2003). 


$$
\begin{aligned}
& \text { Nominal }:=\exists f \cdot N \\
& \text { XSucc }:=\prod_{k=0 . . n}\left(\prod_{j=0 . . k} X_{j}\right) \rightarrow\left(X_{k} \leftrightarrow \neg X_{k}^{\prime}\right) \sqcap \prod_{k=0 . . n}\left(\bigsqcup_{j=0 . . k}^{\bigsqcup_{j}} \neg X_{j}\right) \rightarrow\left(X_{k} \leftrightarrow X_{k}^{\prime}\right) \\
& \text { YSucc }:=\prod_{k=0 . . n}\left(\prod_{j=0 . . k} Y_{j}\right) \rightarrow\left(Y_{k} \leftrightarrow \neg Y_{k}^{\prime}\right) \sqcap \prod_{k=0 . . n}\left(\bigsqcup_{j=0 . . k} \neg Y_{j}\right) \rightarrow\left(Y_{k} \leftrightarrow Y_{k}^{\prime}\right) \\
& \text { TransXPos }:=\prod_{i=0 . . n}\left(\left(X_{i} \rightarrow \exists \mathrm{bvx} \cdot \operatorname{pos} 1_{n+1}^{i}\right) \sqcap\left(\neg X_{i} \rightarrow \exists \mathrm{bvx} \cdot \operatorname{pos} 0_{n+1}^{i}\right)\right) \\
& \text { TransYPos }:=\prod_{i=0 . . n}\left(\left(Y_{i} \rightarrow \exists \text { bvy.pos } 1_{n+1}^{i}\right) \sqcap\left(\neg Y_{i} \rightarrow \exists \text { bvy.pos } 0_{n+1}^{i}\right)\right) \\
& \text { TransXSucc }:=\prod_{i=0 . . n}\left(\left(X_{i}^{\prime} \rightarrow \exists \text { bvxs.pos } 1_{n+1}^{i}\right) \sqcap\left(\neg X_{i}^{\prime} \rightarrow \exists \text { bvxs.pos} 0_{n+1}^{i}\right)\right) \\
& \text { TransYSucc }:=\prod_{i=0 . . n}\left(\left(Y_{i}^{\prime} \rightarrow \exists \text { bvys.pos } 1_{n+1}^{i}\right) \sqcap\left(\neg Y_{i}^{\prime} \rightarrow \exists \text { bvys.pos } 0_{n+1}^{i}\right)\right) \\
& \text { CheckHMatch }:=\bigsqcup_{i, j \in H}\left(\exists(\text { bvx, bvy, } f \circ \text { darr }) \cdot \text { tile }_{n+1}^{i} \sqcap \exists(\text { bvxs, bvy, } f \circ \text { darr }) \cdot \text { tile }_{n+1}^{j}\right) \\
& \text { CheckVMatch }:=\bigsqcup_{i, j \in V}\left(\exists(\text { bvx, bvy }, f \circ \text { darr }) . \text { tile }{ }_{n+1}^{i} \sqcap \exists(\text { bvx, bvys, } f \circ \text { darr }) . \text { tile }_{n+1}^{j}\right) \\
& \text { Init2 }:=\prod_{i=0 . . n-1}\left(\left(\prod_{j=0 . . n, \text { bit }_{j}(i)=0} \neg X_{j} \sqcap \prod_{j=0 . . n, \text { bit }_{j}(i)=1} X_{j} \sqcap \prod_{j=0 . . n} \neg Y_{j}\right)\right. \\
& \left.\rightarrow \exists(\text { bvx, bvy, } f \circ \text { darr }) . \text { tile } a_{n+1}^{a_{i}}\right) \\
& C_{\mathfrak{D}, a}:=\text { TreeX } \sqcap \forall R^{n+1} \text {. TreeY } \sqcap \forall R^{2(n+1)} \text {. Nominal } \sqcap \\
& \forall R^{2(n+1)} \text {. (TransXPos } \sqcap \text { TransYPos } \sqcap \\
& \text { XSucc } \sqcap \text { YSucc } \sqcap \text { TransXSucc } \sqcap \text { TransYSucc } \sqcap \\
& \text { Init2 } \sqcap \text { CheckHMatch } \sqcap \text { CheckVMatch) }
\end{aligned}
$$

Figure 5: The $\mathcal{A L C O}\left(\mathrm{D}_{3}\right)$ reduction concept $C_{\mathfrak{D}, a}$.

In this section, we reduce the NExpTime-complete domino-problem to $\mathcal{A L C O}\left(\mathrm{D}_{3}\right)$ concept satisfiability. Again, let $\mathfrak{D}=(T, H, V)$ be a domino system and $a=a_{0}, \ldots, a_{n-1}$ an initial condition. The modified reduction concept $C_{\mathfrak{D}, a}$ is defined in Figure 5 , where bvx, bvy, bvxs, bvys, and darr denote concrete features, $N$ denotes a nominal, and the concepts TreeX, TreeY, DistX ${ }_{k}$, and Dist $Y_{k}$ are defined as in Figure 3. As in the previous reductions, we now give a detailed explanation of the reduction strategy to show that $C_{\mathfrak{D}, a}$ is satisfiable iff there exists a solution for $\mathfrak{D}$ and $a$. Formal details can then easily be worked out by the interested reader.

Let $\mathcal{I}$ be a model for $C_{\mathfrak{D}, a}$. To explain the structure of $\mathcal{I}$, it is convenient to start with the first line of $C_{\mathfrak{D}, a}$. As in the previous reductions, the TreeX and TreeY concepts are used to ensure that $\mathcal{I}$ contains a tree-shaped substructure of depth $n+1$ whose leaf nodes are the roots of additional trees of depth $n+1$ such that the set of the leafs of the 


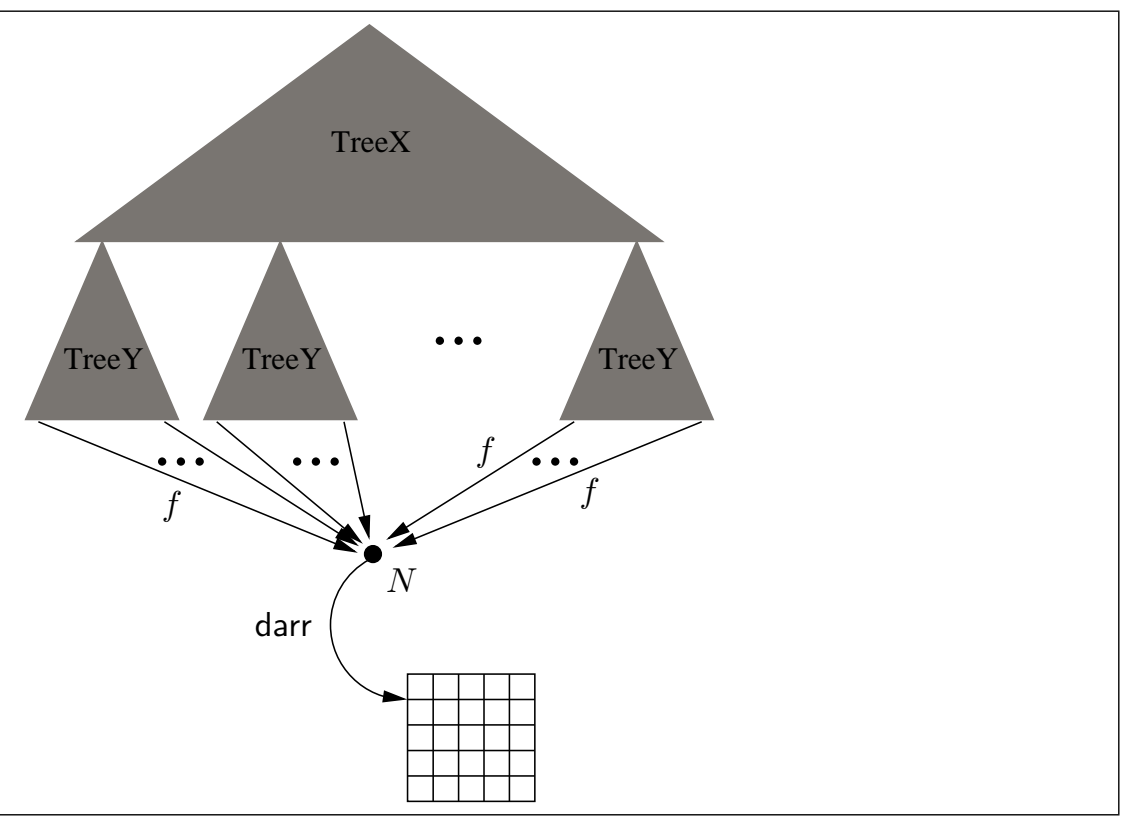

Figure 6: The structure of models of $C_{\mathfrak{D}, a}$.

latter trees correspond to the positions in the $2^{n+1} \times 2^{n+1}$-torus, i.e., for each position, there is a leaf node representing it. The torus positions are binarily encoded by the concept names $X_{0}, \ldots, X_{n}$ and $Y_{0}, \ldots, Y_{n}$ and we use $e_{i, j}$ to refer to the leaf with $\operatorname{xpsn}\left(e_{i, j}\right)=i$ and $\operatorname{ypsn}\left(e_{i, j}\right)=j($ see Section 3.3).

As in the previous reductions, the numbers coded by $X_{0}, \ldots, X_{n}$ and $Y_{0}, \ldots, Y_{n}$ are translated into concrete domain values, which is done by the TransXPos and TransYPos concepts. Note that, in contrast to the $\mathcal{A L C K}\left(\mathrm{D}_{2}\right)$-reduction, the x-position and the $\mathrm{y}$ position are not stored in the same bit vector, but rather in the two distinct ones bvx and bvy. Also in contrast to the previous reduction, the actual tiling of the torus is not represented by the leaf nodes $e_{i, j}$, but rather by a domino array: the last conjunct in the first line of $C_{\mathfrak{D}, a}$ ensures that every leaf $e_{i, j}$ is connected via the abstract feature $f$ to the (unique) element $w \in N^{\mathcal{I}}$.

The domain element $w$ is associated with a domino array via the concrete feature darr (as we shall see later, this is guaranteed by the CheckHMatch and CheckVMatch concepts). This domino array represents the tiling of the $2^{n+1} \times 2^{n+1}$-torus. Summing up, the structure of $\mathcal{I}$ is roughly as shown in Figure 6.

Since the tiling is stored in a domino array, we need to explain the purpose of the leaf nodes $e_{i, j}$ : these nodes are used to enforce the initial condition and the horizontal and vertical matching condition. Let us discuss the horizontal matching condition (the vertical matching condition is enforced analogously): the XSucc concept is the DL reformulation of the propositional logic formula for incrementation modulo $2^{n+1}$ and ensures that, for each $e_{i, j}$, the concept names $X_{0}^{\prime}, \ldots, X_{n}^{\prime}$ encode the number $i \oplus_{2^{n+1}} 1$, i.e., the horizontal position of $e_{i, j}$ 's horizontal neighbor. In addition to the storage of the horizontal and vertical position of $e_{i, j}$ in $\operatorname{bvx}\left(e_{i, j}\right)$ and $\operatorname{bvy}\left(e_{i, j}\right)$, we also store the horizontal position $i \oplus_{2^{n+1}} 1$ of $e_{i, j}$ 's horizontal successor in $\operatorname{bvxs}\left(e_{i, j}\right)$. Finally, CheckHMatch verifies that the tiles at positions 
$(i, j)$ and $\left(i \oplus_{2^{n+1}} 1, j\right)$, which are both stored in the domino array, are compatible with the horizontal matching condition.

Note that CheckHMatch also ensures that the domain element $w$ (with $\{w\}=N^{\mathcal{I}}$ ) has a domino array attached via the concrete feature darr and that, for each position $(i, j)$, the (unique!) tile stored in the domino array is from the set $T$. The initial condition is ensured via the Init2 concept in a similar way. We (again) use bit $_{j}(i)$ to denote the $j$ 'th bit of the binary encoding of the natural number $i$.

Using the above considerations, the correctness of the reduction is readily checked. Moreover, the size of $C_{\mathfrak{D}, a}$ is at most polynomial in $n$. Note that $C_{\mathfrak{D}, a}$ is not path-free: paths of length two appear in the concepts CheckHMatch, CheckVMatch, and Init2. Summing up, the reduction described yields the following result:

Proposition 3.13. The satisfiability of $\mathcal{A L C O}\left(\mathrm{D}_{3}\right)$-concepts is NExPTIME-hard.

Again, we relate the NExPTime lower bound to the complexity of $D_{3}$-satisfiability, which is determined in Proposition 3.5.

Theorem 3.14. There exists a concrete domain $\mathcal{D}$ such that $\mathcal{D}$-satisfiability is in PTIME and the satisfiability of $\mathcal{A L C O}(\mathcal{D})$-concepts is NExPTIME-hard.

Note that the reduction uses only a single nominal $N$. This is a dramatic increase of complexity since it has been shown that satisfiability of $\mathcal{A L C}(\mathcal{D})$-concepts (i.e., without nominals and key boxes) is PSPACE-complete provided that $\mathcal{D}$ is admissible and $\mathcal{D}$-satisfiability is in PSPACE (Lutz, 2002b).

As in previous sections, we note that $D_{3}$ can be replaced by more natural concrete domains in the NExPTIme-hardness proof presented. The idea is to represent the whole domino array by a single natural number and then to use arithmetic operations to access the individual positions: a natural number $k$ can be viewed as a domino array by partitioning its binary representation into $2^{n+1} \cdot 2^{n+1}=2^{2(n+1)}$ "sections" of length $\lceil\log (\# T)\rceil$, where $\# T$ denotes the cardinality of the set of tile types $T$. Each such section describes the tile of a single position in the torus. The sections can be accessed by using integer division and reminder operations: if $k$ is the natural number representing the torus, then the tile of posisition $i$ is computed by

$$
\left(k \operatorname{div} 2^{i \cdot\lceil\log (\# T)\rceil}\right) \bmod 2^{\lceil\log (\# T)\rceil}+1 .
$$

Thus, we introduce ternary predicates div for integer division and mod for computing the remainder of a division, and a binary predicate $2^{\mathrm{x}}$ expressing exponentiation with basis 2 . Then we modify the reduction as follows: we replace TransXPos and TransYPos by the TransPos' concept from Section 3.3 to translate the two numbers encoded by $X_{1}, \ldots, X_{n}$ and $Y_{1}, \ldots, Y_{n}$ into a single natural number that is stored in the concrete feature $s_{2 n+1}$. We then devise a new concept Tile $[i]$ (for each $i \in T$ ) enforcing that the position identified by the feature $s_{2 n+1}$ is labeled with tile $i$ :

$$
\begin{aligned}
\text { Tile }[i]:= & \exists r .=\lceil\log (\# T)\rceil \sqcap \exists s_{2 n+1}, r, r^{\prime} \cdot * \sqcap \exists r^{\prime}, r^{\prime \prime} .2^{\times} \sqcap \exists \text { one. }={ }_{1} \sqcap \exists r, \text { one }, t .+\sqcap \exists t, t^{\prime} .2^{\times} \\
& \sqcap \exists f \text { torus, } r^{\prime \prime}, u \cdot \operatorname{div} \sqcap \exists u, t^{\prime \prime}, \text { tile. } \bmod \sqcap \exists \text { tile. }={ }_{i} .
\end{aligned}
$$


Here, $r, r^{\prime}, r^{\prime \prime}, t, t^{\prime}, u$, one, torus, and tile are concrete features. The torus feature is the counterpart of the darr feature in the original reduction, i.e., it stores the natural number that represents the tiling array. We can use the Tile $[i]$ concept in the obvious way inside the CheckHMatch, CheckVMatch, and Init2 concepts. The size of the resulting reduction concept is polynomial in $n$ if the numbers $k$ appearing in $=_{k}$ predicates are coded in binary. We thus obtain the following theorem:

Theorem 3.15. Let $\mathcal{D}$ be a concrete domain such that

1. $\mathbb{N} \subseteq \Delta_{\mathcal{D}}$,

2. $\Phi_{\mathcal{D}}$ contains the predicates a predicate $=_{k}($ for each $k \in \mathbb{N}), 2^{\mathrm{x}},+, *$, div, mod with the following extensions

$$
\begin{aligned}
\left(=_{k}\right)^{\mathcal{D}} & =\{k\} \\
\left(2^{\times}\right)^{\mathcal{D}} \cap\left\{(k, x) \mid k \in \mathbb{N} \text { and } x \in \Delta_{\mathcal{D}}\right\} & =\left\{\left(k, 2^{k}\right) \mid k \in \mathbb{N}\right\} \\
(+)^{\mathcal{D}} \cap\left\{\left(k_{1}, k_{2}, x\right) \mid k_{1}, k_{2} \in \mathbb{N} \text { and } x \in \Delta_{\mathcal{D}}\right\} & =\left\{\left(k_{1}, k_{2}, k_{1}+k_{2}\right) \mid k_{1}, k_{2} \in \mathbb{N}\right\} \\
(*)^{\mathcal{D}} \cap\left\{\left(k_{1}, k_{2}, x\right) \mid k_{1}, k_{2} \in \mathbb{N} \text { and } x \in \Delta_{\mathcal{D}}\right\} & =\left\{\left(k_{1}, k_{2}, k_{1} \cdot k_{2}\right) \mid k_{1}, k_{2} \in \mathbb{N}\right\} \\
(\text { div })^{\mathcal{D}} \cap\left\{\left(k_{1}, k_{2}, x\right) \mid k_{1}, k_{2} \in \mathbb{N} \text { and } x \in \Delta_{\mathcal{D}}\right\} & =\left\{\left(k_{1}, k_{2}, k_{1} \operatorname{div} k_{2}\right) \mid k_{1}, k_{2} \in \mathbb{N}\right\} \\
(\bmod )^{\mathcal{D}} \cap\left\{\left(k_{1}, k_{2}, x\right) \mid k_{1}, k_{2} \in \mathbb{N} \text { and } x \in \Delta_{\mathcal{D}}\right\} & =\left\{\left(k_{1}, k_{2}, k_{1} \bmod k_{2}\right) \mid k_{1}, k_{2} \in \mathbb{N}\right\}
\end{aligned}
$$

Then the satisfiability of $\mathcal{A L C O}(\mathcal{D})$-concepts is NExPTIME-hard.

\section{Reasoning Procedures}

This section is devoted to developing reasoning procedures for DLs with concrete domains, nominals, and keys. We start with devising a tableau algorithm that decides the satisfiability of $\mathcal{A L C O K}(\mathcal{D})$-concepts w.r.t. Boolean key boxes. This algorithm yields a NExPTIme upper complexity bound matching the lower bounds established in Section 3.3.

Then we consider the rather powerful description logic $\mathcal{S H O} \mathcal{Q K}(\mathcal{D})$. This DL, which is an extension of $\mathcal{S H O Q}(\mathcal{D})$ (Horrocks \& Sattler, 2001; Pan \& Horrocks, 2002), provides a wealth of expressive means such as transitive roles, role hierarchies, nominals, and qualifying number restrictions. Moreover, $\mathcal{S H O} \mathcal{Q K}(\mathcal{D})$ is equipped with a restricted variant of the concrete domain constructor and with key boxes. We develop a tableau algorithm for deciding the satisfiability of $\mathcal{S H O} \mathcal{Q K}(\mathcal{D})$-concepts w.r.t. path-free key boxes. Due to the restrictedness of $\mathcal{S H O} \mathcal{Q K}(\mathcal{D})$ 's concrete domain constructor, we can even admit general rather than only Boolean key boxes. Again, the algorithm yields a tight NExPTIME upper complexity bound.

\subsection{A Tableau Algorithm for $\mathcal{A L C O K}(\mathcal{D})$ with Boolean Key Boxes}

Tableau algorithms decide the satisfiability of the input concept (in our case w.r.t. the input key box) by attempting to construct a model for it. More precisely, a tableau algorithm starts with an initial data structure induced by the input concept and then repeatedly applies so-called completion rules to it. This rule application can be thought of as attempting to construct a model for the input concept. Finally, either the algorithm will find an obvious contradiction or it will encounter a situation that is contradiction-free and in which no 
more completion rules are applicable. In the former case, the input concept is unsatisfiable, while it is satisfiable in the latter.

When devising a tableau algorithm for a description logic with concrete domains but without committing to a particular concrete domain, it is commonly assumed that the concrete domain is admissible, which implies decidability of the satisfiability of $\mathcal{D}$-conjunctions. In the presence of keys, however, this is not enough: if a $\mathcal{D}$-conjunction is satisfiable, we also want to know which of its variables take the same values in an arbitrary but fixed solution. As an example, consider the concrete domain $\mathbb{N}=\left(\mathbb{N},\left\{<_{n} \mid n \in \mathbb{N}\right\}\right)$ and the $\mathrm{N}$-conjunction

$$
c=<_{2}\left(v_{1}\right) \wedge<_{2}\left(v_{2}\right) \wedge<_{2}\left(v_{3}\right) .
$$

Obviously, one solution $\delta$ for $c$ satisfies $\delta\left(v_{1}\right)=\delta\left(v_{2}\right)$, another satisfies $\delta\left(v_{1}\right)=\delta\left(v_{3}\right)$, and so on. Our tableau algorithm uses such identity information passed from the concrete domain reasoner since, in the presence of key boxes, it can have an impact on the structure of the constructed model. For example, this information reveals the unsatisfiability of

$$
\left.\exists R . A \sqcap \exists R .(\neg A \sqcap B) \sqcap \exists R .(\neg A \sqcap \neg B) \sqcap \forall R . \exists g .<_{2} \text { w.r.t. ( } g \text { keyfor } \top\right) \text {. }
$$

To formalize this requirement, we strengthen the notion of admissibility into key-admissibility. Since the tableau algorithm developed in this section is non-deterministic, we formulate keyadmissibility in a non-deterministic way.

Definition 4.1 (Key-admissible). A concrete domain $\mathcal{D}$ is key-admissible iff it satisfies the following properties:

1. $\Phi_{\mathcal{D}}$ contains a name $\top_{\mathcal{D}}$ for $\Delta_{\mathcal{D}}$;

2. $\Phi_{\mathcal{D}}$ is closed under negation;

3. there exists an algorithm that takes as input a $\mathcal{D}$-conjunction $c$, returns clash if $c$ is unsatisfiable, and otherwise non-deterministically outputs an equivalence relation $\sim$ on the set of variables $V$ used in $c$ such that there exists a solution $\delta$ for $c$ with the following property: for all $v, v^{\prime} \in V$

$$
\delta(v)=\delta\left(v^{\prime}\right) \text { iff } v \sim v^{\prime}
$$

An algorithm showing the behaviour described in item 3 above is called a $\mathcal{D}$-tester, and the equivalence relations $\sim$ are called concrete equivalences. We say that extended $\mathcal{D}$ satisfiability is in $N P$ if there exists a $\mathcal{D}$-tester running in polynomial time.

Please note that key-admissibility is less esoteric than it might seem: any concrete domain that is admissible and provides for an equality predicate is also key-admissible. Due to admissibility, the presence of an equality predicate implies that an inequality predicate is also available. We can thus construct a $\mathcal{D}$-tester from an algorithm for $\mathcal{D}$-satisfiability: when presented with a predicate conjunction $c$, we simply "guess" an equivalence relation $\sim$ on the set of variables used in $c$. Then we decide the (non-extended) satisfiability of the conjunction $c \wedge \bigwedge_{v \sim v^{\prime}}=\left(v, v^{\prime}\right) \wedge \bigwedge_{v \psi_{v^{\prime}}} \neq\left(v, v^{\prime}\right)$, return clash if it is unsatisfiable and $\sim$ otherwise. The rather weak condition that an equality predicate should be present is 


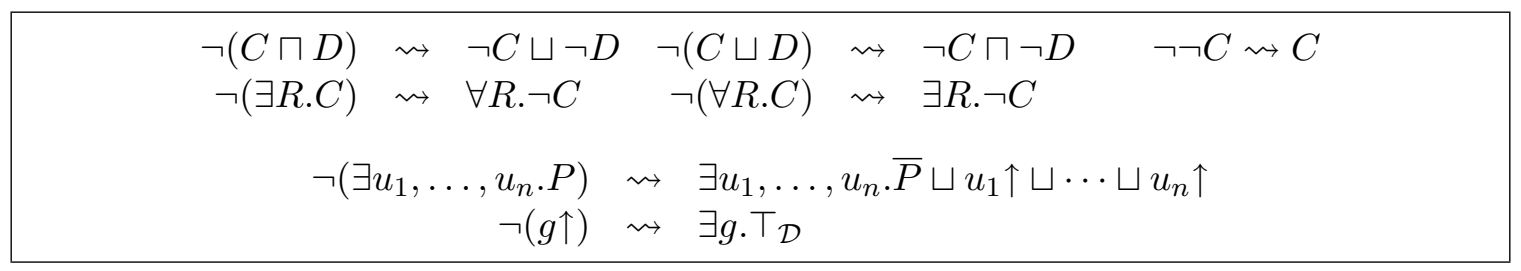

Figure 7: The NNF rewrite rules.

satisfied by almost all concrete domains proposed in the literature (see, e.g. (Lutz, 2003; Baader \& Hanschke, 1991b; Kamp \& Wache, 1996; Haarslev, Lutz, \& Möller, 1998; Baader \& Sattler, 1998)).

Throughout this chapter, we assume that any concrete domain is equipped with an equality predicate. This assumption is w.l.o.g. since any $\mathcal{D}$-conjunction using equality can be translated into an equivalent one without equality by identifying variables according to the stated equalities. This assumption must not be confused with what was discussed in the previous paragraph: even if the concrete domain $\mathcal{D}$ is admissible and its set of predicates is thus closed under negation, this assumption does not imply the presence of an inequality predicate.

We need some more prerequisites before we can start the presentation of the tableau algorithm: a concept is in negation normal form (NNF) if negation occurs only in front of concept names and nominals. It is easily seen that, if the concrete domain $\mathcal{D}$ is admissible, then every $\mathcal{A L C O K}(\mathcal{D})$-concept can be converted into an equivalent one in NNF by exhaustively applying the rewrite rules displayed in Figure 7 . We use $\dot{\neg} C$ to denote the result of converting $\neg C$ to NNF. A key box is in NNF if all concepts occurring in key assertions are in NNF. In what follows, we generally assume input concepts and key boxes to be in NNF. Let $C$ be an $\mathcal{A L C O K}(\mathcal{D})$-concept and $\mathcal{K}$ a key box. We use $\operatorname{sub}(C)$ to denote the set of subconcepts of $C$ (including $C$ itself) and $\operatorname{con}(\mathcal{K})$ to denote the set of concepts appearing on the right-hand side of key assertions in $\mathcal{K}$. For a set of concepts $\Gamma, \operatorname{sub}(\Gamma)$ denotes the set $\bigcup_{C \in \Gamma} \operatorname{sub}(C)$. Moreover, we write $\mathrm{cl}(C, \mathcal{K})$ as abbreviation for the set

$$
\operatorname{sub}(C) \cup \operatorname{sub}(\operatorname{con}(\mathcal{K})) \cup\{\dot{\neg} D \mid D \in \operatorname{sub}(\operatorname{con}(\mathcal{K}))\}
$$

We now start the presentation of the tableau algorithm by introducing the underlying data structure.

Definition 4.2 (Completion System). Let $\mathrm{O}_{a}$ and $\mathrm{O}_{c}$ be disjoint and countably infinite sets of abstract and concrete nodes. A completion tree for an $\mathcal{A L C O K}(\mathcal{D})$-concept $C$ and a key box $\mathcal{K}$ is a finite, labeled tree $\mathbf{T}=\left(V_{a}, V_{c}, E, \mathcal{L}\right)$ with nodes $V_{a} \cup V_{c}$ such that $V_{a} \subseteq \mathrm{O}_{\mathrm{a}}$, $V_{c} \subseteq \mathrm{O}_{\mathrm{c}}$, and all nodes from $V_{c}$ are leaves. The tree is labeled as follows:

- each node $a \in V_{a}$ is labeled with a subset $\mathcal{L}(a)$ of $\operatorname{cl}(C, \mathcal{K})$;

- each edge $(a, b) \in E$ with $a, b \in V_{a}$ is labeled with a role name $\mathcal{L}(a, b)$ occurring in $C$ or $\mathcal{K}$;

- each edge $(a, x) \in E$ with $a \in V_{a}$ and $x \in V_{c}$ is labeled with a concrete feature $\mathcal{L}(a, x)$ occurring in $C$ or $\mathcal{K}$. 
For $a \in V_{a}$, we use $\operatorname{lev}_{\mathbf{T}}(a)$ to denote the depth at which $a$ occurs in $\mathbf{T}$ (starting with the root node on depth 0). A completion system for an $\mathcal{A L C O K}(\mathcal{D})$-concept $C$ and a key box $\mathcal{K}$ is a tuple $(\mathbf{T}, \mathcal{P}, \prec, \sim)$, where

- $\mathbf{T}=\left(V_{a}, V_{c}, E, \mathcal{L}\right)$ is a completion tree for $C$ and $\mathcal{K}$,

- $\mathcal{P}$ is a function mapping each $P \in \Phi_{\mathcal{D}}$ of arity $n$ in $C$ to a subset of $V_{c}^{n}$,

- $\prec$ is a linear ordering of $V_{a}$ such that $\operatorname{lev}_{\mathbf{T}}(a) \leq \operatorname{lev}_{\mathbf{T}}(b)$ implies $a \prec b$, and

- $\sim$ is an equivalence relation on $V_{c}$.

Let $\left(V_{a}, V_{c}, E, \mathcal{L}\right)$ be a completion tree. A node $b \in V_{a}$ is an $R$-successor of a node $a \in V_{a}$ if $(a, b) \in E$ and $\mathcal{L}(a, b)=R$, while a node $x \in V_{c}$ is a $g$-successor of $a$ if $(a, x) \in E$ and $\mathcal{L}(a, x)=g$. For a path $u$, the notion of $u$-successor is defined in the obvious way.

Intuitively, the relation $\sim$ records equalities between concrete nodes found during the (non-deterministic) model construction process. The recording is necessary since equalities between concrete nodes can induce equalities between abstract nodes which, in turn, can imply more equalities between concrete nodes. This can be seen in the following example: assume the completion tree contains, for $i \in\{1,2\}$, an abstract node $a_{i}$ with a concrete $g$-successor $x_{i}$ and a concrete $g^{\prime}$-successor $y_{i}$. Now assume that the key box contains ( $g$ keyfor $\top$ ), and that the $\mathcal{D}$-tester returns $x_{1} \sim x_{2}$. As a consequence, $a_{1}$ and $a_{2}$ represent the same element and thus functionality of $g^{\prime}$ implies that also $y_{1}$ and $y_{2}$ represent the same (concrete) element. To deal with such effects, we define an equivalence relation $\approx_{a}$ on abstract nodes and a second equivalence relation $\approx_{c}$ on concrete nodes.

Definition $4.3\left(\approx_{\mathrm{a}}\right.$ and $\approx_{\mathrm{c}}$ Relations). Let $S=(\mathbf{T}, \mathcal{P}, \prec, \sim)$ be a completion system for a concept $C$ and a key box $\mathcal{K}$ with $\mathbf{T}=\left(V_{a}, V_{c}, E, \mathcal{L}\right)$, and let $\approx$ be an equivalence relation on $V_{a}$. For each $R \in \mathrm{N}_{\mathrm{R}}$, a node $b \in V_{a}$ is an $R / \approx$-neighbor of a node $a \in V_{a}$ if there exists a node $c \in V_{a}$ such that $a \approx c$ and $b$ is an $R$-successor of $c$. Similarly, for each $g \in \mathrm{N}_{\mathrm{cF}}$, a node $x \in V_{c}$ is a $g / \approx$-neighbor of $a$ if there exists a node $c \in V_{a}$ such that $a \approx c$ and $x$ is a $g$-successor of $c$. For paths $u$, the notion of $u / \approx-n e i g h b o r$ is defined in the obvious way.

We define a sequence of equivalence relations $\approx_{\mathrm{a}}^{0} \subseteq \approx_{\mathrm{a}}^{1} \subseteq \cdots$ on $V_{a}$ as follows:

$$
\begin{aligned}
\approx_{\mathrm{a}}^{0}= & \left\{(a, a) \in V_{a}^{2}\right\} \cup \\
& \left\{(a, b) \in V_{a}^{2} \mid \text { there is an } N \in \mathrm{N}_{\mathrm{O}} \text { such that } N \in \mathcal{L}(a) \cap \mathcal{L}(b)\right\} \\
\approx_{\mathrm{a}}^{i+1}=\approx_{\mathrm{a}}^{i} \cup & \left\{(a, b) \in V_{a}^{2} \mid \text { there is a } c \in \mathrm{V}_{a} \text { and an } f \in \mathrm{N}_{\mathrm{aF}}\right. \text { such that } \\
& \left.a \text { and } b \text { are } f / \approx_{\mathrm{a}}^{i} \text {-neighbors of } c\right\} \cup \\
\left\{(a, b) \in V_{a}^{2} \mid\right. & \text { there is a }\left(u_{1}, \ldots, u_{n} \text { keyfor } C\right) \in \mathcal{K}, \\
& u_{i} / \approx_{\mathrm{a}}^{i} \text {-neighbors } x_{i} \text { of } a \text { for } 1 \leq i \leq n, \text { and } \\
& u_{i} / \approx_{\mathrm{a}}^{i} \text {-neighbors } y_{i} \text { of } b \text { for } 1 \leq i \leq n \\
& \text { such that } \left.C \in \mathcal{L}(a) \cap \mathcal{L}(b) \text { and } x_{i} \sim y_{i} \text { for } 1 \leq i \leq n\right\} .
\end{aligned}
$$

Finally, set $\approx_{\mathrm{a}}=\bigcup_{i \geq 0} \approx_{\mathrm{a}}^{i}$. Then define

$$
\begin{aligned}
\approx_{\mathrm{c}}=\sim \cup\left\{(x, y) \in V_{c}^{2} \mid\right. & \text { there is an } a \in V_{a} \text { and a } g \in \mathrm{N}_{\mathrm{cF}} \text { such that } \\
& \left.x \text { and } y \text { are } g / \approx_{\mathrm{a}} \text {-neighbors of } a\right\} .
\end{aligned}
$$


This definition reflects the above mentioned tight coupling between the concrete and abstract equalities: if the $\mathcal{D}$-tester finds (or guesses) that two concrete nodes are equal, the tableau algorithm may use this to deduce (via the computation of $\approx_{a}$ and $\approx_{c}$ ) even more equalities between concrete nodes.

Let $\mathcal{D}$ be a key-admissible concrete domain. To decide the satisfiability of an $\mathcal{A L C O K}(\mathcal{D})$ concept $C_{0}$ w.r.t. a Boolean key box $\mathcal{K}$ (both in NNF), the tableau algorithm is started with the initial completion tree

$$
\mathbf{T}_{C_{0}}=\left(\left\{a_{0}\right\}, \emptyset, \emptyset,\left\{a_{0} \mapsto\left\{C_{0}\right\}\right\}\right)
$$

in the initial completion system

$$
S_{C_{0}}=\left(\mathbf{T}_{C_{0}}, \mathcal{P}_{\emptyset}, \emptyset, \emptyset\right),
$$

where $\mathcal{P}_{\emptyset}$ maps each $P \in \Phi_{\mathcal{D}}$ occurring in $C_{0}$ to $\emptyset$. We now introduce an operation that is used by the completion rules to add new nodes to completion trees.

Definition 4.4 ("+" Operation). An abstract or concrete node is called fresh in a completion tree $\mathbf{T}$ if it does not appear in $\mathbf{T}$. Let $S=(\mathbf{T}, \mathcal{P}, \prec, \sim)$ be a completion system with $\mathbf{T}=\left(V_{a}, V_{c}, E, \mathcal{L}\right)$. We use the following notions:

- Let $a \in V_{a}, b \in \mathrm{O}_{\mathrm{a}}$ fresh in $\mathbf{T}$, and $R \in \mathrm{N}_{\mathrm{R}}$. We write $S+a R b$ to denote the completion system $S^{\prime}$ that can be obtained from $S$ by adding $b$ to $V_{a}$ and $(a, b)$ to $E$ and setting $\mathcal{L}(a, b)=R$ and $\mathcal{L}(b)=\emptyset$. Moreover, $b$ is inserted into $\prec$ such that $b \prec c$ implies $\operatorname{lev}_{\mathbf{T}}(b) \leq \operatorname{lev}_{\mathbf{T}}(c)$.

- Let $a \in V_{a}, x \in \mathrm{O}_{\mathrm{c}}$ fresh in $\mathbf{T}$ and $g \in \mathrm{N}_{\mathrm{cF}}$. We write $S+a g x$ to denote the completion system $S^{\prime}$ that can be obtained from $S$ by adding $x$ to $V_{c}$ and $(a, x)$ to $E$ and setting $\mathcal{L}(a, x)=g$.

When nesting the + operation, we omit brackets, writing, for example, $S+a R_{1} b+b R_{2} c$ for $\left(S+a R_{1} b\right)+b R_{2} c$. Let $u=f_{1} \cdots f_{n} g$ be a path. When $a \in V_{a}$ and $x \in \mathrm{O}_{c}$ is fresh in $\mathbf{T}$, we use $S+a u x$ to denote the completion system that is obtained from $S$ by taking distinct nodes $b_{1}, \ldots, b_{n} \in \mathrm{O}_{\mathrm{a}}$ which are fresh in $\mathbf{T}$ and setting

$$
S+a u x:=S+a f_{1} b_{1}+\cdots+b_{n-1} f_{n} b_{n}+b_{n} g x .
$$

Strictly speaking, the $S+a R b$ operation is non-deterministic since we did not specify how precisely the node $b$ is inserted into $\prec$. However, since this is don't care non-determinism, we will view the "+" operation as being deterministic.

The completion rules can be found in Figure 8. Note that the R $\sqcup$ and Rch rules are non-deterministic, i.e., they have more than one possible outcome (this is true don't know non-determinism). Some further remarks on the completion rules are in order: the upper five rules are well-known from existing tableau algorithms for $\mathcal{A L C}(\mathcal{D})$-concept satisfiability (see, e.g., Lutz, 2002a). Only the use of $R / \approx_{a}$-neighbors and $u / \approx_{a}$-neighbors in the rules $\mathrm{R} \forall, \mathrm{R} \exists$, and $\mathrm{R} \exists c$ deserves a comment. Take for example $\mathrm{R} \forall$ : intuitively, if we have $a \approx_{a} b$ for two abstract nodes $a$ and $b$ of the completion tree, then $a$ and $b$ describe the same domain element of the constructed model (and similarly for the $\approx_{c}$ relation on concrete 


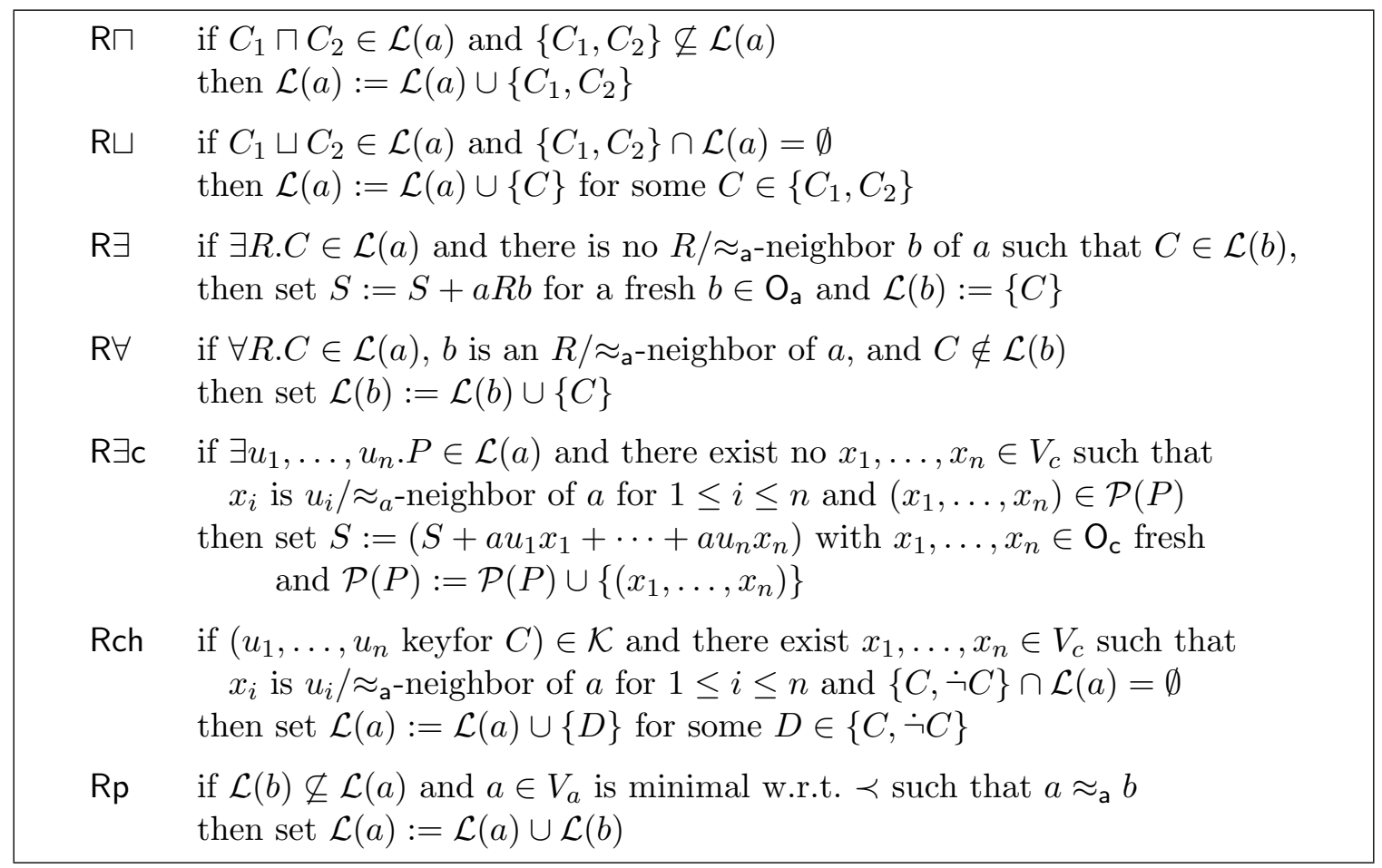

Figure 8: Completion rules for $\mathcal{A L C O K}(\mathcal{D})$.

nodes). Thus if $a \approx_{a} b$ and $c$ is an $R$-successor of $a$, then $c$ should also be an $R$-successor of $b$. However, since we want the completion tree to be a tree, we do not make the latter successorship explicit. To compensate for this, the $\mathrm{R} \forall$ rule talks about $R / \approx_{\mathrm{a}}$-neighbors rather than about $R$-successors.

The lower two rules are necessary for dealing with key boxes. The Rch rule is a so-called "choose rule" (Hollunder \& Baader, 1991; Horrocks et al., 2000): intuitively, it guesses whether or not an abstract node $a$ satisfies $C$ if there exists a key assertion $\left(u_{1}, \ldots, u_{n}\right.$ keyfor $\left.C\right) \in \mathcal{K}$ such that there are neighbors of $a$ for all the paths $u_{i}$. This is necessary since both possibilities may have ramifications: if $a$ satisfies $C$, then it must be taken into account in the construction of the relation $\approx_{a}$; if $a$ does not satisfy $C$, then we must deal with the consequences of it satisfying $\dot{\rightarrow} C$ (e.g. in case that $C$ is $\top$ ).

The $\mathrm{Rp}$ rule deals with equalities between abstract nodes as recorded by the $\approx_{a}$ relation: since $a \approx_{a} b$ means that $a$ and $b$ describe the same node in the constructed model, their node labels should be identical. It suffices, however, to choose one representative for each equivalence class of $\approx_{a}$ and make sure that this representative's node label contains the labels of all its $\approx_{a}$-equivalent nodes. As the representative, we use the node that is minimal w.r.t. the ordering $\prec$, which has been introduced solely for this reason. The Rp rule does the appropriate copying of node labels.

Let us now formalize what it means for a completion system to contain a contradiction.

Definition 4.5 (Clash). Let $S=(\mathbf{T}, \mathcal{P}, \prec, \sim)$ be a completion system for a concept $C$ and a key box $\mathcal{K}$ with $\mathbf{T}=\left(V_{a}, V_{c}, \prec, \sim\right)$. We say that the completion system $S$ is concrete 


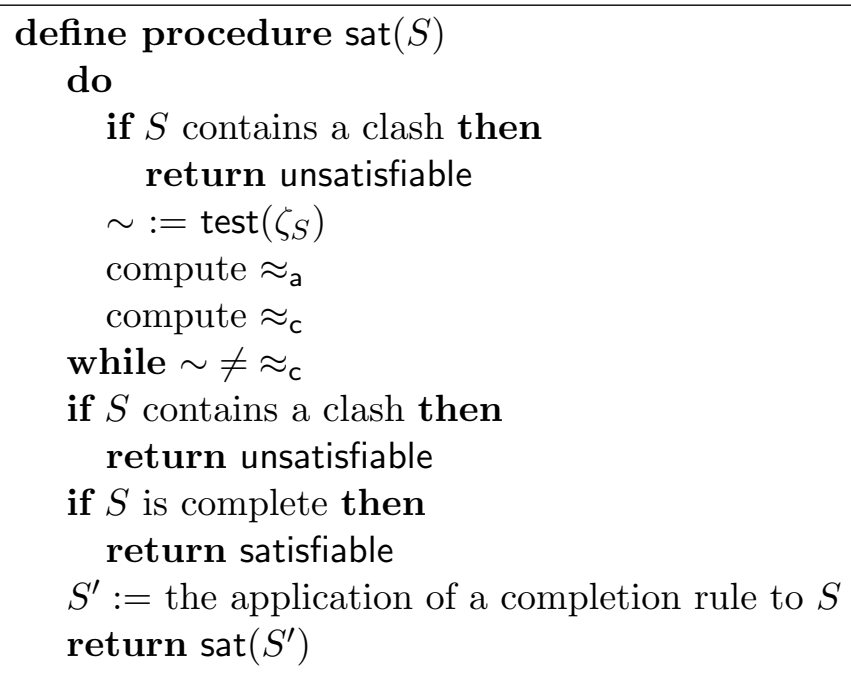

Figure 9: The $\mathcal{A L C O K}(\mathcal{D})$ tableau algorithm.

domain satisfiable iff the conjunction

$$
\zeta_{S}=\bigwedge_{P \text { used in } C} \bigwedge_{\left(x_{1}, \ldots, x_{n}\right) \in \mathcal{P}(P)} P\left(x_{1}, \ldots, x_{n}\right) \wedge \bigwedge_{x \approx_{c} y}=(x, y)
$$

is satisfiable. $S$ is said to contain a clash iff

1. there is an $a \in V_{a}$ and an $A \in \mathrm{N}_{\mathrm{C}}$ such that $\{A, \neg A\} \subseteq \mathcal{L}(a)$,

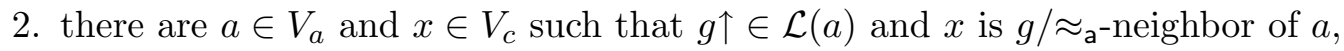

3. $S$ is not concrete domain satisfiable.

If $S$ does not contain a clash, $S$ is called clash-free. $S$ is called complete iff no completion rule is applicable to $S$.

The tableau algorithm is described in Figure 9 in pseudo-code notation. In this figure, test calls a $\mathcal{D}$-tester as specified in Definition 4.1. Let us say a few words about the while loop. There obviously exist close relationships between the relations $\sim$ and $\approx_{\mathrm{c}}$ and the predicate conjunction $\zeta_{S}$ :

- $\sim \subseteq \approx_{\mathrm{c}}$ (note that both $\approx_{\mathrm{a}}$ and $\approx_{\mathrm{c}}$ depend on $\sim$ and are thus recomputed in each step of the while loop);

- by definition of $\zeta_{S}$ and $\mathcal{D}$-tester, the result of test $\left(\zeta_{S}\right)$ yields a relation containing $\approx_{\mathrm{c}}$ (and thus also $\sim$ ).

Using these facts, one may check that, in each step of the while loop, new tuples are added to the $\sim$ relation, but none are deleted (see the proof of Lemma B.2 in the appendix). The while loop is needed because $(\mathrm{i}) \approx_{\mathrm{a}}$ is defined using $\sim,(\mathrm{ii}), \approx_{\mathrm{c}}$ is defined using $\approx_{\mathrm{a}}$, 
and (iii) new concrete equalities in $\approx_{c}$ may then imply even more concrete and/or abstract equalities, and so on.

A similar concrete-abstract interplay takes place in the course of several recursion steps: equalities between concrete nodes provided by the $\mathcal{D}$-tester may make new rules applicable (for example Rp and $\mathrm{R} \exists \mathrm{c}$ ) which changes $\mathcal{P}$ and thus also $\zeta_{S}$. This may subsequently lead to the detection of more equalities between concrete nodes by the $\mathcal{D}$-tester, and so on. These considerations show that, in the presence of keys, there exists a close interplay between the concrete domain reasoner and the tableau algorithm, which is not needed if keys are not present: without keys, it suffices to apply the concrete domain satisfiability check only once after the completion rules have been exhaustively applied (Baader \& Hanschke, 1991a).

The detailed proof of termination, soundness, and completeness together with a complexity analysis of the tableau algorithm defined in this section is given in Appendix B.

Theorem 4.1. Let $\mathcal{D}$ be a key-admissible concrete domain. If extended $\mathcal{D}$-satisfiability is in $\mathrm{NP}$, then $\mathcal{A} \mathcal{L C O K}(\mathcal{D})$-concept satisfiability w.r.t. Boolean key boxes is in NExPTIME.

We should note that, in the way it is presented here, the algorithm leaves considerable room for optimizations. One possible optimization concerns the "re-use" of $f$-successors (for abstract features $f$ ): for example, when applying the $\mathrm{R} \exists$ rule to a concept $\exists f . C \in \mathcal{L}(a)$, where $a$ already has an $f$-successor $b$, we could simply add $C$ to $\mathcal{L}(b)$ instead of adding a new $f$-successor $c$ and recording that $b \approx_{a} c$.

Another candidate for optimizations is the test function. Recall that this function takes a predicate conjunction $c$ with set of variables $V$ and non-deterministically returns a concrete equivalence, i.e., a relation $\sim$ such that there exists a solution $\delta$ for $c$ with $v_{i} \sim v_{j}$ iff $\delta\left(v_{i}\right)=\delta\left(v_{j}\right)$ (see Definition 4.1). It is not hard to devise an $\mathcal{A L C}(\mathcal{D})$-concept that forces completion systems to have exponentially many concrete nodes by slightly adapting wellknown $\mathcal{A L C}$-concepts that require models of exponential size (Halpern \& Moses, 1992). Hence, the size of input conjunctions $c$ to test can be exponential in the size of the input concept. Even for trivial $\mathcal{D}$-conjunctions

$$
c=\top_{\mathcal{D}}\left(v_{1}\right) \wedge \cdots \wedge \top_{\mathcal{D}}\left(v_{k}\right)
$$

we have an exponential number of distinct concrete equivalences $\sim$. Thus, the number of possible outcomes of a call to the test function may be double exponential in the size of the input concept. Considering the above example, a natural response to this problem is to require test to return only minimal concrete equivalences: intuitively, an equivalence is minimal if only those variables are equivalent whose equality is enforced by the conjunction.

More precisely, $\sim$ is called minimal if there exists no concrete equivalence $\sim^{\prime}$ such that $\left\{(x, y) \mid x \sim^{\prime} y\right\} \subset\{(x, y) \mid x \sim y\}$. We conjecture that restricting test in this way does not destroy the soundness and completeness of the tableau algorithm. However, although this definitely is a worthwhile optimization, it does not help to overcome the existence of doubly exponentially many outcomes of test in the worst case - at least not for all concrete domains $\mathcal{D}$ : consider the concrete domain $\mathrm{N}$ from Page 691 and conjunctions of the form

$$
c_{i}=<_{i}\left(v_{1}\right) \wedge \cdots \wedge<_{i}\left(v_{2 i}\right) .
$$

It is readily checked that, for each $i \geq 1$, the number of minimal concrete equivalences for $c_{i}$ is exponential in $i$. Moreover, it is not hard to devise a concept $C_{i}$ of size logarithmic 
in $i$ that leads to completion systems $S$ such that $\zeta_{S}=c_{i}$. Hence, there are still doubly exponentially many possible outcomes of the test function.

In the example just discussed, the exponential branching of test is clearly due to the discreteness of the natural numbers. Indeed, if we use a dense structure for defining concrete domains, it seems that the restriction to minimal concrete equivalences can have the desired effect, namely that the number of test's possible outcomes becomes polynomial in the size of its input and thus exponential in the size of the input concept. For example, consider the concrete domain $\mathrm{Q}$, which is defined as follows:

- $\Delta_{Q}$ is the set $\mathbb{Q}$ of rational numbers;

- $\Phi_{\mathrm{Q}}$ contains unary predicates $T_{\mathrm{Q}}$ and its negation $\perp_{\mathrm{Q}}$, unary predicates $=_{q}$ and $\neq_{q}$ for each $q \in \mathbb{Q}$, binary comparison predicates $\{<, \leq,=, \neq, \geq,>\}$, a ternary addition predicate + , and its negation $\mp$ (all with the obvious semantics).

It is readily checked that $Q$ is key-admissible (note that it provides a binary equality predicate) and thus falls into our framework. We conjecture that there exists only one minimal concrete equivalence for every Q-predicate conjunction $c$ : intuitively, it seems possible to (inductively) determine a relation $\sim$ on the set of variables $V$ used in $c$ such that (i) $x \sim y$ implies that $\delta(x)=\delta(y)$ for every solution $\delta$ for $c$ and (ii) there exists a solution $\delta$ for $c$ such that $v \nsim v^{\prime}$ implies $\delta(v) \neq \delta\left(v^{\prime}\right)$. Clearly, $\sim$ is a minimal concrete equivalence. Moreover, due to (i) it is the only one.

\subsection{A Tableau Algorithm for $\mathcal{S H O Q K}(\mathcal{D})$}

Although $\mathcal{A L C O K}(\mathcal{D})$ is a quite powerful DL, it lacks several expressive means that can be found in most state-of-the-art description logic systems such as FaCT and RACER (Horrocks, 1998; Horrocks et al., 2000; Haarslev \& Möller, 2001). In this section, we consider the very expressive description $\operatorname{logic} \mathcal{S H O} \mathcal{Q K}(\mathcal{D})$ which provides for concrete domains, key boxes, and nominals, but also for many other means of expressivity such as transitive roles, role hierarchies, qualifying number restrictions, and general TBoxes. Modulo some details, $\mathcal{S H O Q \mathcal { K }}(\mathcal{D})$ can be viewed as the extension of the DL $\mathcal{S H O} \mathcal{Q}(\mathcal{D})$ with key boxes. $\mathcal{S H O Q}(\mathcal{D})$ was proposed by Horrocks and Sattler (2001) (see also Pan \& Horrocks, 2002) as a tool for ontology reasoning in the context of the semantic web (Berners-Lee, Hendler, \& Lassila, 2001; Baader et al., 2002a).

One very important feature of $\mathcal{S H O} \mathcal{Q K}(\mathcal{D})$ are so-called TBoxes, i.e. concept equations ${ }^{5}$ of the form $C \doteq D$ that are used as a "background theory" in reasoning. Since it is wellknown that combining general TBoxes and the concrete domain constructor easily leads to undecidability (Baader \& Hanschke, 1992; Lutz, 2004), $\mathcal{S H O Q \mathcal { K }}(\mathcal{D})$ only offers a pathfree variant of the concrete domain constructor - i.e. only concrete features are admitted inside this constructor rather than paths of arbitrary length. This restriction indeed regains decidability (Haarslev et al., 2001; Horrocks \& Sattler, 2001). Path-freeness of the concrete domain constructor obviously renders abstract features unnecessary, and thus this syntactic type is not available in $\mathcal{S H O} \mathcal{Q K}(\mathcal{D})$.

5. Some TBox formalisms also allow for concept inclusions $C \sqsubseteq D$, but these can be re-written into equivalent equations, see Section 2.2.2.5 of (Baader et al., 2003). 


\subsubsection{The Description Logic $\mathcal{S H O} \mathcal{Q K}(\mathcal{D})$}

Let us now define $\mathcal{S H O} \mathcal{Q K}(\mathcal{D})$ in a formal way, starting with the syntax.

Definition 4.6 $(\mathcal{S H O} \mathcal{Q} \mathcal{K}(\mathcal{D})$ Syntax). A role axiom is either a role inclusion, which is of the form $R \sqsubseteq S$ with $R, S \in \mathrm{N}_{\mathrm{R}}$, or a transitivity axiom $\operatorname{Trans}(R)$ where $R \in \mathrm{N}_{\mathrm{R}}$. A role box $\mathcal{R}$ is a finite set of role axioms. Let $\stackrel{\Xi}{\underline{E}}$ be the reflexive-transitive closure of the role inclusions in $\mathcal{R}$. A role name $R$ is called simple if $S \stackrel{\mathscr{E}}{=} R$ implies $\operatorname{Trans}(S) \notin \mathcal{R}$ for all role names $S$. Let $\mathcal{D}$ be a concrete domain. The set of $\mathcal{S H O} \mathcal{Q K}(\mathcal{D})$-concepts is the smallest set such that

- every concept name and every nominal is a concept, and

- if $C$ and $D$ are concepts, $R$ is a role name, $S$ a simple role name, $n$ and $k$ are natural numbers, $g_{1}, \ldots, g_{n}$ are concrete features, and $P \in \Phi_{\mathcal{D}}$ is a predicate of arity $n$, then the following expressions are also concepts:

$$
\neg C, C \sqcap D, C \sqcup D, \exists R . C, \forall R . C,(\geqslant k S C),(\leqslant k S C), \exists g_{1}, \ldots, g_{n} . P \text {, and } g_{1} \uparrow .
$$

A concept equation is an expression $C \doteq D$ with $C$ and $D$ concepts. A TBox is a finite set of concept equations.

For $\mathcal{S H O} \mathcal{Q K}(\mathcal{D})$, we consider key boxes that differ in two aspects from the ones we considered for $\mathcal{A L C O K}(\mathcal{D})$ : in the following, we assume key boxes to be path-free, but we admit complex concepts to occur in key assertions. Note that abstract features and paths do not occur in the syntax of $\mathcal{S H O Q \mathcal { K }}(\mathcal{D})$ - as will become clear after the semantics has been defined, the former can be "simulated" by the more general number restrictions $(\leqslant n R C)$.

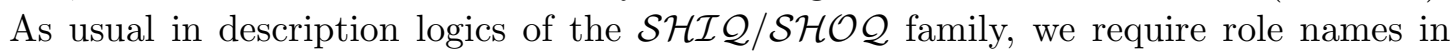
number restrictions to be simple since admitting arbitrary roles yields undecidability of reasoning (Horrocks et al., 2000; Horrocks \& Sattler, 2001). If the role box $\mathcal{R}$ is clear from the context, we will usually write $\operatorname{Trans}(R)$ instead of $\operatorname{Trans}(R) \in \mathcal{R}$. We now introduce the semantics of $\mathcal{S H O} \mathcal{Q K}(\mathcal{D})$ and the relevant reasoning problems.

Definition $4.7\left(\mathcal{S H O} \mathcal{H} \mathcal{K}(\mathcal{D})\right.$ Semantics). Interpretations $\mathcal{I}=\left(\Delta_{\mathcal{I}},{ }^{\mathcal{I}}\right)$ are defined as in Definition 2.3, where the function . ${ }^{\mathcal{I}}$ is extended to the novel $\mathcal{S H O} \mathcal{O} \mathcal{K}(\mathcal{D})$-concepts as follows:

$$
\begin{aligned}
& (\leqslant k R C)^{\mathcal{I}}:=\left\{d \in \Delta_{\mathcal{I}} \mid \sharp\left\{e \mid(d, e) \in R^{\mathcal{I}}\right\} \leq k\right\} \text { and } \\
& (\geqslant k R C)^{\mathcal{I}}:=\left\{d \in \Delta_{\mathcal{I}} \mid \sharp\left\{e \mid(d, e) \in R^{\mathcal{I}}\right\} \geq k\right\} .
\end{aligned}
$$

Let $\mathcal{I}$ be an interpretation. Then $\mathcal{I}$ satisfies a concept equation $C \doteq D$ if $C^{\mathcal{I}}=D^{\mathcal{I}}$. $\mathcal{I}$ is a model of a TBox $\mathcal{T}$ if $\mathcal{I}$ satisfies all concept equations in $\mathcal{T}$. Similarly, $\mathcal{I}$ satisfies a role inclusion $R \sqsubseteq S$ if $R^{\mathcal{I}} \subseteq S^{\mathcal{I}}$ and a transitivity axiom $\operatorname{Trans}(R)$ if $R^{\mathcal{I}}$ is a transitive relation. $\mathcal{I}$ is a model of a role box $\mathcal{R}$ if $\mathcal{I}$ satisfies all role inclusions and transitivity axioms in $\mathcal{R}$.

Let $\mathcal{T}$ be a TBox, $\mathcal{R}$ a role box, and $\mathcal{K}$ a key box. A concept $C$ is satisfiable w.r.t. $\mathcal{T}$, $\mathcal{R}$, and $\mathcal{K}$ iff $C, \mathcal{T}, \mathcal{R}$, and $\mathcal{K}$ have a common model. $C$ is subsumed by a concept $D$ w.r.t. $\mathcal{T}$, $\mathcal{R}$, and $\mathcal{K}$ (written $C \sqsubseteq \mathcal{T}, \mathcal{R}, \mathcal{K} D$ ) iff $C^{\mathcal{I}} \subseteq D^{\mathcal{I}}$ for all common models $\mathcal{I}$ of $\mathcal{T}, \mathcal{R}$, and $\mathcal{K}$. 
Note that, due to the requirement that role names used inside number restrictions should be simple, existential and universal value restrictions are not just syntactic sugar: in contrast to number restrictions, they can be used on all roles.

It is well-known that, in many expressive description logics, reasoning with TBoxes can be reduced to reasoning without them (Schild, 1991; Horrocks \& Sattler, 2001): for $\mathcal{S H O Q \mathcal { K }}(\mathcal{D})$, a concept $C$ is satisfiable w.r.t. $\mathcal{T}, \mathcal{R}$, and $\mathcal{K}$ iff the concept

$$
\exists R . C \sqcap \forall R .\left(\prod_{D \stackrel{\equiv}{=} E \in \mathcal{T}} D \leftrightarrow E\right) \sqcap \prod_{\substack{\text { nominal } N \text { used } \\ \text { in } C, \mathcal{T}, \text { or } \mathcal{K}}}(\exists R . N)
$$

is satisfiable w.r.t. to $\mathcal{R}^{\prime}, \mathcal{K}$, and the empty TBox, where $R$ is a fresh role not appearing in $C, \mathcal{R}$, and $\mathcal{T}$, and

$$
\begin{aligned}
\mathcal{R}^{\prime}:=\mathcal{R} \cup\{\operatorname{Trans}(R)\} & \cup \backslash S \subseteq R\} . \\
& \text { role name } S \text { used } \\
& \text { in } C, \mathcal{T}, \mathcal{R}, \text { or } \mathcal{K}
\end{aligned}
$$

Since subsumption can be reduced to satisfiability as described in Section 2, in the following we will only consider satisfiability of concepts w.r.t. role boxes and key boxes, but without TBoxes. We will also generally assume role boxes $\mathcal{R}$ to be acyclic, i.e. to satisfy the following condition: for each role name $R$, there are no role names $R_{1}, \ldots, R_{k}$ such that $R=R_{1}=R_{k}$ and $R_{i} \sqsubseteq R_{i+1} \in \mathcal{R}$ for $1 \leq i<k$. It is not hard to see that this is not a restriction since cycles can be eliminated: if $R_{1}, \ldots, R_{k}$ is a cycle in $\mathcal{R}$, then we have $R_{1}^{\mathcal{I}}=\cdots=R_{k}^{\mathcal{I}}$ for all interpretations $\mathcal{I}$. Thus we can simply remove the cycle from $\mathcal{R}$ and replace every occurrence of $R_{2}, \ldots, R_{k}$ in $C, \mathcal{R}$, and $\mathcal{K}$ with $R_{1}$, and add $\operatorname{Trans}\left(R_{1}\right)$ if, before the cycle elimination, we had $\operatorname{Trans}\left(R_{i}\right)$ for some $i$ with $1 \leq i \leq n$.

Before we turn our attention to the construction of a tableau algorithm for $\mathcal{S H O Q K}(\mathcal{D})$, let us comment on a few minor differences between $\mathcal{S H O Q K}(\mathcal{D})$ as introduced here and the original version of $\mathcal{S H O Q Q}(\mathcal{D})$ as described in (Horrocks \& Sattler, 2001). The main difference is that our logic, like the extensions investigated in (Haarslev et al., 2001; Pan \& Horrocks, 2002), allows $n$-ary predicates while Horrocks and Sattler restrict themselves to unary predicates. Moreover, $\mathcal{S H O Q}(\mathcal{D})$ as introduced in (Horrocks \& Sattler, 2001) uses concrete roles rather than concrete features, the difference being that concrete roles are not necessarily functional. Due to this non-functionality, the original $\mathcal{S H O Q Q}(\mathcal{D})$ admits two variants $\exists T . P$ and $\forall T$.P of the concrete domain constructor (where $T$ is a concrete role and $P$ a unary predicate). In $\mathcal{S H O} \mathcal{Q K}(\mathcal{D})$, we can simulate the universal variant by writing $\exists g . P \sqcup g \uparrow$ since concrete features $g$ are interpreted as partial functions and, in contrast to Horrocks and Sattler, we have the undefinedness constructor $g \uparrow$ available. Except for the $n$-ary predicates which provide important additional expressivity, we view these deviations as minor ones since it is easy to see that they do not affect decidability and complexity of reasoning.

\subsubsection{A Tableau Algorithm for $\mathcal{S H O} \mathcal{Q K}(\mathcal{D})$}

The basic intuitions of the $\mathcal{S H O} \mathcal{Q K}(\mathcal{D})$ tableau algorithm are similar to the $\mathcal{A} \mathcal{L C O K}(\mathcal{D})$ algorithm, with one exception: to deal with the various expressive means of $\mathcal{S H O} \mathcal{Q K}(\mathcal{D})$, 


$$
\begin{aligned}
& \neg(\geqslant n R C) \quad \rightsquigarrow \quad(\leqslant(n-1) R C) \text { if } n \geq 1 \\
& \neg(\geqslant 0 R C) \quad \rightsquigarrow \quad \perp \\
& \neg(\leqslant n R C) \quad \rightsquigarrow(\geqslant(n+1) R C)
\end{aligned}
$$

Figure 10: The $\mathcal{S H O} \mathcal{Q K}(\mathcal{D})$ NNF rewrite rules.

it is convenient to introduce a certain abstraction of models, so-called tableaux. The main difference between tableaux and models is that, in tableaux, roles declared to be transitive are not necessarily described by transitive relations. We show that there exists a tableau for a given concept and key box if and only if they have a common model. The aim of the $\mathcal{S H O Q K}(\mathcal{D})$ algorithm is then to construct a tableau for its input rather than trying to construct a model. To do this, the algorithm employs completion forests as its underlying data structure.

We first introduce tableaux. Let us start by discussing some preliminaries. As for $\mathcal{A L C O K}(\mathcal{D})$, we assume all concepts and key boxes to be in NNF, i.e. negation occurs only in front of concept names and nominals. We again use $\rightarrow C$ to denote the NNF of $\neg C$. The additional NNF rewrite rules for $\mathcal{S H O} \mathcal{Q K}(\mathcal{D})$ can be found in Figure 10 and complete those given for $\mathcal{A L C O K}(\mathcal{D})$ in Figure 7 .

For a concept $D$, role box $\mathcal{R}$, and key box $\mathcal{K}$, we define

$$
\begin{aligned}
\operatorname{cl}(D, \mathcal{K}) & :=\operatorname{sub}(D) \cup \operatorname{sub}(\operatorname{con}(\mathcal{K})) \cup\{\dot{\neg} C \mid C \in \operatorname{sub}(D) \cup \operatorname{sub}(\operatorname{con}(\mathcal{K}))\} \\
\operatorname{cl}(D, \mathcal{R}, \mathcal{K}) & :=\operatorname{cl}(D, \mathcal{K}) \cup\{\forall R . C \mid R \underline{\underline{\Psi}} S \text { and } \forall S . C \in \operatorname{cl}(D, \mathcal{K})\} .
\end{aligned}
$$

Obviously, the cardinality of $\operatorname{cl}(D, \mathcal{R}, \mathcal{K})$ is linear in the size of $D, \mathcal{R}$, and $\mathcal{K}$. In what follows, we write $\mathrm{N}_{\mathrm{R}}^{D, \mathcal{R}, \mathcal{K}}$ to denote the set of role names occurring in $D, \mathcal{R}$, or $\mathcal{K}$, and $\mathrm{N}_{\mathrm{cF}}^{D, \mathcal{K}}$ to denote the sets of concrete features occurring in $D$ or $\mathcal{K}$. We are now ready to define tableaux.

Definition 4.8 (Tableau). Let $D$ be a $\mathcal{S H O} \mathcal{Q K}(\mathcal{D})$-concept in NNF, $\mathcal{R}$ a role box, and $\mathcal{K}$ a path-free key box in NNF. A tableau $T$ for $D$ w.r.t. $\mathcal{R}$ and $\mathcal{K}$ is a tuple $\left(\mathbf{S}_{a}, \mathbf{S}_{c}, \mathcal{L}, E, e, \mathcal{P}\right)$ such that

- $\mathbf{S}_{a}, \mathbf{S}_{c}$ are sets of abstract and concrete individuals,

- $\mathcal{L}: \mathbf{S}_{a} \rightarrow 2^{\mathrm{cl}(D, \mathcal{R}, \mathcal{K})}$ maps each abstract individual to a subset of $\operatorname{cl}(D, \mathcal{R}, \mathcal{K})$,

- $E: \mathbf{S}_{a} \times \mathbf{S}_{a} \rightarrow 2^{\mathrm{N}_{\mathrm{R}}^{D, \mathcal{R}, \mathcal{K}}}$ maps pairs of abstract individuals to sets of roles,

- $e: \mathbf{S}_{a} \times \mathrm{N}_{\mathrm{cF}}^{D, \mathcal{K}} \rightarrow \mathbf{S}_{c}$ maps pairs of abstract individuals and concrete features to concrete individuals,

- $\mathcal{P}$ maps each $n$-ary concrete predicate in $\operatorname{cl}(D, \mathcal{R}, \mathcal{K})$ to a set of $n$-tuples over $\mathbf{S}_{c}$,

- there is an abstract individual $s_{0} \in \mathbf{S}_{a}$ such that $D \in \mathcal{L}\left(s_{0}\right)$, and

for all $s, t \in \mathbf{S}_{a}, C, C_{1}, C_{2} \in \mathrm{cl}(D, \mathcal{R}, \mathcal{K}), R, S \in \mathrm{N}_{\mathrm{R}}^{D, \mathcal{R}, \mathcal{K}}$, and for

$$
S^{T}(s, C):=\left\{t \in \mathbf{S}_{a} \mid S \in E(s, t) \text { and } C \in \mathcal{L}(t)\right\}
$$

it is the case that: 
(T1) if $C \in \mathcal{L}(s)$, then $\dot{\rightarrow} C \notin \mathcal{L}(s)$,

(T2) if $C_{1} \sqcap C_{2} \in \mathcal{L}(s)$, then $C_{1} \in \mathcal{L}(s)$ and $C_{2} \in \mathcal{L}(s)$,

(T3) if $C_{1} \sqcup C_{2} \in \mathcal{L}(s)$, then $C_{1} \in \mathcal{L}(s)$ or $C_{2} \in \mathcal{L}(s)$,

(T4) if $R \in E(s, t)$ and $R \stackrel{E}{=} S$, then $S \in E(s, t)$,

(T5) if $\forall R . C \in \mathcal{L}(s)$ and $R \in E(s, t)$, then $C \in \mathcal{L}(t)$,

(T6) if $\exists R . C \in \mathcal{L}(s)$, then there is some $t \in \mathbf{S}_{a}$ such that $R \in E(s, t)$ and $C \in \mathcal{L}(t)$,

(T7) if $\forall S . C \in \mathcal{L}(s)$ and $R \in E(s, t)$ for some $R \stackrel{\circledast}{=}$ with $\operatorname{Trans}(R)$, then $\forall R . C \in \mathcal{L}(t)$,

(T8) if $(\geqslant n S C) \in \mathcal{L}(s)$, then $\sharp S^{T}(s, C) \geqslant n$,

(T9) if $(\leqslant n S C) \in \mathcal{L}(s)$, then $\sharp S^{T}(s, C) \leqslant n$,

(T10) if either $(\leqslant n S C) \in \mathcal{L}(s)$ and $S \in E(s, t)$ or $\left(g_{1}, \ldots, g_{n}\right.$ keyfor $\left.C\right) \in \mathcal{K}$ and $e\left(t, g_{i}\right)$ is defined for all $1 \leq i \leq n$, then $\{C, \dot{\neg} C\} \cap \mathcal{L}(t) \neq \emptyset$,

(T11) if $N \in \mathcal{L}(s) \cap \mathcal{L}(t)$, then $s=t$,

(T12) if $\exists g_{1}, \ldots, g_{n} . P \in \mathcal{L}(s)$, then there are $x_{1}, \ldots, x_{n} \in \mathbf{S}_{c}$ with $e\left(s, g_{i}\right)=x_{i}$ and $\left(x_{1}, \ldots, x_{n}\right) \in \mathcal{P}(P)$,

(T13) $\bigwedge_{P}$ used in $D, \mathcal{K} \bigwedge_{\left(x_{1}, \ldots, x_{n}\right) \in \mathcal{P}(P)} P\left(x_{1}, \ldots, x_{n}\right) \wedge \bigwedge_{x \neq y} x \neq y$ is satisfiable,

(T14) if $\left(g_{1}, \ldots, g_{n}\right.$ keyfor $\left.C\right) \in \mathcal{K}, C \in \mathcal{L}(s) \cap \mathcal{L}(t)$, and $e\left(s, g_{i}\right)=e\left(t, g_{i}\right)$ for all $1 \leq i \leq n$, then $s=t$,

(T15) if $g \uparrow \in \mathcal{L}(s)$, then $e(s, g)$ is undefined.

Note that the predicate conjunction in (T13) uses a binary inequality predicate. In general, we do not require the concrete domain $\mathcal{D}$ to be equipped with such a predicate and thus this predicate conjunction is not necessarily a $\mathcal{D}$-conjunction. However, it is nevertheless "safe" to use (T13) in the given form since tableaux are only used in proofs and we do not need a concrete domain reasoner that is capable of deciding the satisfiability of this conjunction. The following lemma, whose proof is provided in Appendix C, shows that our definition of tableaux provides an adequate abstraction of models.

Lemma 4.2. Let $D$ be a $\mathcal{S H O Q \mathcal { K }}(\mathcal{D})$-concept in $N N F, \mathcal{R}$ a role box, and $\mathcal{K}$ a key box in $N N F$. Then $D$ is satisfiable w.r.t. $\mathcal{R}$ and $\mathcal{K}$ iff $D$ has a tableau w.r.t. $\mathcal{R}$ and $\mathcal{K}$.

Given Lemma 4.2, in order to decide satisfiability of $\mathcal{S H O} \mathcal{Q K}(\mathcal{D})$-concepts w.r.t. role and key boxes, we may use a (tableau) algorithm that tries to construct a tableau for the input. In the following, we will describe such an algorithm in detail.

As in the previous section, the algorithm works on completion systems. However, in the case of $\mathcal{S H O Q K}(\mathcal{D})$ the core component of completion systems is a completion forest rather than a completion tree. The reason for this is that some completion rules remove nodes and edges from the completion system and in this way can disconnect one tree into two subtrees. 
Definition 4.9 (Completion System). Let $D$ be a $\mathcal{S H O} \mathcal{Q K}(\mathcal{D})$-concept in NNF, $\mathcal{R}$ a role box, and $\mathcal{K}$ a path-free key box in NNF. For each concept $(\geqslant n R C) \in \operatorname{cl}(D, \mathcal{R}, \mathcal{K})$ and $1 \leq i \leq n$, we reserve a concept name $A_{i}^{n R C}$ not appearing in $\operatorname{cl}(D, \mathcal{R}, \mathcal{K})$ and define an extended closure

$$
\mathrm{cl}^{+}(D, \mathcal{R}, \mathcal{K}):=\mathrm{cl}(D, \mathcal{R}, \mathcal{K}) \cup\left\{A_{1}^{n R c}, \ldots, A_{n}^{n R c} \mid(\geqslant n R C) \in \operatorname{cl}(D, \mathcal{R}, \mathcal{K})\right\} .
$$

Let $\mathrm{O}_{a}$ and $\mathrm{O}_{c}$ be disjoint and countably infinite sets of abstract and concrete nodes. A completion forest for $D, \mathcal{R}$, and $\mathcal{K}$ is a finite forest $\mathbf{F}=\left(V_{a}, V_{c}, E, \mathcal{L}\right)$ with nodes $V_{a} \cup V_{c}$ such that $V_{a} \subseteq \mathrm{O}_{\mathrm{a}}, V_{c} \subseteq \mathrm{O}_{\mathrm{c}}$, and all nodes from $V_{c}$ are leaves. The forest is labelled as follows:

- each node $a \in V_{a}$ is labelled with a subset $\mathcal{L}(a)$ of $\mathrm{cl}^{+}(D, \mathcal{R}, \mathcal{K})$,

- each edge $(a, b) \in E$ with $a, b \in V_{a}$ is labeled with a non-empty set of role names $\mathcal{L}(a, b)$ occurring in $D, \mathcal{R}$, or $\mathcal{K}$, and

- each edge $(a, x) \in E$ with $a \in V_{a}$ and $x \in V_{c}$ is labeled with a concrete feature $\mathcal{L}(a, x)$ occurring in $D, \mathcal{R}$, or $\mathcal{K}$.

A completion system for $D, \mathcal{R}$, and $\mathcal{K}$ is a tuple $S=\left(\mathbf{F}, \mathcal{P}, \sim_{c}, \prec\right)$ such that

- $\mathbf{F}=\left(V_{a}, V_{c}, E, \mathcal{L}\right)$ is a completion forest for $D, \mathcal{R}$, and $\mathcal{K}$,

- $\mathcal{P}$ maps each $n$-ary concrete predicate in $\operatorname{cl}(D, \mathcal{R}, \mathcal{K})$ to a set of $n$-tuples in $V_{c}$,

- $\sim_{\mathrm{c}}$ is an equivalence relation on $V_{c}$, and

- $\prec$ is a linear ordering on $V_{a}$.

A node $t \in V_{a}$ is called an $R$-successor of a node $s \in V_{a}$ if, for some $R^{\prime}$ with $R^{\prime} \stackrel{\Xi}{=} R$, we have $R^{\prime} \in \mathcal{L}(s, t)$. A node $x \in V_{c}$ is called a $g$-successor of a node $s \in V_{a}$ if $\mathcal{L}(s, x)=g$. Finally, we write $s \neq t$ if $s$ and $t$ are $R$-successors of the same node and there is some $A_{i}^{n R C} \in \mathcal{L}(s)$ and $A_{j}^{n R C} \in \mathcal{L}(t)$ with $i \neq j$.

Some remarks are in order here. Firstly, in contrast to the $\mathcal{A L C O K}(\mathcal{D})$ case, the relation $\prec$ is no longer required to respect the level of a node. This is due to the fact that (a) we have to enforce termination artificially and the mentioned property of $\prec$ was used to ensure "automatic" termination, and (b) the level of a node might change since a node might become a root node because some completion rules will remove nodes and edges.

Secondly, the relation $\sim_{c}$ will be returned by a $\mathcal{D}$-tester, and is used to compute a relation $\approx_{a}$ which is then used by the tableau algorithm. However, we do not need to compute the relation $\approx_{c}$ from $\approx_{a}$ as in the $\mathcal{A L C O K}(\mathcal{D})$ case since all concepts and key boxes are assumed to be path-free.

Thirdly, the new concept names $A_{i}^{n R C}$ will be used to ensure that successors of a node $x$ generated for some $(\geqslant n R C) \in \mathcal{L}(x)$ will not be merged later due to a concept ( $\leqslant$ $\left.n^{\prime} R C^{\prime}\right) \in \mathcal{L}(x)$ : each generated successor is labelled with a different concept $A_{i}^{n R C}$; since merging two nodes means unifying their node labels, it then suffices to disallow the 
occurrence of distinct concepts $A_{i}^{n R C}$ in the same node label through a suitable definition of "clash".

Since $\mathcal{S H O} \mathcal{Q K}(\mathcal{D})$ provides for transitive roles, we need some cycle-detection mechanism in order to guarantee termination of our algorithm: roughly speaking, if we encounter a node which is "similar" to an already existing one, then this node does not need to be further explored. Speaking in terms of (Horrocks et al., 2000; Baader \& Sattler, 2000), we employ a mechanism called subset blocking.

Definition 4.10 (Blocked). Let $\preceq$ be the reflexive closure of $\prec$. A node $t \in V_{a}$ is blocked by a node $s \in V_{a}$ if $\mathcal{L}(t) \subseteq \mathcal{L}(s)$, and $s \preceq s^{\prime}$, for all $s^{\prime}$ with $\mathcal{L}(t) \subseteq \mathcal{L}\left(s^{\prime}\right)$.

Note that, unlike to what is done, e.g., in (Horrocks et al., 2000), the blocking node is not necessarily an ancestor of the blocked node, but can be anywhere in the forest. It may even be that blocked nodes have unblocked successors. This modification is used later to obtain a NExpTime upper bound.

To decide the satisfiability of an $\mathcal{A L C O K}(\mathcal{D})$-concept $D$ w.r.t. a role box $\mathcal{R}$ and a pathfree key box $\mathcal{K}$ (where $D$ and $\mathcal{K}$ are in NNF), the tableau algorithm is started with the initial completion system

$$
\begin{aligned}
S_{D}= & \left(\mathbf{F}_{D}, \mathcal{P}_{\emptyset}, \emptyset, \emptyset\right), \text { where } \\
\mathbf{F}_{D}= & \left(\left\{s_{0}\right\}, \emptyset, \emptyset,\left\{s_{0} \mapsto\{D\}\right\}\right) \text { and } \\
& \text { maps each } P \in \Phi_{\mathcal{D}} \text { occurring in } D \text { and } \mathcal{K} \text { to } \emptyset .
\end{aligned}
$$

Then the algorithm repeatedly applies completion rules. Before the actual rules are given, we introduce some new notions: firstly, we define the equivalence relation $\approx_{a}$ over $V_{a}$ as follows: $s \approx_{\mathrm{a}} t$ if one of the following conditions is satisfied:

- $N \in \mathcal{L}(s) \cap \mathcal{L}(t)$ for some nominal $N$ or

- $\left(g_{1} \ldots, g_{n}\right.$ keyfor $\left.C\right) \in \mathcal{K}, C \in \mathcal{L}(s) \cap \mathcal{L}(t)$, and there are $x_{i}, y_{i}$ such that $g_{i} \in E\left(s, x_{i}\right) \cap$ $E\left(t, y_{i}\right)$ and $x_{i} \sim_{\mathrm{c}} y_{i}$ for $1 \leq i \leq n$.

Intuitively, two abstract nodes related via the $\approx_{a}$ relation describe the same individual in a tableau.

Secondly, we use the following abbreviations in the formulation of the rules (written in italic):

- To remove an abstract node $s$ and all its incoming and outgoing edges, remove $s$ from $V_{a}$ and each $(s, t)$ and $(t, s)$ from $E$ for all $t \in V_{a} \cup V_{c}$.

- Adding a g-successor of an abstract node $s$ means doing nothing if there exists a $g$-successor $x \in V_{c}$ of $s$ and, otherwise, adding $E(s, x)=g$ for some $x \in \mathrm{O}_{\mathrm{c}}$ that does not yet occur in the completion forest.

- To update the relation $\sim_{\mathrm{c}}$, a $\mathcal{D}$-tester is asked to decide the satisfiability of the $\mathcal{D}$ conjunction

$$
\zeta_{S}:=\bigwedge_{\substack{P \text { used in } D, \mathcal{K} \\\left(x_{1}, \ldots, x_{n}\right) \in \mathcal{P}(P)}} P\left(x_{1}, \ldots, x_{n}\right) \wedge \bigwedge_{x \sim_{c} y} x=y
$$

and returns, in case that this conjunction is satisfiable, an "updated" concrete equivalence $\sim_{c}$ as defined in Definition 4.1. 
Concerning the predicate conjunction used in updates, recall that we can w.l.o.g. assume the concrete domain to contain an equality predicate as discussed after Definition 4.1.

The completion rules are given in Figure 11. We generally assume that new nodes $x$ are introduced into the completion forest such that $y \prec x$ for all already existing nodes $y$. Before further describing the tableau algorithm, we comment on the completion rules. The rules $\mathrm{R} \sqcup, \mathrm{R} \leqslant, \mathrm{R} \exists \mathrm{c}$, and Rch are non-deterministic, i.e., their application has more than one possible outcome. For the $\mathrm{R} \exists \mathrm{c}$ rule, this is due to the update operation performed on $\sim_{\mathrm{c}}$ using the $\mathcal{D}$-tester: as discussed at the end of Section 4.1, computing a concrete equivalence for a given $\mathcal{D}$-conjunction may result in a high degree of non-determinism. Please note that, in contrast to $\mathcal{A L C O K}(\mathcal{D})$, we now only need to call the $\mathcal{D}$-tester in this rule - and not after each rule application.

Next, the $R \approx_{a}$ rule takes care of abstract nodes related via $\approx_{a}$. Since all the nodes from $\mathrm{a} \approx_{\mathrm{a}}$-equivalence class denote the same individual, we choose only one representative whose node label contains the labels of all other nodes in the class. This representative simply is the $\prec$-minimal node of the equivalence class and the $\mathrm{R} \approx_{a}$ rule performs the appropriate copying of node labels.

The $\mathrm{R} \leqslant$ rule is the only rule to remove nodes and edges: it removes a surplus $R$-successor $t$ of a node $s$ with $(\leqslant n R C) \in \mathcal{L}(s)$. Since the subtree below $t$ is not removed, $t$ 's successors are new, additional root nodes. This behavior is the reason why we work on a completion forest.

As in the $\mathcal{A L C O K}(\mathcal{D})$ case, the tableau algorithm stops applying rules if it finds an obvious contradiction, a "clash", or if no more completion rules are applicable.

Definition 4.11 (Clash). Let $S=\left(\mathbf{F}, \mathcal{P}, \sim_{c}, \prec\right)$ be a completion system for $D, \mathcal{R}$ and $\mathcal{K}$, and $\mathbf{F}=\left(V_{a}, V_{c}, E, \mathcal{L}\right)$. Then $S$ is said to contain a clash if one of the following conditions applies:

(C1) for some concept name $A \in \mathrm{N}_{\mathrm{C}}$ and some node $s \in V_{a},\{A, \neg A\} \subseteq \mathcal{L}(s)$;

(C2) the $\mathcal{D}$-conjunction $\zeta_{S}$ defined above is not satisfiable;

(C3) $s \neq s$ for some $s \in V_{a}$;

(C4) for some $s \in V_{a}$ and $g \in \mathrm{N}_{\mathrm{cF}}$, we have $g \uparrow \in \mathcal{L}(s)$ and $s$ has a $g$-successor.

A completion system not containing a clash is called clash-free. The completion system is complete if none of the completion rules is applicable.

Due to the simplicity of the algorithm, we refrain from describing it in pseudo-code notation: the algorithm starts with the initial completion system and then repeatedly applies the completion rules, checking for clashes after each rule application. If a clash is detected, it returns unsatisfiable. If a complete and clash-free completion system is found, then the algorithm returns satisfiable. Note that, since some of the completion rules are non-deterministic, the algorithm is also non-deterministic.

Details of the proof of termination, soundness, and completeness are given in Appendix C. Unfortunately, we have to leave the complexity of the algorithm as an open problem: it is not hard to prove that it runs in double exponential time, but it is not clear whether exponential time also suffices. However, we can still use the algorithm to obtain a 
$\mathrm{R} \sqcap \quad$ if $C_{1} \sqcap C_{2} \in \mathcal{L}(s), s$ is not blocked, and $\left\{C_{1}, C_{2}\right\} \nsubseteq \mathcal{L}(s)$, then $\mathcal{L}(s):=\mathcal{L}(s) \cup\left\{C_{1}, C_{2}\right\}$

$\mathrm{R} \sqcup \quad$ if $C_{1} \sqcup C_{2} \in \mathcal{L}(s), s$ is not blocked, and $\left\{C_{1}, C_{2}\right\} \cap \mathcal{L}(s)=\emptyset$, then $\mathcal{L}(s):=\mathcal{L}(s) \cup\{C\}$ for some $C \in\left\{C_{1}, C_{2}\right\}$

$\mathrm{R} \exists \quad$ if $\exists R . C \in \mathcal{L}(s), s$ is not blocked, and $s$ has no $R$-successor $t$ with $C \in \mathcal{L}(t)$ then create a new node $t$ such that $t^{\prime} \prec t$ for all $t^{\prime} \in V_{a}$ and set $E(s, t):=\{R\}$ and $\mathcal{L}(t):=\{C\}$

$\mathrm{R} \geqslant \quad$ if $(\geqslant n S C) \in \mathcal{L}(s), s$ is not blocked, and there are no $n S$-successors $t_{1}, \ldots, t_{n}$ of $s$ with $C \in \mathcal{L}\left(t_{i}\right)$ and $t_{i} \neq t_{j}$ for $1 \leq i<j \leq n$, then create $n$ new nodes $t_{1}, \ldots, t_{n}$ s.t. $t^{\prime} \prec t_{i}$ for $1 \leq i \leq n$ and all $t^{\prime} \in V_{a}$, and set $E\left(s, t_{i}\right):=\{S\}$ and $\mathcal{L}\left(t_{i}\right):=\left\{C, A_{i}^{n S C}\right\}$ for $1 \leq i \leq n$

$\mathrm{R} \leqslant \quad$ if $(\leqslant n S C) \in \mathcal{L}(s), s$ is not blocked, $s$ has $n+1 S$-successors $t_{0}, \ldots, t_{n}$ with $C \in \mathcal{L}\left(t_{i}\right)$ for $0 \leq i \leq n$,

then choose $i, j$ such that $t_{i} \prec t_{j}$, set $\mathcal{L}\left(t_{i}\right):=\mathcal{L}\left(t_{i}\right) \cup \mathcal{L}\left(t_{j}\right)$, $\mathcal{L}\left(s, t_{i}\right):=\mathcal{L}\left(s, t_{i}\right) \cup \mathcal{L}\left(s, t_{j}\right)$, and remove $t_{j}$ and all its incoming and outgoing edges

$\mathrm{R} \exists \mathrm{c} \quad$ if $\exists g_{1}, \ldots, g_{n} . P \in \mathcal{L}(s), s$ is not blocked, and

there are no $g_{i}$-successors $x_{i}$ with $\left(x_{1}, \ldots, x_{n}\right) \in \mathcal{P}(P)$

then add a $g_{i}$-successor of $s$ for each $1 \leq i \leq n$,

for $y_{i}$ the $g_{i}$-successor of $s$, add $\left(y_{1}, \ldots, y_{n}\right)$ to $\mathcal{P}(P)$, and update $\sim_{c}$

$\mathrm{R} \forall \quad$ if $\forall R . C \in \mathcal{L}(s), s$ is not blocked, and

there is an $R$-successor $t$ of $s$ with $C \notin \mathcal{L}(t)$,

then $\mathcal{L}(t):=\mathcal{L}(t) \cup\{C\}$

$\mathrm{R} \forall_{+} \quad$ if $\forall S . C \in \mathcal{L}(s), s$ is not blocked, there is some $R$ with

$\operatorname{Trans}(R)$ and $R \underline{E} S$, and an $R$-successor $t$ of $s$ with $\forall R . C \notin \mathcal{L}(t)$, then $\mathcal{L}(t):=\mathcal{L}(t) \cup\{\forall R . C\}$

Rch if $s$ is an $S$-successor of $s^{\prime}$ and $(\leqslant n S C) \in \mathcal{L}\left(s^{\prime}\right)$ or $s$ has $g_{i}$-successors $x_{i}$ for all $1 \leq i \leq n$ and $\left(g_{1}, \ldots g_{n}\right.$ keyfor $\left.C\right) \in \mathcal{K}$ and $s$ is not blocked and $\{C, \dot{\rightarrow} C\} \cap \mathcal{L}(s)=\emptyset$, then $\mathcal{L}(s):=\mathcal{L}(s) \cup\{E\}$ for some $E \in\{C, \dot{\neg} C\}$

$\mathrm{R} \approx_{\mathrm{a}} \quad$ if $s \approx_{\mathrm{a}} t, \mathcal{L}(t) \nsubseteq \mathcal{L}(s), s \prec t$, and $s$ is not blocked, then set $\mathcal{L}(s):=\mathcal{L}(s) \cup \mathcal{L}(t)$

Figure 11: The completion rules for $\mathcal{S H O} \mathcal{O K}(\mathcal{D})$. 
tight complexity bound for $\mathcal{S H O} \mathcal{Q K}(\mathcal{D})$ : the following corollary is an easy by-product of the correctness proofs (for a proof see again Appendix C).

Corollary 4.3. If a $\mathcal{S H O} \mathcal{Q} \mathcal{K}(\mathcal{D})$-concept $D$ is satisfiable w.r.t. a role box $\mathcal{R}$ and a path-free key box $\mathcal{K}$, then $D$ is satisfiable w.r.t. $\mathcal{R}$ and $\mathcal{K}$ in a model of size at most $\left|\Delta_{\mathcal{I}}\right| \leq 2^{m}$ for $m=\# \mathrm{cl}^{+}(D, \mathcal{R}, \mathcal{K})$.

Thus the following is an alternative algorithm for deciding satisfiability of a $\mathcal{S H O} \mathcal{Q K}(\mathcal{D})$ concept $D$ w.r.t. a role box $\mathcal{R}$ and a path-free key box $\mathcal{K}$ : first, guess an interpretation $\mathcal{I}$ with cardinality of $\Delta_{\mathcal{I}}$ bounded by $2^{m}$, using placeholder variables from $\mathrm{O}_{c}$ instead of concrete values in the interpretation of concrete features. Let $V_{c}$ be the set of variables from $\mathrm{O}_{\mathrm{c}}$ occuring in $\mathcal{I}$. Additionally guess an "interpretation" $\mathcal{P}$ for the concrete domain predicates: just as in completion forests, $\mathcal{P}$ maps each $n$-ary concrete predicate used in $D$ or $\mathcal{K}$ to an $n$-ary relation on $V_{c}$. Then perform standard (polynomial-time) model checking to ensure that $\mathcal{I}$ is a model of $D$. In doing this, treat concepts of the form $\exists g_{1}, \ldots, g_{n} . P$ using the interpretation of predicates $\mathcal{P}$. It is easily checked in polynomial time that $\mathcal{I}$ is also a model of $\mathcal{R}$ and $\mathcal{K}$ - for the latter, assume that all placeholder variables stand for different values. Finally, use the concrete domain $\mathcal{D}$-tester to check whether the conjunction

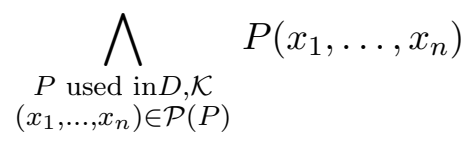

is satisfiable. Answer "yes" if it is and "no" otherwise. Since this algorithm can clearly be implemented in NEXPTIME provided that there is a $\mathcal{D}$-tester running in non-deterministic polynomial time, we obtain the following:

Theorem 4.4. Let $\mathcal{D}$ be a key-admissible concrete domain such that extended $\mathcal{D}$-satisfiability is in $\mathrm{NP}$, then $\mathcal{S H O} \mathcal{O K}(\mathcal{D})$-concept satisfiability w.r.t. TBoxes, role boxes, and path-free key boxes is in NEXPTIME.

\section{Conclusion}

In this paper, we have identified key constraints as an interesting extension of description logics with concrete domains. Starting from this observation, we introduced a number of natural description logics and provided a comprehensive analysis of the decidability and complexity of reasoning. The main observation of our investigations is that key boxes can have dramatic consequences on the complexity of reasoning: for example, the PSPACEcomplete DL $\mathcal{A L C}(\mathcal{D})$ becomes NExPTime-complete if extended with path-free, unary, Boolean key boxes and undecidable if extended with path-free, unary, non-Boolean key boxes. Thus the effect of our key boxes on the complexity are quite different from the effect of key assertions where only abstract features are allowed (Calvanese et al., 2000): these abstract key assertions can be said to be "for free" since they do not increase the complexity for expressive description logics.

We show that the restriction to Boolean key boxes (in the $\mathcal{A L C O K}(\mathcal{D})$ case) and to path-free key boxes (in the $\mathcal{S H O} \mathcal{O K}(\mathcal{D})$ case) yield decidabile and NExpTime-complete reasoning problems. We selected $\mathcal{A L C}(\mathcal{D})$ and $\mathcal{S H O} \mathcal{Q}(\mathcal{D})$ as the basis for our analysis since, 
in our opinion, these are the most fundamental description logics with concrete domains. Going one step further, it would be interesting to combine key boxes with other extensions of concrete domains, such as the ones presented by Lutz $(2003,2004)$. To name only one possibility, the extension of both $\mathcal{A L C O K}(\mathcal{D})$ and $\mathcal{S H O Q Q}(\mathcal{D})$ with inverse roles seems to be a natural idea. Note that inverse roles interact with several of the available means of expressivity: while $\mathcal{A L C}$ with inverse roles is PSPACE complete (Horrocks, Sattler, \& Tobies, 1999), $\mathcal{A} \mathcal{L C O}$ with inverse roles is ExpTime-complete (Areces et al., 1999) and $\mathcal{A L C}(\mathcal{D})$ with inverse roles even NExPTime-complete (Lutz, 2004).

Other options for future research are more closely related to the material presented in this paper. For example, is $\mathcal{S H O} \mathcal{Q K}(\mathcal{D})$-concept satisfiability still decidable if we drop the requirement of key boxes to be path-free? Moreover, we had to leave the exact time requirements of our tableau algorithm as an open problem. If this algorithm runs in (nondeterministic) exponential time, it directly yields Theorem 4.4 rather than via a bounded model property.

\section{Acknowledgments}

We would like to thank the anonymous reviewers for valuable comments. This paper is an extended version of (Lutz, Areces, Horrocks, \& Sattler, 2003).

\section{Appendix A. Proofs of Section 3.2}

We prove that $\mathrm{D}_{2}$-satisfiability can be decided in PTIME.

Proposition A.1. $\mathrm{D}_{2}$-satisfiability is in PTIME.

Proof. Let $c$ be a $D_{2}$-conjunction. We show that $c$ is satisfiable iff none of the following conditions applies:

1. $c$ contains a conjunct $\perp_{\mathrm{D}_{2}}(x)$;

2. $c$ contains conjuncts $\operatorname{bit}_{k}^{i}(x)$ and $\operatorname{bit}_{k}^{i}(x)$;

3. $c$ contains conjuncts bit $n_{k}^{i}(x)$ and bit $m_{\ell}^{j}(x)$ with $k \neq \ell$;

4. $c$ contains conjuncts $\operatorname{bit} n_{k}^{i}(x)$ and $\overline{\operatorname{bit} n_{k}^{i}}(x)$;

5. $c$ contains conjuncts bit $n_{k}^{i}(x), \overline{\operatorname{bit}_{k}^{j}}(x)$, and $\overline{\operatorname{bit} 1_{k}^{j}}(x)$.

It is easily seen that $c$ is unsatisfiable if one of the conditions applies. Assume now that Conditions 1 to 5 do not apply to $c$ and let $X$ be the set of variables used in $c$. For each $x \in X$, set $t(x)=k$ if $\operatorname{bit} n_{k}^{i}(x) \in c$ for some $n, i \in \mathbb{N}$. ${ }^{6}$ If $\operatorname{bit} n_{k}^{i}(x) \notin c$ for all $n, i, k \in \mathbb{N}$, then set $t(x)=r$ for some $r$ not appearing as an index $\cdot_{r}$ to a predicate in $c$. The mapping $t$ is well-defined since $c$ is finite, Condition 3 does not apply, and the only predicates available are $\operatorname{bit} n_{k}^{i}(\cdot), \perp_{\mathrm{D}_{2}}(\cdot)$, and $\top_{\mathrm{D}_{2}}(\cdot)$. We define a solution $\delta$ for $c$ as follows: for each $x \in X$, set $\delta(x)$ to the bit vector $v \in \operatorname{BV}_{t(x)}$ in which the $i^{\prime}$ th bit is 1 if bit $1_{t(x)}^{i}(x) \in c$ or $\overline{\operatorname{bit}_{t(x)}^{i}}(x) \in c$, and 0 otherwise. It remains to prove that $\delta$ is indeed a solution for $c$ :

6. We use " $P(x) \in c$ " as an abbreviation for " $P(x)$ is a conjunct in $c$ ". 
- Let $\operatorname{bit}_{k}^{i}(x) \in c$. Then $t(x)=k$ and thus $\delta(x) \in \mathrm{BV}_{k}$. Since Condition 2 does not apply, we have $\operatorname{bit}_{k}^{i}(x) \notin c$. Moreover, non-applicability of Condition 4 implies $\overline{\operatorname{bit}_{k}^{i}}(x) \notin c$. By definition of $\delta$, the $i$ 'th bit of $\delta(x)$ is thus 0 .

- Let $\operatorname{bit}_{k}^{i}(x) \in c$. Then $t(x)=k$ and $\delta(x) \in \mathrm{BV}_{k}$. By definition of $\delta$, the $i$ 'th bit of $\delta(x)$ is 1 .

- Let $\overline{\operatorname{bit}_{k}^{i}}(x) \in c$. If $t(x) \neq k$, then $\delta(x) \notin \mathrm{BV}_{k}$. Thus $\delta(x) \in\left(\overline{\operatorname{bit}_{k}^{i}}\right)^{\mathrm{D}_{2}}$ and we are done. If $t(x)=k$, then the $i$ 'th bit of $\delta(x)$ is 1 by definition of $\delta$ and thus again $\delta(x) \in\left(\overline{\operatorname{bit}_{k}^{i}}\right)^{\mathrm{D}_{2}}$.

- Let $\overline{\operatorname{bit} 1_{k}^{i}}(x) \in c$. If $t(x) \neq k$, then $\delta(x) \notin \mathrm{BV}_{k}$ and we are done. If $t(x)=k$, then bit $n_{k}^{j}(x) \in c$ for some $n, j \in \mathbb{N}$. Since Condition 5 does not apply, we thus have $\overline{\operatorname{bit}_{k}^{i}}(x) \notin c$. Moreover, non-applicability of Condition 4 yields bit $1_{k}^{i}(x) \notin c$. Thus, by definition of $\delta$, the $i$ 'th bit of $\delta(x)$ is 0 .

It is obvious that the listed properties can be checked in polynomial time.

\section{Appendix B. Proofs of Section 4.1}

We prove termination, soundness, and completeness of the $\mathcal{A L C O K}(\mathcal{D})$ tableau algorithm presented in Section 4.1, starting with termination. We start with establishing a few notions and technical lemmas.

Let $C$ be a concept and $\mathcal{K}$ a key box. We use $|C|$ to denote the length of $C$, i.e. the number of symbols used to write it down, and $|\mathcal{K}|$ to denote $\sum_{\left(u_{1}, \ldots, u_{k} \text { keyfor } C\right) \in \mathcal{K}}|C|$. For a path $u=f_{1} \cdots f_{k} g$, we use $|u|$ to denote $k+1$. The role depth of concepts is defined inductively as follows:

$$
\begin{aligned}
& \operatorname{rd}(A)=\operatorname{rd}(N)=\operatorname{rd}(g \uparrow)=0 \\
& \operatorname{rd}\left(\exists u_{1}, \ldots, u_{n} . P\right)=\max \left\{\left|u_{i}\right| \mid 1 \leq i \leq n\right\}-1 \\
& \operatorname{rd}(C \sqcap D)=\operatorname{rd}(C \sqcup D)=\max \{\operatorname{rd}(C), \operatorname{rd}(D)\} \\
& \operatorname{rd}(\exists R . C)=\operatorname{rd}(\forall R . C)=\operatorname{rd}(C)+1 .
\end{aligned}
$$

The following series of lemmas will eventually allow us to prove termination.

Lemma B.1. There is a constant $k$ such that, if the tableau algorithm is started on input $C_{0}, \mathcal{K}$ and $\mathbf{T}=\left(V_{a}, V_{c}, E, \mathcal{L}\right)$ is a completion tree constructed during the run of the algorithm, then $\# V_{a} \leq 2^{\left|C_{0}\right|^{k}}$ and $\# V_{c} \leq 2^{\left|C_{0}\right|^{k}}$.

Proof. Using induction on the number of rule applications and a case distinction according to the applied rule, it is straightforward to show that

$$
C \in \mathcal{L}(a) \text { implies } \operatorname{rd}(C) \leq\left|C_{0}\right|-\operatorname{lev}_{\mathbf{T}}(a)
$$

for all constructed completion trees $\mathbf{T}$. We omit the details but note that, (1) for treating the Rch rule, one needs to employ the fact that $\mathcal{K}$ is Boolean and thus only adds concepts of role depth 0 to node labels, and (2) for treating the Rp rule, we use that $a \prec b$ implies $\operatorname{lev}_{\mathbf{T}}(a) \leq \operatorname{lev}_{\mathbf{T}}(b)$. 
This implies an upper bound on the depth of constructed completion trees: first, only the $\mathrm{R} \exists$ and $\mathrm{R} \exists \mathrm{c}$ rules generate new nodes, and an application of either rule to a node $a \in V_{a}$ implies $\mathcal{L}(a) \neq \emptyset$ and thus $\operatorname{lev}_{\mathbf{T}}(a) \leq\left|C_{0}\right|$ by $(*)$. Second, each new (abstract or concrete) node $b$ generated by an application of these rules to a node $a \in V_{a}$ clearly satisfies $\operatorname{lev}_{\mathbf{T}}(b) \leq \operatorname{lev}_{\mathbf{T}}(a)+\max \left(1, \operatorname{mpl}\left(C_{0}\right)\right)$, where $\operatorname{mpl}\left(C_{0}\right)$ denotes the maximum length of paths in $C_{0}$ (note that concepts in $\mathcal{K}$ may not contain any paths since it is Boolean). Since $\mathrm{mpl}\left(C_{0}\right) \leq\left|C_{0}\right|$, the above observations imply that the depth of constructed completion trees is bounded by $2 \cdot\left|C_{0}\right|$.

Now for the out-degree. If a node $a$ is generated, then this is due to the application of a rule $\mathrm{R} \exists$ or $\mathrm{R} \exists \mathrm{c}$ and, initially, $a$ has at most one successor. Let us analyze the number of successors generated by later applications of the rules $\mathrm{R} \exists$ and $\mathrm{R} \exists$ c to $a$ : these rules can be applied at most once for each concept of the form $\exists R . C$ or $\exists u_{1}, \ldots, u_{n} . P$ in $\mathcal{L}(a)$. By definition of $\operatorname{cl}\left(C_{0}, \mathcal{K}\right)$ and since $\mathcal{K}$ is Boolean, the number of such concepts per node label is bounded by \# $\operatorname{sub}\left(C_{0}\right) \leq\left|C_{0}\right|$. Moreover, each rule application creates at most $\left|C_{0}\right|$ successors. Hence, the out-degree of constructed completion trees is bounded by $\left|C_{0}\right|^{2}+1$.

Lemma B.2. There is a constant $k$ such that, if the tableau algorithm is started with $C_{0}, \mathcal{K}$, then, in every recursion step, the while loop terminates after at most $2^{\left|C_{0}\right|^{k}}$ steps.

Proof. Fix an argument $S=(\mathbf{T}, \mathcal{P}, \prec, \sim)$ with $\mathbf{T}=\left(V_{a}, V_{c}, E, \mathcal{L}\right)$ passed to the sat function, let $\sim_{1}, \sim_{2}, \ldots$ be the sequence of concrete equivalences computed in the while loop, and let $\approx_{\mathrm{c}}^{1}, \approx_{\mathrm{c}}^{2}, \ldots$ be the corresponding $\approx_{\mathrm{c}}$ relations. Since test $\left(\zeta_{S}\right)$ calls a $\mathcal{D}$-tester, each of these calls indeed terminates.

We show that

$$
\sim_{1} \subsetneq \sim_{2} \subsetneq \cdots
$$

which implies Lemma B.2: by Lemma B.1, there exists a constant $k$ such that $\# V_{c} \leq 2^{\left|C_{0}\right|^{k}}$. Hence, we have $\# \sim_{i} \leq 2^{2 \cdot\left|C_{0}\right|^{k}}$ which, together with $(*)$, implies that the number of steps performed by the while loop is also bounded by $2^{2 \cdot\left|C_{0}\right|^{k}}$.

Now for the proof of $(*)$. If the while loop reaches the $i$-th step, then we had $\sim_{i-1} \neq \approx_{\mathrm{c}}^{i-1}$ after step $i-1$. Since $\sim_{i-1} \subseteq \approx_{\mathrm{c}}^{i-1}$ by definition, this implies $\sim_{i-1} \subsetneq \approx_{\mathrm{c}}^{i-1}$. By definition of $\zeta_{S}$, it is easy to see that $\approx_{\mathrm{c}}^{i-1} \subseteq \sim_{i}$ for $i \geq 1$. Hence $\sim_{i-1} \subsetneq \sim_{i}$.

Lemma B.3. There is a constant $k$ such that, if the tableau algorithm is started with $C_{0}, \mathcal{K}$, then the number of recursive calls is bounded by $2^{\left(\left|C_{0}\right|+|\mathcal{K}|\right)^{k}}$.

Proof. It obviously suffices to establish an appropriate upper bound on the number of rule applications. The $\mathrm{R} \sqcap, \mathrm{R} \sqcup, \mathrm{R} \exists$, and $\mathrm{R} \exists$ c rules can be applied at most once for each concept in a node label. By Lemma B.1, the number of nodes is at most exponential in $\left|C_{0}\right|+|\mathcal{K}|$. Since neither nodes nor concepts in node labels are ever deleted, the fact that node labels are subsets of $\mathrm{cl}\left(C_{0}, \mathcal{K}\right)$ thus implies that the number of applications of these rules is at most exponential in $\left|C_{0}\right|+|\mathcal{K}|$. The same holds for the rules $\mathrm{R} \forall$ and $\mathrm{Rp}$, which can be applied at most once for every concept $C \in \mathrm{cl}\left(C_{0}, \mathcal{K}\right)$ and every pair of (abstract) nodes. Finally, the number of Rch applications is at most exponential in $\left|C_{0}\right|+|\mathcal{K}|$ since this rule can be applied at most once for every abstract node and every key assertion in $\mathcal{K}$. 
Termination is now an obvious consequence of Lemmas B.2 and B.3.

Corollary B.4 (Termination). The tableau algorithm terminates on any input.

Let us now prove soundness of the algorithm.

Lemma B.5 (Soundness). If the tableau algorithm returns satisfiable, then the input concept $C_{0}$ is satisfiable w.r.t. the input key box $\mathcal{K}$.

Proof. If the tableau algorithm returns satisfiable, then there exists a complete and clashfree completion system $S=(\mathbf{T}, \mathcal{P}, \prec, \sim)$ for $C_{0}$. Let $\mathbf{T}=\left(V_{a}, V_{c}, E, \mathcal{L}\right)$. By definition of the tableau algorithm, there is a completion system $S^{\prime}=\left(\mathbf{T}, \mathcal{P}, \prec, \sim^{\prime}\right)$ such that a call to test $\left(\zeta_{S^{\prime}}\right)$ returned $\sim$. Moreover, we have $\sim=\approx_{\mathrm{c}}$ in $S$. Thus, there exists a solution $\delta$ for $\zeta_{S^{\prime}}$ such that

$$
\delta(x)=\delta(y) \text { iff } x \approx_{\mathrm{c}} y .
$$

Clearly, $\delta$ is also a solution for $\zeta_{S}$ : since the second component $\mathcal{P}$ is the same in $S$ and $S^{\prime}$, $\delta$ is a solution for the first part $\bigwedge_{P \text { used in } C\left(x_{1}, \ldots, x_{n}\right) \in \mathcal{P}(P)} P\left(x_{1}, \ldots, x_{n}\right)$ of $\zeta_{S}$. Moreover, for each conjunct $=(x, y)$ from the second part of $\zeta_{S}$, we have $x \approx_{c} y$ by definition of $\zeta_{S}$ and thus $\delta(x)=\delta(y)$ by $(\dagger)$.

We now use $S$ and $\delta$ to construct an interpretation $\mathcal{I}$ by setting

$$
\begin{aligned}
\Delta_{\mathcal{I}} & =\left\{a \in V_{a} \mid \text { there is no } b \in V_{a} \text { such that } a \approx_{\mathrm{a}} b \text { and } b \prec a\right\} \cup\{w\} \\
A^{\mathcal{I}} & =\left\{a \in \Delta_{\mathcal{I}} \mid A \in \mathcal{L}(a)\right\} \\
N^{\mathcal{I}} & = \begin{cases}\left\{a \in \Delta_{\mathcal{I}} \mid N \in \mathcal{L}(a)\right\} & \text { if there is an } a \in \Delta_{\mathcal{I}} \text { such that } N \in \mathcal{L}(a) \\
\{w\} & \text { otherwise }\end{cases} \\
R^{\mathcal{I}} & =\left\{(a, b) \in \Delta_{\mathcal{I}} \times \Delta_{\mathcal{I}} \mid \begin{array}{r}
\text { there are } a^{\prime}, b^{\prime} \in V_{a} \text { such that } a \approx_{\mathrm{a}} a^{\prime}, b \approx_{\mathrm{a}} b^{\prime}, \text { and } \\
\left.b^{\prime} \text { is an } R \text {-successor of } a^{\prime}\right\}
\end{array}\right. \\
g^{\mathcal{I}} & =\left\{(a, \delta(x)) \in \Delta_{\mathcal{I}} \times \Delta_{\mathcal{D}} \mid x \text { is a } g / \approx_{\mathrm{a}} \text {-neighbor of } a\right\}
\end{aligned}
$$

for all $A \in \mathrm{N}_{\mathrm{C}}, N \in \mathrm{N}_{\mathrm{O}}, R \in \mathrm{N}_{\mathrm{R}}$, and $g \in \mathrm{N}_{\mathrm{cF}}$. We first show that $\mathcal{I}$ is well-defined:

- $N^{\mathcal{I}}$ is a singleton for each $N \in \mathrm{N}_{\mathrm{O}}$. Assume that there exist $a, b \in \Delta_{\mathcal{I}}$ such that $a \neq b$ and $N \in \mathcal{L}(a) \cap \mathcal{L}(b)$. By definition of $\approx_{a}$ (Definition 4.3), $N \in \mathcal{L}(a) \cap \mathcal{L}(b)$ implies $a \approx_{\mathrm{a}} b$. This, together with $a, b \in \Delta_{\mathcal{I}}$, yields $a \prec b$ and $b \prec a$, contradicting $\prec$ being a linear ordering.

- $f^{\mathcal{I}}$ is functional for each $f \in \mathrm{N}_{\mathrm{aF}}$. Assume that there exist $a, b, c \in \Delta_{\mathcal{I}}$ such that $\{(a, b),(a, c)\} \subseteq f^{\mathcal{I}}$ and $b \neq c$. Then there exist $a_{1}, a_{2}, b^{\prime}, c^{\prime} \in V_{a}$ such that $a \approx_{a} a_{1} \approx_{a}$ $a_{2}, b \approx_{a} b^{\prime}, c \approx_{a} c^{\prime}, b^{\prime}$ is an $f$-successor of $a_{1}$, and $c^{\prime}$ is an $f$-successor of $a_{2}$. By definition of $\approx_{a}$, we thus have $b^{\prime} \approx_{a} c^{\prime}$ implying $b \approx_{a} c$. Since $b, c \in \Delta_{\mathcal{I}}$, this yields $b \prec c$ and $c \prec b$, a contradiction.

- $g^{\mathcal{I}}$ is functional for each $g \in \mathrm{N}_{\mathrm{cF}}$. Assume that there exist an $a \in \Delta_{\mathcal{I}}$ and $x, y \in \mathrm{V}_{c}$ such that $\{(a, \delta(x)),(a, \delta(y))\} \subseteq f^{\mathcal{I}}$ and $\delta(x) \neq \delta(y)$. Then $x$ and $y$ are both $g / \approx_{a^{-}}$ neighbors of $a$. By definition of $\approx_{\mathrm{c}}$, we thus have $x \approx_{\mathrm{c}} y$ implying $\delta(x)=\delta(y)$ by $(\dagger)$, a contradiction. 
Now we show the following claim. In the proof, we use the notion of $f_{1} \cdots f_{k} / \approx_{a}$-neighbors (with $f_{1}, \ldots f_{k}$ abstract features), which is defined analogously to $u / \approx_{a}$-neighbors for paths $u$.

Claim 1: For all $a \in \Delta_{\mathcal{I}}$ and all paths $u$, we have $u^{\mathcal{I}}(a)=\alpha$ iff there is a $u_{i} / \approx_{a}$-neighbor $x$ of $a$ with $\delta(x)=\alpha$.

Proof: Let $u=f_{1} \cdots f_{k} g$. Using induction on $i$ it is easily proved that, for all $i$ and all $b \in \Delta_{\mathcal{I}}$, we have $f_{i}^{\mathcal{I}}\left(\cdots\left(f_{1}^{\mathcal{I}}(a)\right) \cdots\right)=b$ iff there is a $f_{1} \cdots f_{i} / \approx_{a}$-neighbor $b^{\prime}$ of $a$ with $b \approx_{a} b^{\prime}$. Thus we have in particular that $f_{k}^{\mathcal{I}}\left(\cdots\left(f_{1}^{\mathcal{I}}(a)\right) \cdots\right)=b$ iff there is an $f_{1} \cdots f_{k} / \approx_{a^{-}}$ neighbor $b^{\prime}$ of $a$ with $b \approx_{a} b^{\prime}$. To prove the claim, it hence remains to use the definition of $g^{\mathcal{I}}$ together with $(\dagger)$.

The following claim is central for showing that $\mathcal{I}$ is a model for $C_{0}$ and $\mathcal{K}$.

Claim 2: For all $a \in \Delta_{\mathcal{I}}$ and $C \in \operatorname{cl}\left(C_{0}, \mathcal{K}\right)$, if $C \in \mathcal{L}(a)$, then $a \in C^{\mathcal{I}}$.

Since $C_{0}$ is in the label of the root node, Claim 2 clearly implies that $\mathcal{I}$ is a model for $C_{0}$. Moreover, we can use it to prove that $\mathcal{I}$ satisfies all key assertions $\left(u_{1}, \ldots, u_{n}\right.$ keyfor $\left.C\right)$ in $\mathcal{K}$ : fix $a, b \in C^{\mathcal{I}}$ such that $u_{i}^{\mathcal{I}}(a)=u_{i}^{\mathcal{I}}(b)$ for $1 \leq i \leq n$. Non-applicability of Rch yields $\{C, \dot{\neg} C\} \cap \mathcal{L}(a) \neq \emptyset$. If $\dot{\rightarrow} C \in \mathcal{L}(a)$, then Claim 2 implies $a \in(\dot{\neg} C)^{\mathcal{I}}$ in contradiction to $a \in C^{\mathcal{I}}$. Thus we obtain $C \in \mathcal{L}(a)$. In an analogous way, we can argue that $C \in \mathcal{L}(b)$. Since $u_{i}^{\mathcal{I}}(a)$ and $u_{i}^{\mathcal{I}}(b)$ are defined for $1 \leq i \leq n$, Claim 1 yields that $a$ has a $u_{i} / \approx_{\text {a-neighbor }} x_{i}$ with $\delta\left(x_{i}\right)=u_{i}^{\mathcal{I}}(a)$ and $b$ a $u_{i} / \approx_{a}$-neighbor $y_{i}$ with $\delta\left(y_{i}\right)=u_{i}^{\mathcal{I}}(b)$ for $1 \leq i \leq n$. Thus the fact that $u_{i}^{\mathcal{I}}(a)=u_{i}^{\mathcal{I}}(b)$ yields $\delta\left(x_{i}\right)=\delta\left(y_{i}\right)$ for $1 \leq i \leq n$. By $(\dagger)$ we obtain $x_{i} \approx_{\mathrm{c}} y_{i}$ and thus $x_{i} \sim y_{i}$ for $1 \leq i \leq n$. By definition of $\approx_{a}$, we thus get $a \approx_{a} b$. Since $a, b \in \Delta_{\mathcal{I}}$, we obtain $a \nprec b$ and $b \nprec a$ by definition of $\Delta_{\mathcal{I}}$ and thus $a=b$.

It remains to prove Claim 2, which we do using structural induction:

- $C$ is a concept name or a nominal. Easy by construction of $\mathcal{I}$.

- $C=\neg D$. Since $C \in \operatorname{cl}\left(C_{0}, \mathcal{K}\right), C$ is in NNF and $D$ is a concept name. Since $S$ is clash-free, $C \in \mathcal{L}(a)$ implies $D \notin \mathcal{L}(a)$. Thus, $a \notin D^{\mathcal{I}}$ by construction of $\mathcal{I}$, which yields $a \in(\neg D)^{\mathcal{I}}$.

- $C=\exists u_{1}, \ldots, u_{n} . P$. Since the $\mathrm{R} \exists \mathrm{c}$ rule is not applicable, there exist $x_{1}, \ldots, x_{n} \in V_{c}$ such that $x_{i}$ is a $u_{i} / \approx_{a}$-neighbor of $a$ for $1 \leq i \leq n$ and $\left(x_{1}, \ldots, x_{n}\right) \in \mathcal{P}(P)$. Claim 1 yields $u_{i}^{\mathcal{I}}(a)=\delta\left(x_{i}\right)$ for $1 \leq i \leq n$. Since $\left(x_{1}, \ldots, x_{n}\right) \in \mathcal{P}(P)$ and $\delta$ is a solution for $\zeta_{S}$, we have $\left(\delta\left(x_{1}\right), \ldots, \delta\left(x_{n}\right)\right) \in P^{\mathcal{D}}$ and thus $a \in C^{\mathcal{I}}$.

- $C=g \uparrow$. Since $S$ is clash-free, there exists no $x \in V_{c}$ such that $x$ is $g / \approx_{a}$-neighbor of $a$. Thus by Claim 1 there is no $\alpha$ such that $(a, \alpha) \in g^{\mathcal{I}}$.

- $C=D \sqcap E$ or $C=D \sqcup E$. Straightforward using completeness and the induction hypothesis.

- $C=\exists R$.D. Since the $\mathrm{R} \exists$ rule is not applicable, $a$ has an $R / \approx_{\mathrm{a}}$-neighbor $b$ such that $D \in \mathcal{L}(b)$. Let $b^{\prime}$ be minimal w.r.t. $\prec$ such that $b \approx_{a} b^{\prime}$. By definition of $\mathcal{I}$, we have $\left(a, b^{\prime}\right) \in R^{\mathcal{I}}$. Non-applicability of the Rp rule yields $D \in \mathcal{L}\left(b^{\prime}\right)$. By induction, we get $b^{\prime} \in D^{\mathcal{I}}$ and thus $a \in C^{\mathcal{I}}$. 
- $C=\forall R$.D. Let $(a, b) \in R^{\mathcal{I}}$. By definition of $\mathcal{I}$, this implies that there exist $a^{\prime}, b^{\prime} \in V_{a}$ such that $a$ is minimal w.r.t. $\prec$ and $a \approx_{a} a^{\prime}, b$ is minimal w.r.t. $\prec$ and $b \approx_{a} b^{\prime}$, and $b^{\prime}$ is an $R$-successor of $a^{\prime}$. Since $b^{\prime}$ is clearly an $R / \approx_{a}$-neighbor of $a$, non-applicability of $\mathrm{R} \forall$ yields $D \in \mathcal{L}\left(b^{\prime}\right)$, which implies $D \in \mathcal{L}(b)$ due to the non-applicability of Rp. By induction, we get $b \in D^{\mathcal{I}}$. Since this holds independently of the choice of $b$, we obtain $a \in(\forall R . D)^{\mathcal{I}}$.

Lemma B.6 (Completeness). If the input concept $C_{0}$ is satisfiable w.r.t. the input key box $\mathcal{K}$, then the tableau algorithm returns satisfiable.

Proof. Let $\mathcal{I}$ be a model of $C_{0}$ and $\mathcal{K}$. We use $\mathcal{I}$ to "guide" (the non-deterministic parts of) the algorithm such that it constructs a complete and clash-free completion system. A completion system $S=(\mathbf{T}, \mathcal{P}, \prec, \sim)$ with $\mathbf{T}=\left(V_{a}, V_{c}, E, \mathcal{L}\right)$ is called $\mathcal{I}$-compatible if there exist mappings $\pi: V_{a} \rightarrow \Delta_{\mathcal{I}}$ and $\tau: V_{c} \rightarrow \Delta_{\mathcal{D}}$ such that

(Ca) $C \in \mathcal{L}(a) \Rightarrow \pi(a) \in C^{\mathcal{I}}$

(Cb) $b$ is an $R$-successor of $a \Rightarrow(\pi(a), \pi(b)) \in R^{\mathcal{I}}$

(Cc) $x$ is a $g$-successor of $a \Rightarrow g^{\mathcal{I}}(\pi(a))=\tau(x)$

(Cd) $\left(x_{1}, \ldots, x_{n}\right) \in \mathcal{P}(P) \Rightarrow\left(\tau\left(x_{1}\right), \ldots, \tau\left(x_{n}\right)\right) \in P^{\mathcal{D}}$

(Ce) $x \sim y \Rightarrow \tau(x)=\tau(y)$.

We first establish the following claim:

Claim 1: If a completion system $S$ is $\mathcal{I}$-compatible, then (i) $a \approx_{a} b$ implies $\pi(a)=\pi(b)$ and (ii) $x \approx_{\mathrm{c}} y$ implies $\tau(x)=\tau(y)$.

Proof: We show by induction on $i$ that $a \approx_{a}^{i} b$ implies $\pi(a)=\pi(b)$ (see Definition 4.3), which yields (i).

- Start. If $a \approx_{a}^{0} b$, then there exists a nominal $N$ such that $N \in \mathcal{L}(a) \cap \mathcal{L}(b)$. By (Ca) we obtain $\pi(a) \in N^{\mathcal{I}}$ and $\pi(b) \in N^{\mathcal{I}}$, which yields $\pi(a)=\pi(b)$ by definition of the semantics.

- Step. For $a \approx_{\mathrm{a}}^{i} b$, we distinguish three cases:

1. If $a \approx_{\mathrm{a}}^{i-1} b$, then $\pi(a)=\pi(b)$ by induction.

2. There is a $c \in V_{a}$ and an $f \in \mathrm{N}_{\mathrm{aF}}$ such that both $a$ and $b$ are $f / \approx_{\mathrm{a}}^{i-1}$-neighbors of $c$. Hence, there exist $c_{1}, c_{2} \in V_{a}$ such that $c \approx_{\mathrm{a}}^{i-1} c_{1} \approx_{\mathrm{a}}^{i-1} c_{2}, a$ is an $f$-successor of $c_{1}$, and $b$ is an $f$-successor of $c_{2}$. By induction, we have $\pi(c)=\pi\left(c_{1}\right)=\pi\left(c_{2}\right)$. Thus (Cb) yields $\{(\pi(c), \pi(a)),(\pi(c), \pi(b))\} \subseteq f^{\mathcal{I}}$, which implies $\pi(a)=\pi(b)$ by definition of the semantics.

3. There exist $\left(u_{1}, \ldots, u_{n}\right.$ keyfor $\left.C\right) \in \mathcal{K}, u_{i} / \approx_{\mathrm{a}}^{i-1}$-neighbors $x_{i}$ of $a$ and $u_{i} / \approx_{\mathrm{a}}^{i-1}$ neighbors $y_{i}$ of $b$ for $1 \leq i \leq n$ such that $C \in \mathcal{L}(a) \cap \mathcal{L}(b)$ and $x_{i} \sim y_{i}$ for $1 \leq i \leq n$. (Ca) yields $a, b \in C^{\mathcal{I}}$. Using induction, $(\mathrm{Cb})$, and (Cc), it is straightforward to 
show that $u_{i}^{\mathcal{I}}(\pi(a))=\tau\left(x_{i}\right)$ and $u_{i}^{\mathcal{I}}(\pi(b))=\tau\left(y_{i}\right)$ for $1 \leq i \leq n$. By (Ce), this implies $u_{i}^{\mathcal{I}}(\pi(a))=u_{i}^{\mathcal{I}}(\pi(b))$ for $1 \leq i \leq k$. Since $\mathcal{I}$ is a model of the key box $\mathcal{K}$, this yields $\pi(a)=\pi(b)$ by definition of the semantics.

Now for Part (ii) of Claim 1. If $x \approx_{\mathrm{c}} y$, then either $x \sim y$ or there is an $a \in V_{a}$ and a $g \in \mathrm{N}_{\mathrm{cF}}$ such that both $x$ and $y$ are $g / \approx_{a}$-neighbors of $a$. In the former case, (Ce) yields $\tau(x)=\tau(y)$. In the latter case, Part (i) of the claim and (Cc) yields $\{(\pi(a), \tau(x)),(\pi(a), \tau(y))\} \subseteq g^{\mathcal{I}}$ which implies $\tau(x)=\tau(y)$. This finishes the proof of Claim 1 .

We can now show that the completion rules can be applied such that $\mathcal{I}$-compatibility is preserved.

Claim 2: If a completion system $S$ is $\mathcal{I}$-compatible and a rule $\mathrm{R}$ is applicable to $S$, then $\mathrm{R}$ can be applied such that an $\mathcal{I}$-compatible completion system $S^{\prime}$ is obtained.

Proof: Let $S$ be an $\mathcal{I}$-compatible completion system, let $\pi$ and $\tau$ be functions satisfying (Ca) to (Ce), and let $\mathrm{R}$ be a completion rule applicable to $S$. We make a case distinction according to the type of $\mathrm{R}$.

$\mathrm{R} \sqcap$ The rule is applied to a concept $C_{1} \sqcap C_{2} \in \mathcal{L}(a)$. By (Ca), $C_{1} \sqcap C_{2} \in \mathcal{L}(a)$ implies $\pi(a) \in\left(C_{1} \sqcap C_{2}\right)^{\mathcal{I}}$ and hence $\pi(a) \in C_{1}^{\mathcal{I}}$ and $\pi(a) \in C_{2}^{\mathcal{I}}$. Since the rule adds $C_{1}$ and $C_{2}$ to $\mathcal{L}(a)$, it yields a completion system that is $\mathcal{I}$-compatible via the same $\pi$ and $\tau$.

$\mathrm{R} \sqcup$ The rule is applied to $C_{1} \sqcup C_{2} \in \mathcal{L}(a) . C_{1} \sqcup C_{2} \in \mathcal{L}(a)$ implies $\pi(a) \in C_{1}^{\mathcal{I}}$ or $\pi(a) \in C_{2}^{\mathcal{I}}$. Since the rule adds either $C_{1}$ or $C_{2}$ to $\mathcal{L}(a)$, it can be applied such that it yields a completion system that is $\mathcal{I}$-compatible via the same $\pi$ and $\tau$.

$\mathrm{R} \exists$ The rule is applied to a concept $\exists R . C \in \mathcal{L}(a)$, generates a new $R$-successor $b$ of $a$ and sets $\mathcal{L}(b)=\{C\}$. By $(\mathrm{Ca})$, we have $\pi(a) \in(\exists R . C)^{\mathcal{I}}$ and, hence, there exists a $d \in \Delta_{\mathcal{I}}$ such that $(\pi(a), d) \in R^{\mathcal{I}}$ and $d \in C^{\mathcal{I}}$. Set $\pi^{\prime}:=\pi \cup\{b \mapsto d\}$. It is readily checked that the resulting completion system is $\mathcal{I}$-compatible via $\pi^{\prime}$ and $\tau$.

$\mathrm{R} \forall$ The rule is applied to a concept $\forall R . C \in \mathcal{L}(a)$ and adds $C$ to $\mathcal{L}(b)$ of an existing $R / \approx_{a}$-neighbor $b$ of $a$. Hence, there exists an $a^{\prime}$ such that $a \approx_{a} a^{\prime}$ and $b$ is an $R$ successor of $a^{\prime}$. By Part (i) of Claim 1, we have $\pi(a)=\pi\left(a^{\prime}\right)$. Thus, by (Ca) we have $\pi\left(a^{\prime}\right) \in(\forall R . C)^{\mathcal{I}}$ while $(\mathrm{Cb})$ yields $\left(\left(\pi\left(a^{\prime}\right), \pi(b)\right) \in R^{\mathcal{I}}\right.$. By definition of the semantics, $\pi(b) \in C^{\mathcal{I}}$ and thus the resulting completion system is $\mathcal{I}$-compatible via $\pi$ and $\tau$.

$\mathrm{R} \exists \mathrm{c}$ The rule is applied to a concept $\exists u_{1}, \ldots, u_{n} . P \in \mathcal{L}(a)$ with $u_{i}=f_{1}^{(i)} \cdots f_{k_{i}}^{(i)} g_{i}$ for $1 \leq i \leq n$. The rule application generates new abstract nodes $b_{j}^{(i)}$ and $x_{j}$ for $1 \leq i \leq n$ and $1 \leq j \leq k_{i}$ such that

$-b_{1}^{(i)}$ is an $f_{1}^{(i)}$-successor of $a$ for $1 \leq i \leq n$,

$-b_{j}^{(i)}$ is an $f_{j}^{(i)}$-successor of $b_{j-1}^{(i)}$ for $1 \leq i \leq n$ and $2 \leq j \leq k_{i}$,

- $x_{i}$ is $g_{i}$-successor of $b_{k_{i}}^{(i)}$ for $1 \leq i \leq n$, and

- $\left(x_{1}, \ldots, x_{n}\right) \in \mathcal{P}(P)$. 
By (Ca), we have $\pi(a) \in\left(\exists u_{1}, \ldots, u_{n} . P\right)^{\mathcal{I}}$. Hence, there exist $d_{j}^{(i)} \in \Delta_{\mathcal{I}}$ for $1 \leq i \leq n$ and $1 \leq j \leq k_{i}$ and $\alpha_{1}, \ldots, \alpha_{n} \in \Delta_{\mathcal{D}}$ such that

$-\left(\pi(a), d_{1}^{(i)}\right) \in\left(f_{1}^{(i)}\right)^{\mathcal{I}}$ for $1 \leq i \leq n$,

$-\left(d_{j-1}^{(i)}, d_{j}^{(i)}\right) \in\left(f_{j}^{(i)}\right)^{\mathcal{I}}$ for $1 \leq i \leq n$ and $2 \leq j \leq k_{i}$,

$-g_{i}^{\mathcal{I}}\left(d_{k_{i}}^{(i)}\right)=\alpha_{i}$ for $1 \leq i \leq n$, and

$-\left(\alpha_{1}, \ldots, \alpha_{n}\right) \in P^{\mathcal{D}}$.

Set $\pi^{\prime}:=\pi \cup \bigcup_{1 \leq i \leq n}$ and $1 \leq j \leq k_{i}\left\{b_{j}^{(i)} \mapsto d_{j}^{(i)}\right\}$ and $\tau^{\prime}:=\tau \cup \bigcup_{1 \leq i \leq n}\left\{x_{i} \mapsto \alpha_{i}\right\}$.

The resulting completion system is $\mathcal{I}$-compatible via $\pi^{\prime}$ and $\tau^{\prime}$.

Rch The rule is applied to an abstract node $a$ and a key assertion $\left(u_{1}, \ldots, u_{n}\right.$ keyfor $\left.C\right)$ $\in \mathcal{K}$ and non-deterministically adds either $C$ or $\rightarrow C$. By definition of the semantics, $\pi(a) \in C^{\mathcal{I}}$ or $\pi(a) \in(\dot{\neg} C)^{\mathcal{I}}$. Hence, Rch can be applied such that the resulting completion system is $\mathcal{I}$-compatible via $\pi$ and $\tau$.

Rp The rule is applied to a concept $C \in \mathcal{L}(a)$ and adds $C$ to the label $\mathcal{L}(b)$ of a node $b$ with $a \approx_{\mathrm{a}} b$. By $(\mathrm{Ca})$, we have $\pi(a) \in C^{\mathcal{I}}$. Since Claim 1 yields $\pi(a)=\pi(b)$, it follows that the resulting completion system is $\mathcal{I}$-compatible via $\pi$ and $\tau$.

Finally, we show that $\mathcal{I}$-compatibility implies clash-freeness.

Claim 3: Every $\mathcal{I}$-compatible completion system is clash-free.

Proof: Let $S=(\mathbf{T}, \mathcal{P}, \prec, \sim)$ be an $\mathcal{I}$-compatible completion system. Consider the three kinds of a clash:

- Due to (Ca), a clash of the form $\{A, \neg A\} \in \mathcal{L}(a)$ clearly contradicts the semantics.

- Assume that there are $a \in V_{a}$ and $x \in V_{c}$ such that $g \uparrow \in \mathcal{L}(a)$ and $x$ is $g / \approx_{\mathrm{a}}$-neighbor of $a$. Then there exists $b \in V_{a}$ such that $a \approx_{a} b$ and $x$ is $g$-successor of $b$. By Claim 1 , $a \approx_{\mathrm{a}} b$ implies $\pi(a)=\pi(b)$. Thus, $g \uparrow \in \mathcal{L}(a)$ and $(\mathrm{Ca})$ give $\pi(b) \in(g \uparrow)^{\mathcal{I}}$. We obtain a contradiction since $(\mathrm{Cc})$ yields $(\pi(b), \tau(x)) \in g^{\mathcal{I}}$.

- Properties (Cd) and (Ce) and Part (ii) of Claim 1 imply that $\tau$ is a solution for $\zeta_{S}$. Thus, $S$ is concrete domain satisfiable.

We can now describe the "guidance" of the tableau algorithm by the model $\mathcal{I}$ in detail: we ensure that, at all times, the considered completion systems are $\mathcal{I}$-compatible. This obviously holds for the initial completion system

$$
S_{C_{0}}=\left(\mathbf{T}_{C_{0}}, \mathcal{P}_{\emptyset}, \emptyset, \emptyset\right) \text { with } \mathbf{T}_{C_{0}}=\left(\left\{a_{0}\right\}, \emptyset, \emptyset,\left\{a_{0} \mapsto\{C\}\right\}\right)
$$

We guide the non-deterministic test function such that, when given a predicate conjunction $\zeta_{S}$ with set of variables $V_{c} \subseteq \mathrm{O}_{\mathrm{c}}$ as input, it returns the relation $\sim$ defined by setting $x \sim y$ iff $\tau(x)=\tau(y)$ for all $x, y \in V$. The relation $\sim$ is a concrete equivalence since $\tau$ is a solution for $\zeta_{S}$ (see above). With this guidance, $(\mathrm{Ce})$ is obviously satisfied after each call to test, and the other properties are not affected by such a call. According to Claim 2, we can apply the 
completion rules such that $\mathcal{I}$-compatibility is preserved. By Corollary B.4, the algorithm always terminates, hence also when guided in this way. Since, by Claim 3, we will not find a clash, the algorithm returns satisfiable.

The tableau algorithm yields decidability and a tight upper complexity bound for $\mathcal{A L C O K}(\mathcal{D})$ concept satisfiability w.r.t. key boxes.

Theorem B.7 (Theorem 4.1 of Section 4.1). Let $\mathcal{D}$ be a key-admissible concrete domain. If extended $\mathcal{D}$-satisfiability is in $\mathrm{NP}$, then $\mathcal{A} \mathcal{L C O} \mathcal{K}(\mathcal{D})$-concept satisfiability w.r.t. Boolean key boxes is in NExPTIME.

Proof. Corollary B.4 and Lemmas B.5 and B.6 yield decidability of $\mathcal{A} \mathcal{L C O} \mathcal{K}(\mathcal{D})$-concept satisfiability w.r.t. Boolean key boxes. For complexity, Lemma B.3 provides an exponential bound on the number of recursive calls. Hence, it remains to show that each single recursion step needs at most exponential time. By Lemma B.2, the while loop terminates after at most exponentially many steps. In each such step, we compute the relations $\approx_{a}$ and $\approx_{c}$, which are used in the construction of the predicate conjunction $\zeta_{S}$ and for checking termination of the while loop. Since, by Lemma B.1, there exists an exponential bound on the number of abstract and concrete nodes in the completion system $S$, this can obviously be done in exponential time. Moreover, Lemma B.1 implies that the size of $\zeta_{S}$ is at most exponential. This together with the fact that extended $\mathcal{D}$-satisfiability is in NP implies that the call to test needs at most exponential time. All remaining tasks (checking for clashes, completeness, and rule applicability) can clearly also be performed in exponential time.

\section{Appendix C. Proofs of Section 4.2}

We first provide the proof of Lemma 4.2 which shows that the notion of tableaux introduced in Section 4.2 is an adequate abstraction of models.

Lemma C.1 (Lemma 4.2 in Section 4.2). Let $D$ be a $\mathcal{S H O} \mathcal{Q} \mathcal{K}(\mathcal{D})$-concept in $N N F$, $\mathcal{R}$ a role box, and $\mathcal{K}$ a path-free key box in $N N F$. Then $D$ is satisfiable w.r.t. $\mathcal{R}$ and $\mathcal{K}$ iff $D$ has a tableau w.r.t. $\mathcal{R}$ and $\mathcal{K}$.

Proof. For the "only-if" direction, we construct a tableau $T$ from a common model $\mathcal{I}$ of $D$, $\mathcal{R}$, and $\mathcal{K}$ as follows:

$$
\begin{aligned}
\mathbf{S}_{a} & :=\Delta_{\mathcal{I}} \\
\mathbf{S}_{c} & :=\left\{x \in \Delta_{\mathcal{D}} \mid g^{\mathcal{I}}(s)=x \text { for some } s \in \mathbf{S}_{a}\right\} \\
\mathcal{L}(s) & :=\left\{C \in \operatorname{cl}(D, \mathcal{R}, \mathcal{K}) \mid s \in C^{\mathcal{I}}\right\} \\
E(s, t) & :=\left\{S \in \mathrm{N}_{\mathrm{R}}^{D, \mathcal{R}, \mathcal{K}} \mid(s, t) \in S^{\mathcal{I}}\right\} \\
e(s, g) & :=g^{\mathcal{I}}(s) \text { if } g^{\mathcal{I}}(s) \text { is defined } \\
\mathcal{P}(P) & :=\left\{\left(x_{1}, \ldots, x_{n}\right) \in \mathbf{S}_{c}^{n} \mid\left(x_{1}, \ldots, x_{n}\right) \in P^{\mathcal{D}}\right\} .
\end{aligned}
$$

It can be easily verified that $T$ is a tableau for $D$ w.r.t. $\mathcal{R}$ and $\mathcal{K}$ : the proof that $T$ satisfies (T1) - (T9) is identical to the corresponding cases in (Horrocks et al., 2000; Horrocks \& Sattler, 2001); (T10) holds by definition of $\mathcal{L}$; (T11) by definition of $\mathcal{L}$ and the fact that nominals are interpreted as singleton sets; (T12) by definition of $\mathcal{L}, e$, and $\mathcal{P}$ together with 
the semantics of concepts $\exists g_{1}, \ldots, g_{n} . P$; ( $\mathbf{T 1 3}$ ) since the identity function on $\mathbf{S}_{c}$ is clearly a solution for the listed predicate conjunction; (T14) by definition of $\mathcal{L}$ and $e$ together with the semantics of key constraints; and finally (T15) by definition of $\mathcal{L}$ and $e$ together with the semantics of concepts $g \uparrow$.

For the "if" direction, let $T=\left(\mathbf{S}_{a}, \mathbf{S}_{c}, \mathcal{L}, E, e, \mathcal{P}\right)$ be a tableau for $D$ w.r.t. $\mathcal{R}$ and $\mathcal{K}$ and let $\delta$ be a solution for the predicate conjunction in (T13). We construct a model $\mathcal{I}$ for $D$ as follows:

$$
\begin{aligned}
& \Delta_{\mathcal{I}}:=\mathbf{S}_{a} \\
& A^{\mathcal{I}}:=\left\{s \in \Delta_{\mathcal{I}} \mid A \in \mathcal{L}(s)\right\} \quad \text { for concept names } A \\
& N^{\mathcal{I}}:=\left\{s \in \Delta_{\mathcal{I}} \mid N \in \mathcal{L}(s)\right\} \text { for nominals } N
\end{aligned}
$$

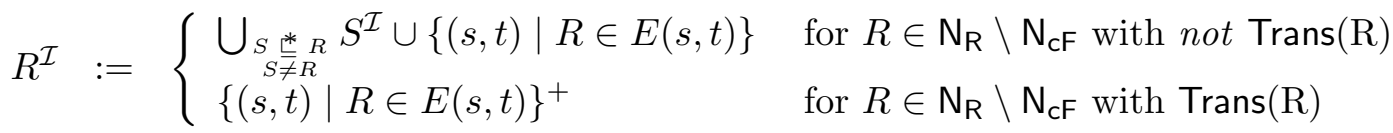

$$
\begin{aligned}
& g^{\mathcal{I}}(s):=\left\{\begin{array}{ll}
\delta(x) & \text { if } e(s, g)=x \\
\text { undefined } & \text { if } e(s, g) \text { is undefined }
\end{array} \text { for } g \in \mathrm{N}_{\mathrm{cF}} .\right.
\end{aligned}
$$

Due to (T11), the interpretation of nominals is a singleton. Moreover, the interpretation of roles is well-defined since role boxes are acyclic. The following claim is central for proving that $\mathcal{I}$ is indeed a model for $D, \mathcal{R}$, and $\mathcal{K}$ :

Claim: For each $C \in \operatorname{cl}(D, \mathcal{R}, \mathcal{K}), C \in \mathcal{L}(s)$ implies $s \in C^{\mathcal{I}}$.

Proof: We proceed by induction on the norm $\|C\|$ of $C$, which is defined as follows:

$$
\begin{array}{rll}
\|A\| & :=\|\neg A\| & :=0 \text { for } A \text { concept name } \\
\|g \uparrow\| & :=\left\|\exists u_{1}, \ldots, u_{n} . P\right\| & :=0 \\
\left\|C_{1} \sqcap C_{2}\right\| & :=\left\|C_{1} \sqcup C_{2}\right\| & :=1+\left\|C_{1}\right\|+\left\|C_{2}\right\| \\
\|(\geqslant n R C)\| & :=\|(\leqslant n R C)\| & :=1+\|C\|
\end{array}
$$

For concept names $A$ and nominals $N$, the claim follows by definition of $A^{\mathcal{I}}$ and $N^{\mathcal{I}}$. For the negation of concept names $A$ and nominals $N$ (note that $C$ is in NNF), the claim follows by definition of $A^{\mathcal{I}}$ and $N^{\mathcal{I}}$ together with (T1). Concepts $C$ of the form $C_{1} \sqcap C_{2}$ and $C_{1} \sqcup C_{2}$ can be treated using (T2) and (T3) together with the induction hypothesis. For existential, universal, and number restrictions, the proof is analogous to the one for $\mathcal{S H \mathcal { Q }}$ in (Horrocks et al., 2000). For concepts of the form $C=\exists g_{1}, \ldots g_{n} . P \in \mathcal{L}(s), s \in C^{\mathcal{I}}$ is an immediate consequence of (T12), the definition of $g_{i}^{\mathcal{I}}$, and the fact that $\left(x_{1}, \ldots, x_{n}\right) \in \mathcal{P}(P)$ implies $\left(\delta\left(x_{1}\right), \ldots, \delta\left(x_{n}\right)\right) \in P^{\mathcal{D}}$ by (T13). Finally, for concepts $C=g \uparrow, s \in C^{\mathcal{I}}$ is an immediate consequence of the definition of $g^{\mathcal{I}}$ together with (T15). This finishes the proof of the claim.

By definition of tableaux, there exists an $s_{0} \in \mathbf{S}_{a}$ such that $C \in \mathcal{L}\left(s_{0}\right)$. By the claim, $s_{0} \in C^{\mathcal{I}}$ and thus $\mathcal{I}$ is a model of $C$.

Next, we show that $\mathcal{I}$ is a model of $\mathcal{R}$. By definition of $R^{\mathcal{I}}$, it is obvious that $\operatorname{Trans}(R) \in$ $\mathcal{R}$ implies that $R^{\mathcal{I}}$ is a transitive relation. Now let $S \sqsubseteq R \in \mathcal{R}$. If $\operatorname{Trans}(R) \notin \mathcal{R}$, then we have $S^{\mathcal{I}} \subseteq R^{\mathcal{I}}$ by definition of $R^{\mathcal{I}}$. Now let $\operatorname{Trans}(R) \in \mathcal{R}$ and $(s, t) \in S^{\mathcal{I}}$. If $S \in E(s, t)$, then (T4) implies $R \in E(s, t)$, and thus $(s, t) \in R^{\mathcal{I}}$. Otherwise, there is an $S^{\prime} \stackrel{\Xi}{=}$ with $\operatorname{Trans}\left(S^{\prime}\right) \in \mathcal{R}$ and $(s, t) \in\left\{(u, v) \mid S^{\prime} \in E(u, v)\right\}^{+}$. Now (T4) together with $S^{\prime} \underline{\underline{E}} R$ implies 
that $\left\{(u, v) \mid S^{\prime} \in E(u, v)\right\} \subseteq\{(u, v) \mid R \in E(u, v)\}$, and thus $\operatorname{Trans}(R) \in \mathcal{R}$ implies that $(s, t) \in R^{\mathcal{I}}$.

It remains to show that $\mathcal{I}$ is a model of $\mathcal{K}$. To this end, let $\left(g_{1}, \ldots, g_{n}\right.$ keyfor $\left.C\right) \in \mathcal{K}$ and $s, t \in C^{\mathcal{I}}$ such that $g_{i}^{\mathcal{I}}(s)=g_{i}^{\mathcal{I}}(t)$ for $1 \leq i \leq n$. Since the predicate conjunction in (T13) contains explicit inequalities for all distinct concrete individuals, this implies that $e\left(s, g_{i}\right)=e\left(t, g_{i}\right)$ for $1 \leq i \leq n$. (T10) implies $\{C, \dot{\neg} C\} \cap \mathcal{L}(s) \neq \emptyset$ and $\{C, \dot{\neg} C\} \cap \mathcal{L}(t) \neq \emptyset$. If $\dot{\rightarrow} C \in \mathcal{L}(s)$, then the claim yields $s \in(\dot{\neg} C)^{\mathcal{I}}$ contradicting $s \in C^{\mathcal{I}}$. Thus we obtain $C \in \mathcal{L}(s)$ and, in a similar way, $C \in \mathcal{L}(t)$. Finally, (T14) implies that $s=t$, and thus $\mathcal{I}$ satisfies $\mathcal{K}$.

We now proceed to prove termination, soundness, and completeness of the tableau algorithm presented in Section 4.2, starting with termination. In the following, we use $|D, \mathcal{R}, \mathcal{K}|$ to denote $\left|\mathrm{cl}^{+}(D, \mathcal{R}, \mathcal{K})\right|$. Recall that this number is polynomial in the size of $D, \mathcal{R}, \mathcal{K}$.

Lemma C.2 (Termination). Let $\mathcal{D}$ be a key-admissible concrete domain. When started with a $\mathcal{S H O} \mathcal{Q K}(\mathcal{D})$ concept $D$ in $N N F$, a role box $\mathcal{R}$, and a path-free key box $\mathcal{K}$ in $N N F$, the tableau algorithm terminates.

Proof. Assume that there are $D, \mathcal{R}$, and $\mathcal{K}$ such that the tableau algorithm does not terminate. Since $\mathcal{D}$ is key-admissible, this means that there is an infinite sequence $S_{0}, S_{1}, \ldots$ of completion systems such that (a) $S_{0}$ is the initial completion system $S_{D}$ and (b) $S_{i+1}$ is the result of applying a completion rule to $S_{i}$.

This is only possible if the $\mathrm{R} \exists$ or the $R \geqslant$ rules are applied infinitely often: it is easily seen that the rules $\mathrm{R} \sqcap, \mathrm{R} \sqcup, \mathrm{R} \leqslant, \mathrm{R} \exists \mathrm{c}, \mathrm{R} \forall, \mathrm{R} \forall{ }_{+}$, Rch, and $\mathrm{R} \approx_{a}$ can only be applied finitely often to completion systems whose set of abstract nodes $V_{a}$ does not increase since they either add concepts into node labels (whose size is bounded), or they add concrete nodes (whose number is bounded linearly by the number of abstract nodes), or they remove abstract nodes from the forest. Hence there is a sub-sequence $S_{i_{1}}, S_{i_{2}}, \ldots$ of $S_{0}, S_{1}, \ldots$ such that $S_{i_{j}}$ is the result of applying the $\mathrm{R} \exists$ or the $\mathrm{R} \geqslant$ rule to $S_{i_{j}-1}$. Let $s_{i_{\ell}}$ be the abstract node to which the $\mathrm{R} \exists$ or the $\mathrm{R} \geqslant$ rule was applied in $S_{i_{\ell}-1}$. Since $s \prec t$ implies that $t$ was not generated before $s$, the linear ordering $\prec$ is well-founded. Thus, we find an infinite subsequence $S_{j_{1}}, S_{j_{2}}, \ldots$ of $S_{i_{1}}, S_{i_{2}}, \ldots$ such that either $s_{j_{\ell}}=s_{j_{\ell+1}}$ for each $\ell \geq 1$ or $s_{j_{\ell}} \prec s_{j_{\ell+1}}$ for each $\ell \geq 1$. The former, however, is not possible since the $\mathrm{R} \exists$ and the $\mathrm{R} \geqslant$ rules are applied at most once per node and concept in $\operatorname{cl}(D, \mathcal{R}, \mathcal{K})$ : even if a node is removed, the label copying performed by the $\mathrm{R} \leqslant$ rule together with clashes of type (C3) ensures that the $\mathrm{R} \geqslant$ rule is not re-applied to the same concept and node. Thus only the second option remains: there is a subsequence $S_{j_{1}}, S_{j_{2}}, \ldots$ of $S_{i_{1}}, S_{i_{2}}, \ldots$ such that $s_{j_{\ell}} \prec s_{j_{\ell+1}}$ for each $\ell \geq 1$. Let $\mathcal{L}_{j}$ be the labeling function in $S_{j}$. Since each abstract node is labeled with a subset $\mathcal{L}_{j}$ of $\mathrm{cl}^{+}(D, \mathcal{R}, \mathcal{K})$, there are nodes $s_{j_{k}} \prec s_{j_{\ell}}$ with $k \lesseqgtr \ell$ and $\mathcal{L}_{j_{k}}\left(s_{j_{k}}\right)=\mathcal{L}_{j_{\ell}}\left(s_{j_{\ell}}\right)$. Now node labels can only increase and, if a node $t$ is removed, its label is conjoined to the label of a node $\hat{t}$ with $\hat{t} \prec t$. Thus there is a node $t$ in the completion system $S_{j_{\ell}}$ with $t \prec s_{j_{\ell}}$ and $\mathcal{L}_{j_{\ell}}\left(s_{j_{\ell}}\right) \subseteq \mathcal{L}_{j_{\ell}}(t)$. By definition, $s_{j_{\ell}}$ is thus blocked in $S_{j_{\ell}}$, contradicting the assumption that the $\mathrm{R} \exists$ or the $\mathrm{R} \geqslant$ rule is applied to $s_{j_{\ell}}$ in $S_{j_{\ell}}$.

Lemma C.3 (Soundness). If the expansion rules can be applied to a $\mathcal{S H O Q \mathcal { K }}(\mathcal{D})$ concept $D$ in NNF, a role box $\mathcal{R}$, and a path-free key box $\mathcal{K}$ such that they yield a complete and clash-free completion forest, then $D$ has a tableau w.r.t. $\mathcal{R}$ and $\mathcal{K}$. 
Proof. Let $S=\left(\left(V_{a}, V_{c}, E, \mathcal{L}\right), \mathcal{P}, \sim_{c}, \prec\right)$ be a complete and clash-free completion system. We can find a solution $\delta$ for $\zeta_{S}$ such that $\delta(x)=\delta(y)$ iff $x \sim_{c} y$ : only the R $\exists$ c rule updates the predicate conjunction $\zeta_{S}$, and after each rule application the $\sim_{c}$ relation is updated using the concrete equivalence that a $\mathcal{D}$-tester returns for $\zeta_{S}$ (note that $\zeta_{S}$ is satisfiable due to clash-freeness). According to Definition 4.1, we can thus find a solution $\delta$ as required. From $S$ and $\delta$, we define a finite tableau $T=\left(\mathbf{S}_{a}, \mathbf{S}_{c}, \hat{E}, \hat{\mathcal{L}}, \hat{\mathcal{P}}\right)$ as follows:

$$
\begin{aligned}
\mathbf{S}_{a} & :=\left\{s \in V_{a} \mid s \text { occurs in } S \text { and is not blocked }\right\} \\
\mathbf{S}_{c} & :=\left\{\delta(x) \mid(s, x) \in E(g) \text { for some } s \in \mathbf{S}_{a} \text { and some } g\right\} \\
\hat{\mathcal{L}}(s) & \left.:=\mathcal{L}(s) \cap \mathrm{cl}(D, \mathcal{R}, \mathcal{K}) \text { (the intersection is due to the auxiliary concepts } A_{i}^{n R C}\right), \\
\hat{E}(s, t) & :=\left\{R \mid t \text { is an } R \text {-successor of } s \text { or } t \text { blocks an } R \text {-successor } t^{\prime} \text { of } s\right\} \\
e(s, g) & := \begin{cases}\delta(x) & \text { if } x \text { is a } g \text {-successor of } s \\
\text { undefined } & \text { if } x \text { has no } g \text {-successor }\end{cases} \\
\hat{\mathcal{P}} & :=\text { the restriction of } \mathcal{P} \text { to } \mathbf{S}_{c} .
\end{aligned}
$$

Note that the function $e$ is well-defined due to the definition of adding g-successors. It remains to show that $T$ satisfies (T1)-(T14), which is basically a consequence of $S$ being clash-free and complete.

- (T1) is satisfied since $S$ does not contain a clash (C1).

- (T2) is satisfied since the $\mathrm{R} \sqcap$ rule cannot be applied, and thus $C_{1} \sqcap C_{2} \in \hat{\mathcal{L}}(s)$ implies $C_{1}, C_{2} \in \hat{\mathcal{L}}(s)$.

- (T3) is satisfied since the R $\sqcup$ rule cannot be applied, and thus $C_{1} \sqcup C_{2} \in \hat{\mathcal{L}}(s)$ implies $\left\{C_{1}, C_{2}\right\} \cap \hat{\mathcal{L}}(s) \neq \emptyset$.

- For (T4), consider $s, t \in \mathbf{S}_{a}$ with $R \in \hat{E}(s, t)$ and $R \stackrel{\Xi}{=} R^{\prime}$. Then $R \in \hat{E}(s, t)$ implies that $t$ is or blocks an $R$-successor of $s$. By definition of "successor", $t$ is or blocks an $R^{\prime}$-successor of $s$, and thus $R^{\prime} \in \hat{E}(s, t)$.

- For (T5), let $\forall R . C \in \hat{\mathcal{L}}(s)$ and $R \in \hat{E}(s, t)$. If $t$ is an $R$-successor of $s$, then $s$ not being blocked implies $C \in \mathcal{L}(t)$ since the $\mathrm{R} \forall$ rule cannot be applied. If $t$ blocks an $R$-successor $t^{\prime}$ of $s$, then $s$ not being blocked and the fact that the $\mathrm{R} \forall$ rule cannot be applied yields $C \in \mathcal{L}\left(t^{\prime}\right)$, and the blocking condition implies $C \in \mathcal{L}(t)$.

In both cases, we thus have $C \in \hat{\mathcal{L}}(t)$.

- (T6) and (T7) are satisfied for the same reasons as (T5) with $\mathrm{R} \forall$ replaced with $\mathrm{R} \exists$ and $\mathrm{R} \forall_{+}$.

- For (T8), consider $s$ with $(\geqslant n R C) \in \hat{\mathcal{L}}(s)$. Hence $(\geqslant n R C) \in \mathcal{L}(s)$ and completeness of $S$ implies the existence of $R$-successors $t_{1}, \ldots, t_{n}$ of $s$ with $C \in \mathcal{L}\left(t_{i}\right)$ and $t_{i} \neq t_{j}$ for $i \neq j$. The latter implies, for each $i \neq j$, the existence of integers $k, \ell$ such that $k \neq \ell, A_{k}^{n R C} \in \mathcal{L}\left(t_{i}\right)$, and $A_{\ell}^{n R C} \in \mathcal{L}\left(t_{j}\right)$. For (T8) to be satisfied, it remains to verify that

- no $t_{i}$ can block a $t_{j}$ : if this was the case, the blocking condition would imply that $\left\{A_{k}^{n R C}, A_{\ell}^{n R C}\right\} \subseteq \mathcal{L}\left(t_{i}\right)$. 
- no $t$ can block both $t_{i}$ and $t_{j}$ with $i \neq j$ : similarly, this would imply that $\left\{A_{k}^{n R C}, A_{\ell}^{n R C}\right\} \subseteq \mathcal{L}(t)$.

In each case, we would have a clash (C3), in contradiction to $S$ being clash-free.

- For (T9), consider $s$ with $(\leqslant n R C) \in \hat{\mathcal{L}}(s)$. Hence $(\leqslant n R C) \in \mathcal{L}(s)$ and, since the $\mathrm{R} \leqslant$ rule cannot be applied, there are at most $n R$-successors $t_{i}$ of $s$. Since each $t_{i}$ is either not blocked or blocked by exactly one other node (due to $\prec$ being a linear ordering), there are at most $n u_{i} \in \mathbf{S}_{a}$ with $R \in \hat{E}\left(s, u_{i}\right)$ and $C \in \hat{\mathcal{L}}\left(u_{i}\right)$.

- For (T10), let $(\leqslant n R C) \in \hat{\mathcal{L}}(s)$ and $R \in \hat{E}(s, t)$. Hence $(\leqslant n R C) \in \mathcal{L}(s)$ and $t$ either is an $R$-successor of $s$ or blocks an $R$-successor of $s$. In the first case, nonapplicability of the Rch rule implies that $\{C, \dot{\neg} C\} \cap \mathcal{L}(t) \neq \emptyset$. In the second case, $\{C, \dot{\neg} C\} \cap \mathcal{L}\left(t^{\prime}\right) \neq \emptyset$ for $t^{\prime}$ the $R$-successor of $s$ blocked by $t$, and thus the blocking condition yields $\{C, \dot{\neg} C\} \cap \mathcal{L}(t) \neq \emptyset$. In both cases, this implies $\{C, \dot{\neg} C\} \cap \hat{\mathcal{L}}(t) \neq \emptyset$.

Next, consider $\left(g_{1}, \ldots, g_{n}\right.$ keyfor $\left.C\right) \in \mathcal{K}$ and $s$ such that $e\left(s, g_{i}\right)$ is defined for each $i$. Hence $s$ has a $g_{i}$-successor for each $i$, and thus $s$ not being blocked and the nonapplicability of the Rch rule imply that $\{C, \dot{\neg} C\} \cap \hat{\mathcal{L}}(t) \neq \emptyset$.

- For (T11), consider $N \in \hat{\mathcal{L}}(s) \cap \hat{\mathcal{L}}(t)$. By definition, $N \in \mathcal{L}(s) \cap \mathcal{L}(t)$ and thus $s \approx_{a} t$. Moreover, totality of $\prec$ implies that we can assume without loss of generality that $s \prec t$ or $s=t$. Thus non-applicability of the $\mathrm{R} \approx_{a}$ rule implies that $\mathcal{L}(t) \subseteq \mathcal{L}(s)$, and thus $t$ not being blocked implies $s=t$.

- (T12) is satisfied since the rule $\mathrm{R} \exists$ c cannot be applied.

- For (T13), clash-freeness implies the satisfiability of

$$
\bigwedge_{P \text { used in } D, \mathcal{K}} \bigwedge_{\left(x_{1}, \ldots, x_{n}\right) \in \mathcal{P}(P)} P\left(x_{1}, \ldots, x_{n}\right) .
$$

By choice of $\delta, \delta(x)=\delta(y)$ iff $x \sim_{c} y$, and thus $(\mathbf{T 1 3})$ is satisfied.

- For (T14), let $\left(g_{1}, \ldots, g_{n}\right.$ keyfor $\left.C\right) \in \mathcal{K}, C \in \hat{\mathcal{L}}(s) \cap \hat{\mathcal{L}}(t)$, and $e\left(s, g_{i}\right)=e\left(t, g_{i}\right)$, for all $1 \leq i \leq n$. Thus $C \in \mathcal{L}(s) \cap \mathcal{L}(t)$ and, by choice of $e$ and $\delta$, we have $x_{i} \sim_{c} y_{i}$ for $g_{i} \in E\left(s, x_{i}\right) \cap E\left(t, y_{i}\right)$. Hence $s \approx_{a} t$. Without loss of generality, we assume that $s \prec t$ or $s=t$. Thus non-applicability of the $\mathrm{R} \approx_{a}$ rule implies that $\mathcal{L}(t) \subseteq \mathcal{L}(s)$, and thus $t$ not being blocked implies $s=t$.

- (T15) is satisfied by definition of $T$ and since $S$ does not contain a clash (C4).

Lemma C.4 (Completeness). If a $\mathcal{S H O} \mathcal{Q K}(\mathcal{D})$-concept $D$ in NNF has a tableau w.r.t. a role box $\mathcal{R}$ and a path-free key box $\mathcal{K}$, then the expansion rules can be applied to $D, \mathcal{R}$, and $\mathcal{K}$ such that they yield a complete and clash-free completion forest. 
Proof. Given a tableau $T=\left(\mathbf{S}_{a}, \mathbf{S}_{c}, \hat{\mathcal{L}}, \hat{E}, e, \hat{\mathcal{P}}\right)$ for $D$ w.r.t. $\mathcal{R}$ and $\mathcal{K}$, we can "guide" the non-deterministic rules $\mathrm{R} \sqcup, \mathrm{Rch}$, and $\mathrm{R} \approx_{\mathrm{a}}$ in such a way that each rule application preserves clash-freeness. This together with termination from Lemma C.2 finishes the proof.

Along with rule application, we perform a stepwise construction of a total mapping $\pi$ that takes abstract nodes of the completion forest to elements of $\mathbf{S}_{a}$ and concrete nodes of the completion forest to elements of $\mathbf{S}_{c}$.

- $\mathcal{L}(s) \cap \mathrm{cl}(D, \mathcal{R}, \mathcal{K}) \subseteq \hat{\mathcal{L}}(\pi(s))$ for each $s \in V_{a}$,

- if $t$ is an $R$-successor of $s$, then $R \in \hat{E}(\pi(s), \pi(t))$,

- if $x$ is a $g$-successor of $s$, then $e(\pi(s), g)=\pi(x)$,

- $x \sim_{\mathrm{c}} y$ iff $\pi(x)=\pi(y)$, and

- if $s \neq t$, then $\pi(s) \neq \pi(t)$.

A mapping satisfying these four conditions is called correct in the following. Note that a completion system for which there exists a correct mapping does not contain a clash: due to (T1) and the first property, we do not encounter a clash (C1). A clash (C3) cannot occur due to the last property. The first and the third property together with (T15) ensure that a clash (C4) does not occur. Finally, a clash (C2) cannot occur for the following reason: by construction of $\mathcal{P}$ and since edges labelled with abstract features are never removed, for each tuple $\left(x_{1}, \ldots, x_{n}\right) \in \mathcal{P}(P)$, we find an abstract node $s$ and paths $u_{1}, \ldots, u_{n}$ such that $\exists u_{1}, \ldots, u_{n} . P \in \mathcal{L}(s)$ and $x_{i}$ is a $u_{i}$-successor of $s$ for $1 \leq i \leq n$. Thus, the first, second, and third property together with (T12) and (T13) ensure that the conjunction

$$
\bigwedge_{\substack{P \text { used in } D, \mathcal{K} \\\left(x_{1}, \ldots, x_{n}\right) \in \mathcal{P}(P)}} P\left(\pi\left(x_{1}\right), \ldots, \pi\left(x_{n}\right)\right)
$$

has a solution $\delta$ with $\delta(\pi(x)) \neq \delta(\pi(x))$ iff $\pi(x) \neq \pi(y)$. By the fourth property, setting $\delta^{\prime}(x):=\delta(\pi(x))$ for all $x \in V_{c}$ thus yields a solution $\delta^{\prime}$ for $\zeta_{S}$.

The total mapping $\pi$ is inductively defined as follows: let $\delta$ be a solution for the equation in (T13). Choose a node $\hat{s}_{0}$ with $D \in \hat{\mathcal{L}}\left(\hat{s}_{0}\right)$, and set $\pi\left(s_{0}\right):=\hat{s}_{0}$ for $s_{0}$ the (only) node of the initial completion forest. Obviously, $\pi$ is correct. We will now show that each completion rule can be applied in such a way that $\pi$ either is still correct or that $\pi$ can be extended to a correct mapping.

- An application of the rule $\mathrm{R} \sqcap$ preserves correctness of $\pi$ due to (T2).

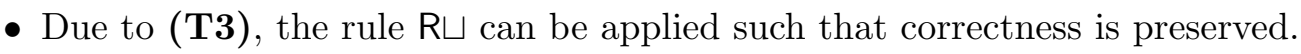

- If the rule $\mathrm{R} \exists$ adds a new node $t$ for $\exists R . C \in \mathcal{L}(s)$, then correctness implies $\exists R . C \in$ $\hat{\mathcal{L}}(\pi(s))$, and thus (T6) implies the existence of some $\hat{t} \in \mathbf{S}_{a}$ with $R \in E(\pi(s), \hat{t})$ and $C \in \hat{\mathcal{L}}(\hat{t})$. Thus extending $\pi$ with $\pi(t):=\hat{t}$ obviously yields a correct mapping.

- If the rule $\mathrm{R} \geqslant$ adds $n$ nodes $t_{i}$ for $(\geqslant n R C) \in \mathcal{L}(s)$, then correctness implies $(\geqslant n R C) \in \hat{\mathcal{L}}(\pi(s))$, and thus (T8) implies the existence of $\hat{t}_{1}, \ldots, \hat{t}_{n} \in \hat{\mathbf{S}}_{a}$ with $\hat{t}_{i} \neq \hat{t}_{j}$ for $i \neq j, R \in E\left(\pi(s), \hat{t}_{i}\right)$, and $C \in \hat{\mathcal{L}}\left(\hat{t}_{i}\right)$. Thus extending $\pi$ with $\pi\left(t_{i}\right):=\hat{t}_{i}$ obviously yields a correct mapping. 
- Assume that the $\mathrm{R} \leqslant$ rule is applicable to a node $s$ with $(\leqslant n R C) \in \mathcal{L}(s)$ and more than $n R$-successors $t_{i}$ with $C \in \mathcal{L}\left(t_{i}\right)$. Then correctness implies that $(\leqslant n R C) \in$ $\hat{\mathcal{L}}(\pi(s)), R \in \hat{E}\left(\pi(s), \pi\left(t_{i}\right)\right)$, and $C \in \hat{\mathcal{L}}\left(t_{i}\right)$. Thus, by (T9), there are $i \neq j$ with $\pi\left(t_{i}\right)=\pi\left(t_{j}\right)$. Again, correctness implies that not $t_{i} \neq t_{j}$ and, without loss of generality, we can assume that $t_{i} \prec t_{j}$. Hence applying the rule and thereby merging $\mathcal{L}\left(t_{j}\right)$ into $\mathcal{L}\left(t_{i}\right)$ preserves correctness.

- For the rule $\mathrm{R} \exists \mathrm{c}, \pi$ can be extended in a similar way as for $\mathrm{R} \exists$ : if a new $g_{i}$-successor $x_{i}$ of $s$ is added, then extending $\pi$ with $\pi\left(x_{i}\right):=e\left(\pi(s), g_{i}\right)$ yields a correct $\pi$. Moreover, (T13) ensures that $\sim_{c}$ can be updated in such a way that the fourth condition is preserved.

- For the $\mathrm{R} \forall$ rule, $\pi$ does not need to be extended, and (T5), (T4), and the definition of $R$-successors imply that correctness is preserved.

- The $\mathrm{R} \forall+$ rule is similar, with the only difference that (T7) takes the place of (T5).

- Due to (T10), the rule Rch can be applied without violating correctness.

- For $\mathrm{R} \approx_{\mathrm{a}}$, we consider two reasons for $\mathrm{R} \approx_{\mathrm{a}}$ to be applicable:

- $N \in \mathcal{L}(s) \cap \mathcal{L}(t)$. Then correctness of $\pi$ and (T11) imply that $\pi(s)=\pi(t)$.

- $\left(g_{1}, \ldots, g_{n}\right.$ keyfor $\left.C\right) \in \mathcal{K}, C \in \mathcal{L}(s) \cap \mathcal{L}(t)$, and $g_{i} \in E\left(s, x_{i}\right) \cap E\left(t, y_{i}\right)$ and $x_{i} \sim_{\mathrm{c}} y_{i}$ for $1 \leq i \leq n$. Then correctness implies that $\hat{e}\left(\pi(s), g_{i}\right)=e\left(\pi(t), g_{i}\right)$, and thus (T14) together with the first property of correctness imply that $\pi(s)=\pi(t)$.

In both cases, applying $\mathrm{R} \approx_{\mathrm{a}}$ to $s$ and $t$ preserves correctness.

As an immediate consequence of Lemmas 4.2, C.2, C.3, and C.4, the tableau algorithm always terminates and answers " $D$ is satisfiable w.r.t. $\mathcal{R}$ and $\mathcal{K}$ " if and only if the input concept $D$ is satisfiable w.r.t. the input role box $\mathcal{R}$ and the input key box $\mathcal{K}$. Since concept satisfiability w.r.t. TBoxes can be reduced to concept satisfiability without TBoxes, we obtain the following result:

Proposition C.5. Let $\mathcal{D}$ be a key-admissible concrete domain. The tableau algorithm decides satisfiability of $\mathcal{S H O Q K}(\mathcal{D})$ concepts w.r.t. TBoxes, role boxes, and path-free key boxes.

It is not hard to verify that the proof of Lemma C. 4 together with Lemmas 4.2 and C.2 yield a bounded model property for $\mathcal{S H O} \mathcal{Q K}(\mathcal{D})$, where the bound is exponential.

Corollary C.6. If a $\mathcal{S H O Q \mathcal { K }}(\mathcal{D})$-concept $D$ is satisfiable w.r.t. a role box $\mathcal{R}$ and a pathfree key box $\mathcal{K}$, then $D$ is satisfiable w.r.t. $\mathcal{R}$ and $\mathcal{K}$ in a model of size at most $\left|\Delta_{\mathcal{I}}\right| \leq 2^{m}$ for $m=\# \mathrm{cl}^{+}(D, \mathcal{R}, \mathcal{K})$. 
Proof. If a $\mathcal{S H O} \mathcal{H} \mathcal{K}(\mathcal{D})$-concept $D$ is satisfiable w.r.t. a role box $\mathcal{R}$ and a path-free key box $\mathcal{K}$, Lemma C.4 implies that the tableau algorithm constructs a complete and clash-free completion forest for $D, \mathcal{R}$, and $\mathcal{K}$. By the definition of blocking, the number of abstract nodes in a completion forest that are not blocked is bounded by $2^{m}$ : if $s \neq t \in V_{a}$ are abstract nodes in a completion forest and $\mathcal{L}(s)=\mathcal{L}(t)$, then either $s$ blocks $t, t$ blocks $s$, or they are both blocked by another node $u$. Moreover, it is easily seen that the number of concrete successors per abstract node is bounded by the number of concrete features in $C, \mathcal{R}$, and $\mathcal{K}$. Now, in the proof of Lemma C.4, the abstract nodes in the tableau constructed from a complete and clash-free completion forest coincide with the nodes that are not blocked in the completion forest. Finally, in the proof of Lemma 4.2 the interpretation domain of a model constructed from a tableau coincides with the abstract nodes in the tableau. Summing up, a $\mathcal{S H O} \mathcal{Q K}(\mathcal{D})$-concept that is satisfiable w.r.t. $\mathcal{R}$ and $\mathcal{K}$ has a model of size $\left|\Delta_{\mathcal{I}}\right| \leq 2^{m}$.

\section{References}

Areces, C., Blackburn, P., \& Marx, M. (1999). A road-map on complexity for hybrid logics. In Flum, J., \& Rodríguez-Artalejo, M. (Eds.), Computer Science Logic, No. 1683 in Lecture Notes in Computer Science, pp. 307-321. Springer-Verlag.

Baader, F., Horrocks, I., \& Sattler, U. (2002a). Description logics for the semantic web. KI - Künstliche Intelligenz, 16(4), 57-59.

Baader, F., Lutz, C., Sturm, H., \& Wolter, F. (2002b). Fusions of description logics and abstract description systems. Journal of Artificial Intelligence Research (JAIR), 16, $1-58$.

Baader, F., \& Sattler, U. (1998). Description logics with concrete domains and aggregation. In Prade, H. (Ed.), Proceedings of the 13th European Conference on Artificial Intelligence (ECAI'98), pp. 336-340. John Wiley \& Sons.

Baader, F., Calvanese, D., McGuinness, D. L., Nardi, D., \& Patel-Schneider, P. F. (2003). The Description Logic Handbook: Theory, implementation and applications. Cambridge University Press, Cambridge, MA, USA.

Baader, F., \& Hanschke, P. (1991a). A scheme for integrating concrete domains into concept languages. In Proceedings of the 12th International Joint Conference on Artificial Intelligence (IJCAI-91), pp. 452-457, Sydney, Australia.

Baader, F., \& Hanschke, P. (1991b). A scheme for integrating concrete domains into concept languages. DFKI research report RR-91-10, German Research Center for Artificial Intelligence (DFKI).

Baader, F., \& Hanschke, P. (1992). Extensions of concept languages for a mechanical engineering application. In Proceedings of the 16th German AI-Conference (GWAI92), Vol. 671 of Lecture Notes in Computer Science, pp. 132-143. Springer-Verlag.

Baader, F., \& Sattler, U. (2000). Tableau algorithms for description logics. In Dyckhoff, R. (Ed.), Proceedings of the International Conference on Automated Reasoning with Tableaux and Related Methods (Tableaux 2000), Vol. 1847 of Lecture Notes in Artificial Intelligence, pp. 1-18. Springer-Verlag. 
Berger, R. (1966). The undecidability of the domino problem. Memoirs of the American Mathematical Society, 66, 1-72.

Berners-Lee, T., Hendler, J., \& Lassila, O. (2001). The semantic web. Scientific American, $284(5), 34-43$.

Börger, E., Grädel, E., \& Gurevich, Y. (1997). The Classical Decision Problem. Perspectives in Mathematical Logic. Springer-Verlag.

Borgida, A., \& Patel-Schneider, P. F. (1994). A semantics and complete algorithm for subsumption in the CLASSIC description logic. Journal of Artificial Intelligence Research, 1, 277-308.

Borgida, A., \& Weddell, G. E. (1997). Adding uniqueness constraints to description logics (preliminary report). In Bry, F., Ramakrishnan, R., \& Ramamohanarao, K. (Eds.), Proceedings of the 5th International Conference on Deductive and Object-Oriented Databases (DOOD97), Vol. 1341 of LNCS, pp. 85-102. Springer.

Calvanese, D., De Giacomo, G., \& Lenzerini, M. (1998). On the decidability of query containment under constraints. In Proceedings of the 17th ACM SIGACT-SIGMODSIGART Symposium on Principles of Database Systems (PODS'98), pp. 149-158.

Calvanese, D., De Giacomo, G., \& Lenzerini, M. (2000). Keys for free in description logics. In Baader, F., \& Sattler, U. (Eds.), Proceedings of the 2000 International Workshop in Description Logics (DL2000), No. 33 in CEUR-WS (http://ceur-ws.org/), pp. 79-88.

Calvanese, D., Lenzerini, M., \& Nardi, D. (1998). Description logics for conceptual data modeling. In Chomicki, J., \& Saake, G. (Eds.), Logics for Databases and Information Systems, pp. 229-263. Kluwer Academic Publisher.

Dean, M., Connolly, D., van Harmelen, F., Hendler, J., Horrocks, I., McGuinness, D. L., Patel-Schneider, P. F., \& Stein, L. A. (2002). Web ontology language (OWL) reference version 1.0. W3C Working Draft.

Fensel, D., van Harmelen, F., Horrocks, I., McGuinness, D. L., \& Patel-Schneider, P. F. (2001). OIL: An ontology infrastructure for the semantic web. IEEE Intelligent Systems, 16(2), 38-45.

Graham, R. L., Knuth, D. E., \& Patashnik, O. (1990). Concrete Mathematics. Addison Wesley Publ. Co., Reading, Massachussetts.

Haarslev, V., Lutz, C., \& Möller, R. (1998). Foundations of spatioterminological reasoning with description logics. In Cohn, A., Schubert, L., \& S.C.Shapiro (Eds.), Proceedings of the 6th International Conference on Principles of Knowledge Representation and Reasoning (KR'98), pp. 112-124. Morgan Kaufman.

Haarslev, V., \& Möller, R. (2001). RACER system description. In Goré, R., Leitsch, A., \& Nipkow, T. (Eds.), Proceedings of the 1st International Joint Conference on Automated Reasoning (IJCAR'01), No. 2083 in Lecture Notes in Artificial Intelligence, pp. 701-705. Springer-Verlag.

Haarslev, V., Möller, R., \& Wessel, M. (2001). The description logic $\mathcal{A} \mathcal{L C N} \mathcal{H}_{R^{+}}$extended with concrete domains: A practically motivated approach. In Goré, R., Leitsch, A., 
\& Nipkow, T. (Eds.), Proceedings of the 1st International Joint Conference on Automated Reasoning IJCAR'01, No. 2083 in Lecture Notes in Artificial Intelligence, pp. 29-44. Springer-Verlag.

Halpern, J. Y., \& Moses, Y. (1992). A guide to completeness and complexity for modal logics of knowledge and belief. Artificial Intelligence, 54 (3), 319-380.

Hollunder, B., \& Baader, F. (1991). Qualifying number restrictions in concept languages. In Proceedings of the 2nd International Conference on Principles of Knowledge Representation and Reasoning (KR'91), pp. 335-346, Boston, MA, USA.

Hopcroft, J. E., \& Ullman, J. D. (1979). Introduction to Automata Theory, Languages and Computation. Addison-Wesley.

Horrocks, I., Sattler, U., \& Tobies, S. (2000). Practical reasoning for very expressive description logics. Logic Journal of the IGPL, 8(3), 239-264.

Horrocks, I. (1998). Using an expressive description logic: FaCT or fiction?. In Proceedings of the 6th International Conference on the Principles of Knowledge Representation and Reasoning (KR98), pp. 636-647.

Horrocks, I. (2002). Reasoning with expressive description logics: Theory and practice. In Voronkov, A. (Ed.), Proceedings of the 18th International Conference on Automated Deduction (CADE 2002), No. 2392 in Lecture Notes in Artificial Intelligence, pp. 1-15. Springer.

Horrocks, I., Patel-Schneider, P. F., \& van Harmelen, F. (2002). Reviewing the design of DAML+OIL: An ontology language for the semantic web. In Proceedings of the 18th National Conference on Artificial Intelligence (AAAI 2002), pp. 792-797.

Horrocks, I., \& Sattler, U. (2001). Ontology reasoning in the $\mathcal{S H O Q}(D)$ description logic. In Nebel, B. (Ed.), Proceedings of the 17th International Joint Conference on Artificial Intelligence (IJCAI'01), pp. 199-204. Morgan-Kaufmann.

Horrocks, I., Sattler, U., \& Tobies, S. (1999). Practical reasoning for expressive description logics. In Ganzinger, H., McAllester, D., \& Voronkov, A. (Eds.), Proceedings of the 6th International Conference on Logic for Programming and Automated Reasoning (LPAR'99), No. 1705 in Lecture Notes in Artificial Intelligence, pp. 161-180. SpringerVerlag.

Kamp, G., \& Wache, H. (1996). CTL - a description logic with expressive concrete domains. Tech. rep. LKI-M-96/01, Laboratory for Artificial Intelligence (LKI), Universitity of Hamburg, Germany.

Khizder, V. L., Toman, D., \& Weddell, G. E. (2001). On decidability and complexity of description logics with uniqueness constraints. In den Bussche, J. V., \& Vianu, V. (Eds.), Proceedings of the 8th International Conference on Database Theory (ICDT2001), Vol. 1973 of $L N C S$, pp. 54-67. Springer.

Knuth, D. (1968). The Art of Computer Programming, Vol. 1. Addison-Wesley.

Lutz, C. (2003). Description logics with concrete domains-a survey. In Advances in Modal Logics Volume 4, pp. 265-296. World Scientific Publishing Co. Pte. LTd. 
Lutz, C. (2002a). The Complexity of Reasoning with Concrete Domains. Ph.D. thesis, LuFG Theoretical Computer Science, RWTH Aachen, Germany.

Lutz, C. (2002b). PSPACE reasoning with the description logic $\mathcal{A} \mathcal{L C} \mathcal{F}(\mathcal{D})$. Logic Journal of the IGPL, 10(5), 535-568.

Lutz, C. (2002c). Reasoning about entity relationship diagrams with complex attribute dependencies. In Horrocks, I., \& Tessaris, S. (Eds.), Proceedings of the International Workshop in Description Logics 2002 (DL2002), No. 53 in CEUR-WS (http://ceurws.org/), pp. 185-194.

Lutz, C. (2004). NExpTime-complete description logics with concrete domains. ACM Transactions on Computational Logic, 5(4), 669-705.

Lutz, C., Areces, C., Horrocks, I., \& Sattler, U. (2002). Keys, nominals, and concrete domains. LTCS-report 02-04, Technical University Dresden. See http://lat.inf.tudresden.de/research/reports.html.

Lutz, C., Areces, C., Horrocks, I., \& Sattler, U. (2003). Keys, nominals, and concrete domains. In Proceedings of the 18th International Joint Conference on Artificial Intelligence (IJCAI'03), pp. 349-354. Morgan-Kaufmann Publishers.

Pan, J. Z., \& Horrocks, I. (2002). Reasoning in the $\mathcal{S H O Q}\left(\mathcal{D}_{n}\right)$ description logic. In Horrocks, I., \& Tessaris, S. (Eds.), Proceedings of the International Workshop in Description Logics 2002 (DL2002), No. 53 in CEUR-WS (http://ceur-ws.org/), pp. 53-62.

Post, E. M. (1946). A variant of a recursively unsolvable problem. Bulletin of the American Mathematical Society, 52, 264-268.

Schild, K. D. (1991). A correspondence theory for terminological logics: Preliminary report. In Mylopoulos, J., \& Reiter, R. (Eds.), Proceedings of the 12th International Joint Conference on Artificial Intelligence (IJCAI-91), pp. 466-471. Morgan Kaufmann.

Schmidt-Schauß, M., \& Smolka, G. (1991). Attributive concept descriptions with complements. Artificial Intelligence, 48(1), 1-26. 